\title{
Kronenstruktur und -dynamik von Rein- und Mischbeständen aus Buche, Fichte und Douglasie
}

\author{
Dissertation \\ zur Erlangung des Doktorgrades (Dr. forest) \\ der Forstwissenschaftlichen Fakultät \\ der Georg-August-Universität Göttingen \\ vorgelegt von \\ Johannes Sauer \\ geboren am 23.02.1991 in Göttingen
}

Göttingen, Juli 2021 
Gefördert von der Deutschen Forschungsgemeinschaft (DFG)

Projektnummer 316045089

1. Gutachter: Prof. Dr. Jürgen Nagel

2. Gutachter: Prof. Dr. Christian Ammer

Tag der mündlichen Prüfung: 29.09.2021 


\section{Inhaltsverzeichnis}

1 Einleitung 1

1.1 Hintergrund des Forschungsprojektes . . . . . . . . . . . . . 2

1.2 Kronenstruktur und Mischbestände . . . . . . . . . . . 6

1.2.1 Kronenbreite ................. 6

1.2 .2 Mischbestände . . . . . . . . . . . . . . 7

1.3 Ziel der Arbeit . . . . . . . . . . . . . . . 8

2 Methoden und Datengrundlage $\quad 11$

2.1 Ertragskundliche Aufnahmeverfahren . . . . . . . . . . . . 11

2.2 Aufnahme des Kronenprofils . . . . . . . . . . . . . . . . . 12

2.3 Korrektur der Fotos für fotogrammetrische Auswertungen . . . . . 14

2.4 Auswertung der Kronenbilder mit der Software ImageJ . . . . . . . 16

2.5 Versuchsflächen . . . . . . . . . . . . . . . . 18

2.5.1 Farbschema und Abkürzungen der Baumarten . . . . . . . . 18

2.5.2 Versuchsflächen der Nordwestdeutschen Forstlichen Versuchsanstalt . . . . . . . . . . . . . . . . 19

2.5.3 Versuchsflächen GRK 2300 . . . . . . . . . . 20

2.5.4 Quintett 1 - Riefensbeek .............. 23

2.5.5 Quintett 2- Dassel . . . . . . . . . . . . . 24

2.5.6 Quintett 3-Winnefeld . . . . . . . . . . . 26

2.5.7 Quintett 4 - Nienover ............... 27

2.5.8 Quintett 5 - Nienburg ................ 29

2.5.9 Quintett 6 - Unterlüß . . . . . . . . . . . . 30

2.5.10 Quintett 7 - Göhrde II . . . . . . . . . . . . . . 32

2.5.11 Quintett 8 -Göhrde I . . . . . . . . . . . 33

2.6 Überlegungen zur Konkurrentenauswahl - einzelbaumbasierte Mischungskonstellation .................... 34

2.7 Nachbarschaftsabhängige Kronenform . . . . . . . . . . . . . . 36 
2.8 Aufbau eines dreidimensionalen Waldbestandes . . . . . . . . . . 40

2.9 Voxel zur Darstellung von dreidimensionalen Objekten . . . . . . . 41

2.10 Volumen- und Oberflächenberechnung mit Voxeldaten . . . . . . . 43

2.11 Beleuchtete Kronenmantelfläche . . . . . . . . . . . . . . . 45

2.12 Überlegungen zur Bestandesauswertung mit Hilfe des Modells . . . 46

2.13 Verwendete Software R . . . . . . . . . . . . . . . 47

$\begin{array}{llr}3 & \text { Ergebnisse } & \mathbf{5 0}\end{array}$

3.1 Kronenmaße und Allometrien in Rein- und Mischbestand . . . . . 50

3.1.1 Zusammenhang von BHD und Kronenbreite . . . . . . . . 50

3.1.2 Höhe der maximalen Kronenbreite . . . . . . . . . . . . . . 51

3.1.3 Vergleich der horizontalen Kronenausdehnung . . . . . . . 54

3.1.4 Asymmetrie der Kronen . . . . . . . . . . . . . . . . 55

3.2 Kronenprofile in Rein- und Mischbstand . . . . . . . . . . . 60

3.2 .1 Kronenprofildaten . . . . . . . . . . . . . 60

3.2 .2 Modellansatz . . . . . . . . . . . . . . . . 61

3.2.3 Reaktionsmuster auf veränderte Nachbarschaft . . . . . . 68

3.2.4 Eingrenzung der Modellschätzung . . . . . . . . . . 73

3.3 Modelleffekte auf Bestandesebene . . . . . . . . . . . . . . . . 74

3.4 Vergleich geschätzter Kronenradien mit Kronenablotungen auf NW-

FVA Versuchsflächen . . . . . . . . . . . . . . . . . . . 84

4 Diskussion $\quad 86$

4.1 Beurteilung der Versuchsflächen und des Datenmaterials auf den Versuchsflächen . . . . . . . . . . . . . . . 86

4.2 Beurteilung des entwickelten Modellansatzes . . . . . . . . . . 88

4.3 Geschätzte maximale Kronenradien . . . . . . . . . . . . . . . . . 91

4.4 Einfluss der direkten Nachbarschaft auf die funktionalen Merkmale von Buche, Douglasie und Fichte . . . . . . . . . . . . . . . . . 93

4.5 Mischbestandseffekte . . . . . . . . . . . . . . . 96 
4.5.1 Reaktionsmuster der Baumkronen . . . . . . . . . . 96

4.5.2 Bestandeseffekte des Kronenmodells . . . . . . . . . . . . . 97

4.6 Waldbauliche Konsequenzen für die Behandlung von Buchen-Fichten und Buchen-Douglasien Mischbeständen . . . . . . . . . . . . 99

4.7 Ausblick . . . . . . . . . . . . . . . . . . . . 102 


\section{Abstract}

In this study, crown traits were examined in order to analyze their change depending on the neighborhood of individual trees and based on this, to compare the crown structure and use of space in pure and mixed stands. The crown window method was used and further developed for uncomplicated data acquisition in order to capture the crown shape with a tablet or smartphone. From this data the parameters of a Weibull function were estimated with the help of statistical models, which then represents the crown shape based on the neighborhood of a single tree. The results show that the differences between pure and mixed stands are mainly due to the adaptability or plasticity of the beech crowns. It was found that the beech crowns react to a mixture with the conifers spruce and Douglas-fir. When mixed, the beech has a wider crown than in the pure stand. The height of the maximum crown width in mixture with Douglas-fir is below the Douglas-fir crown and lower than in the pure beech stand. Furthermore, the beech crowns in the mixed stand have a more oval and asymmetrical shape while the conifers show no crown asymmetry in mixed conditions. Douglas fir, on the other hand, has a rounder crown shape in mixture with beech. In summary, it can be said that beech can spread the crown under the higher growing Douglas-fir and compensate for the lost crown space with a wider, but lower crown.

The examinations with the help of the crown model constructed here confirm this statement. The mixed stands of beech with both types of conifers had a higher light canopy surface area than pure stands, each with the same basal area. The beech was able to achieve a light crown surface area that was up to 1.6 times higher than in the pure stand, especially in a small mixture proportion of $25 \%$ beech / 75\% Douglas-fir, while Douglas-fir showed no change in any of the mixing variants. Spruce, on the other hand, when mixed with beech, had a slightly smaller crown surface area, the higher the beech proportion was. Overall, mixed stands with both conifers show a positive effect on the total canopy surface of the stand. 
The beech-Douglas-fir mixture has a positive effect on the total crown volume, while the beech-spruce mixture shows no effect on the total crown volume because the larger beech crowns compensate for the smaller spruce crowns. A regression analysis has also shown that crown radii can be determined more precisely with the here constructed crown model for Douglas-fir, spruces, and beech in comparison to a symmetrical crown model or a crown-width-function.

\section{Einleitung}

Die hier vorliegende Arbeit ist Teil des Graduiertenkollegs „Enrichment of European beech forests with conifers: impacts of functional traits on ecosystem functioning", welches an der Georg-August-Universität Göttingen in Zusammenarbeit mit der Nordwestdeutschen Forstlichen Versuchsanstalt (NW-FVA) stattfindet. Das Graduiertenkolleg besteht aus elf Teilprojekten mit insgesamt 40 Versuchsflächen. Es sollen sowohl die wichtigsten funktionalen Merkmale der Baumarten und jene assoziierter Organismengruppen untersucht, als auch die Mechanismen, die diese mit den Ökosystemfunktionen verbinden (Enrico, 2021). Vor dem Hintergrund steigender Anforderungen an Ökosystemgüter und - dienstleistungen, welche Wälder erfüllen sollen, sind Mischwaldbestände von besonderem Interesse. Es wird allgemein angenommen, dass das Vorhandensein, die Häufigkeit, die Verteilung und die Vielfalt der funktionalen Merkmale für das Funktionieren des Ökosystems wichtiger sind als die Artenvielfalt an sich (Bengtsson, 1998; Nadrowski et al., 2010). Um diese Annahme genauer zu untersuchen, zielt das Forschungsprojekt darauf $\mathrm{ab}$, die funktionalen Merkmale und die damit verbundenen Mechanismen in Rein- und Mischbeständen mit den drei folgenden Baumarten zu identifizieren: die europäische Buche (FAGUS SYLVATICA L.), eine häufig anzutreffende und heimische Laubbaumart, die Fichte (PICEA ABIES (L.) KARST), eine einheimische Nadelbaumart, die hauptsächlich außerhalb ihres natürlichen Verbreitungsgebiets kultiviert wird, sowie die Douglasie (Pseudotsuga menziesil (mirb.) Fran$\mathrm{CO}$ ), eine nicht einheimische Nadelbaumart, welche aus dem pazifischen Nordwes- 
ten Nordamerikas stammt (Enrico, 2021). Es wird vermutet, dass Mischungen aus Buche mit Nadelbäumen eine höhere funktionale Vielfallt aufweisen als reine $\mathrm{Bu}-$ chenbestände und dass die Auswirkungen von zugemischten Nadelbäumen auf die Funktionen des Ökosystems bei Mischungen mit Douglasie stärker ausgeprägt sind (Thurm et al., 2016; Lu et al., 2016; Pretzsch et al., 2016; Mölder und Leuschner, 2014; Schuldt und Scherer-Lorenzen, 2014; Enrico, 2021).

\subsection{Hintergrund des Forschungsprojektes}

Die Auswirkungen von Baumartenmischungen auf die Funktionsweise von Ökosystemen sind noch nicht vollständig erforscht und können in räumlicher und zeitlicher Form variieren (Forrester, 2014). Anstelle der Artenvielfalt wird zunehmend die Vielfalt von funktionalen Merkmalen als wichtiger Indikator für die Bereitstellung von Ökosystemfunktionen gesehen (Díaz et al., 2006; Nadrowski et al., 2010; Lu et al., 2016; Bengtsson, 1998). Nach de Bello et al. (2010) haben funktionale Merkmale immer eine Verbindung zu der Funktion des Organismus. Sie fungieren als Anpassung an eine Veränderung der Umgebung. Das funktionale Merkmal bestimmt die Reaktion des Organismus auf Veränderungen (response trait) und seine Auswirkungen auf Ökosystemprozesse und Dienstleistungen (effect trait). Funktionale Merkmale werden als die wichtigsten ökologischen Attribute angesehen, durch die verschiedene Organismen und biologische Gemeinschaften die Ökosystemleistungen beeinflussen (Violle et al., 2007). In Waldökosystemen wurden Reaktionsund Wirkungsmerkmale (response/effect traits) jedoch selten gemeinsam untersucht, und es ist nur wenig über die Zusammenhänge bekannt, die die beobachteten Auswirkungen auf die Funktion des Ökosystems erklären können (Lavorel und Garnier, 2002; Lavorel, 2013). Im Allgemeinen wird angenommen, dass ein auf funktionalen Merkmalen basierender Ansatz zu realistischen Vorhersagen über die Folgen einer veränderten Artenvielfalt führt (Hillebrand und Matthiessen, 2009).

Dieser Ansatz wurde schon als Konzept für ähnliche Studien mit anderen Arten und Artenkombinationen und in anderen Regionen der Welt verwendet (Werner 
und Peacor, 2003; Garnier und Navas, 2012; Lavorel und Garnier, 2002). Eine nachhaltige Waldbewirtschaftung in Mitteleuropa beinhaltet produktive und stabile Wälder, die verschiedene Ökosystemgüter und -dienstleistungen bereitstellen (Nabuurs et al., 2007). Hierbei wird von einer Multifunktionalität des Waldes gesprochen (Röhrig et al., 2020). Die verschiedenen Funktionen sind unter anderem die Holzproduktion, biologische Vielfalt, ausgewogene Wasser- und Nährstoffflüsse, Kohlenstoffbindung, Landschaftsschutz und Erholung (Nabuurs et al., 2007; Bauhus et al., 2010; Röhrig et al., 2020). Die Unterschiede in der Bereitstellung dieser Multifunktionalität in verschiedenen Baumartenmischungen können mit dem auf funktionalen Merkmalen beruhenden Ansatz untersucht werden (Hillebrand und Matthiessen, 2009).

Mitteleuropa stellt eine Ausnahme und ein Beispiel für ein Waldökosystem dar, in dem ohne den Eingriff des Menschen Rotbuche im Reinbestand an vielen Standorten vorkommt, während in anderen Teilen der Welt häufig eine Mischung aus einer Vielzahl dort einheimischer Baumarten vorzufinden ist (Ellenberg und Leuschner, 2010; Schulze et al., 2010). In einer von (Hobi et al., 2015) durchgeführten Bestandsaufnahme des größten Buchenurwaldes in Europa wurde herausgefunden, dass die Buche dort die dominante Baumart ist. Obwohl Reinbestände auch eine Vielzahl von Ökosystemgütern und - dienstleistungen anbieten und die Forstwirtschaft in vielen Teilen der Welt auf diese angewiesen ist (Kanninen, 2010), gibt es einige Gründe, die gegen Reinbestände sprechen. Einige der Ökosystemfunktionen des Waldes können miteinander in Konflikt geraten, wie zum Beispiel eine hohe Holzproduktion mit Hilfe von Nadelbäumen und gleichzeitig die Bereitstellung einer guten Qualität des Sickerwassers (Schume et al., 2004). Darüber hinaus sind Monokulturen aus Gründen wie der Schädlingsanfälligkeit (Hu et al., 2009; Wermelinger, 2004) und Krankheiten einem hohen Risiko ausgesetzt. Aus diesen Gründen wurden in der Vergangenheit Reinbestände in Frage gestellt (Engelmark et al., 2001; Lamb et al., 2005; Verheyen et al., 2007). Neue Konzepte, einschließlich neuartiger Mischungsformen, sind erforderlich, um ein Gleichgewicht 
zwischen wirtschaftlichen, ökologischen und sozialen Zielen zu erreichen (Bauhus et al., 2010). Steigende Temperaturen, verringerte Wasserverfügbarkeit im Sommer und erhöhte Stickstoffeinträge könnten das Waldwachstum und die Baumartenzusammensetzung in vielen Teilen der Welt erheblich beeinflussen (IPCC, 2007). Wälder reagieren unterschiedlich auf sich ändernde klimatische Bedingungen, abhängig von örtlichen Standortmerkmalen, Artenzusammensetzung, Waldstruktur und waldbaulichen Behandlungen, die die Auswirkungen von Hitze, Dürre und Sturmereignissen verringern oder verstärken können. Eine der Hauptsorgen ist, dass Waldbestände durch sich schnell ändernde Umweltbedingungen bedroht sein könnten (Ammer, 2016). Daher wurde eine adaptive Waldbewirtschaftung vorgeschlagen, die darauf abzielt, die Funktionalität bewirtschafteter Wälder als Voraussetzung für die Erfüllung des zukünftigen Bedarfs an Waldökosystemdienstleistungen zu erhalten und zu entwickeln (Millar et al., 2007; Bolte et al., 2009; Pinkard et al., 2015; Ammer, 2016). Die Stressresistenz und Widerstandsfähigkeit von Waldökosystemen soll dadurch erhöht und deren Fähigkeit, dynamisch auf Auswirkungen des Klimawandels zu reagieren, unterstützt werden (Keenan, 2015; Millar et al., 2007; Ammer, 2016). Eine häufig diskutierte Option für widerstandsfähigere Wälder ist die Erhöhung des Anteils von Mischbeständen (Thurm et al., 2016; Metz et al., 2016; Lebourgeois et al., 2013). Diese Mischbestände können aus einheimischen und fremden Baumarten bestehen. Daten zu den Auswirkungen der Anreicherung einheimischer Bestände mit nicht einheimischen Baumarten sind selten (Lu et al., 2016; Hildebrandt und Knoke, 2009; Nichols et al., 2006; Senbeta et al., 2002), jedoch wird angenommen, dass dadurch die Waldökosysteme in ihrer Funktionalität erweitert werden (Oxbrough et al., 2016).

Im Gegensatz zu anderen Teilen der Welt hat die Forstwirtschaft in Mitteleuropa fremde Baumarten nur langsam eingeführt. Die Douglasie ist eine der wenigen Arten, die in größerem Maßstab seit Ende des 19. Jahrhunderts in Waldbeständen kultiviert werden (Reyer et al., 2010). Douglasien, welche aus nordamerikanischen Küstengebieten stammen (Pseudotsuga menziesii var. Menziesii), zeichnen sich 
durch höhere Erträge aus als einheimische Baumarten aus Europa und sind auch auf nährstoffarmen Standorten produktiver (Weller, 2011). Das Holz der Douglasie ist außerdem ein hochwertiges Nutzholz. Die Art kann sich durch Naturverjüngung regenerieren (Knoerzer, 1999) und es konnten bis jetzt keine schwerwiegenden negativen ökologischen Auswirkungen festgestellt werden (Schmid et al., 2014). Der erwartete Klimawandel hat viele Waldbesitzer dazu motiviert, sich in Zukunft auf diese Art zu verlassen, da davon ausgegangen wird, dass sie relativ trockentolerant ist (White, 1987; Vitali et al., 2017). Die Douglasie kann andere wirtschaftlich wichtige Nadelbäume wie die Fichte teilweise ersetzen, welche voraussichtlich von Sturmereignissen und insbesondere von einem Anstieg der Borkenkäferpopulationen infolge des projizierten Temperaturanstiegs und der Dürrehäufigkeit betroffen sein wird (Spellmann et al., 2007; Roloff und Grundmann, 2008; Lüpke, 2009; Kölling et al., 2009). Douglasie wird in Deutschland traditionell hauptsächlich in Reinbeständen angebaut und andernfalls häufig mit Rotbuche gemischt, einer sehr konkurrenzstarken, schattentoleranten einheimischen Art, die unter natürlichen Bedingungen in weiten Teilen Mitteleuropas dominant sein würde (Ellenberg und Leuschner, 2010; Schulze et al., 2010). Europäische Buchen-Douglasien Bestände bieten somit die Gelegenheit, die Mischung einer hochproduktiven nicht einheimischen Baumart mit einer einheimischen Baumart zu untersuchen. Dieser Ansatz ist nicht nur aus europäischer Sicht von Interesse, sondern auch in anderen Teilen der Welt relevant, wo natürliche Mischwälder durch Plantagen ersetzt wurden, die aus hochproduktiven nicht heimischen Baumarten in Reinbestandsform bestehen (Erickson et al., 2009; Kanninen, 2010). Unter Naturschutzgesichtspunkten sollte die Wiederherstellung von Mischbeständen einen großen Anteil einheimischer Arten umfassen (Pommerening und Murphy, 2004). Für viele Mischungsformen ist jedoch das Ergebnis in Bezug auf Produktivität und anderer Ökosystemgüter und -dienstleistungen ungewiss, was ihren geringen Anteil in der Praxis erklären kann (Bauhus und Schmerbeck, 2010). 


\subsection{Kronenstruktur und Mischbestände}

Die Form und Größe von Baumkronen ist ein wichtiger Aspekt in der Waldwachstumsforschung. Die Krone spielt eine zentrale Rolle für das Wachstum und die Produktivität eines Baumes. Somit ist die Erfassung der Kronenmaße ein essentieller Bestandteil für die Modellierung von Einzelbäumen und ganzen Beständen (Matthews, 1963; Mitchell, 1975). Auf Bestandesebene hängen die Waldökosysteme und deren Dynamik, Zuwachs und Ertrag maßgeblich von der Bestandesstruktur ab (Pretzsch, 2009). Die Messung der räumlichen Kronenausdehnung ist jedoch nur mit hohem zeitlichen und technischen Aufwand möglich. Deshalb wird die Erfassung der Kronen häufig auf die vertikale und horizontale Kronenausdehnung beschränkt. Aufgrund der trotzdem noch vergleichsweise umständlichen Aufnahmeverfahren bei der Vermessung von Baumkronen wird die Kronenausdehnung häufig modellbasiert hergeleitet. Je genauer die Kronenmodelle auf variable Bestandessituationen und Strukturen reagieren können, desto akkurater können die darauf folgende Vorhersagen getroffen werden (z.B. Zuwachsschätzungen) (Sprauer, 2014).

\subsubsection{Kronenbreite}

Für den allometrischen Zusammenhang von Kronenbreite mit anderen Baumparametern bei verschiedenen Baumarten gibt es Untersuchungen, welche sich auf den Brusthöhendurchmesser (BHD) (Guericke, 2001; Biber, 1996) oder aber zusätzlich noch die Baumhöhe (Condés und Sterba, 2005; Pretzsch, 2019; Schröder et al., 2005) konzentrieren. Bei dem BHD wurde bei Untersuchungen ein linearer Zusammenhang von BHD und Kronenbreite mit Bestimmtheitsmaßen von 0,7 bei Buche und Lärche (Guericke, 2001) sowie 0,9 bei Buche und Fichte (Biber, 1996) ermittelt. Bestätigung findet dieser lineare Zusammenhang in Gill et al. (2000), wobei bei mehreren Nadelbaumarten die Kronenradien modelliert und unter Einbeziehung verschiedener Prädiktoren nach Modellverbesserungen gesucht wurde. Es zeigte sich, dass der BHD der wichtigste Prädiktor des Kronenradius bei den 
meisten Baumarten ist. Dieser lineare Zusammenhang kann jedoch bei besonders kleinen Baumgrößen abweichen, sodass hier nichtlineare Funktionen zu besseren Ergebnissen führen können (Nagel et al., 2002).

\subsubsection{Mischbestände}

Die Struktur der Baumkronen beeinflusst die Verteilung von Strahlung und Niederschlag, was bei einzelnen Individuen Auswirkungen auf deren Zuwachs haben kann (Oliver et al., 1996). Als Schnittstelle zwischen terrestrischer Biomasse und der Atmosphäre spielen die Baumkronen in ihrer Form als Einzelbaumkrone, sowie in der Gesamtheit, als Kronendach eines Bestandes, eine entscheidende Rolle (Ozanne et al., 2003). Wichtige Merkmale für diese Schnittstelle in einem Waldökosystem sind Photosynthese, Respiration und Evapotranspiration. Diese funktionalen Merkmale können sich hierbei je nach Baumart unterscheiden. Eine Variation in der Ausprägung dieser Merkmale kann sich beispielsweise in einem Unterschied bei der Lichtdurchlässigkeit der Blätter, in der Kronentraufe oder einem veränderten Stammabfluss zeigen (Ameztegui et al., 2012). Die Abhängigkeit der Kronenform von der Konkurrenzsituation eines Bestandesindividuums und der Baumartenmischung sind bisher nur vereinzelt untersucht worden (Pretzsch und Schütze, 2005; Petri, 1966; Rothe, 1997; Pretzsch und Schütze, 2009; Sprauer, 2014). Die verschiedenen Konkurrenzsituationen ergeben sich durch die verschiedenen Nachbarbäume eines Baumes und deren Entfernung, Größe, Belaubung und Art. Die Art ist besonders in Mischbeständen von Bedeutung. Je nach Konkurrenzsituation können positive oder negative Effekte auf die verschiedenen Baumeigenschaften projiziert werden, was letztendlich in einem Mehr- oder Minderzuwachs resultiert. Generell wird in den hier untersuchten Mischbeständen erwartet, dass die Kombination aus Buchen und Koniferen eine höhere funktionale Diversität aufweist, als Buchenreinbestände. Zusätzlich wird angenommen, dass die damit verbunden Effekte auf die

Ökosystemfunktionen bei Beteiligung der Douglasie stärker ausgeprägt sind als in Mischung mit Fichte. Pretzsch und Schütze (2009) sowie Pretzsch et al. (2010) ha- 
ben einen Mehrzuwachs in Mischbeständen mit Buche und Fichte festgestellt. Der Mehrzuwachs ging bei Pretzsch et al. (2010) vor allem von einem besseren Wachstum der Buche aus, welche von der Beimischung der Fichte anscheinend profitieren kann. Die Mischung von Buche mit Douglasie ist bis jetzt zu dieser Fragestellung noch weniger untersucht. Empirische Erkenntnisse deuten darauf hin, dass die Rotbuche in der Regenerationsphase bei schlechten Lichtverhältnissen der Douglasie überlegen sein kann (Burschel und Huss, 2003). Die Douglasie hat in Mischung mit Buche ein besseres Wachstum und erholt sich schneller von Trockenstress, was für die Buche in der Mischung mit Douglasie hingegen nicht festzustellen ist (Thurm et al., 2016). In einer weiteren Studie wurde ein anhaltender Mehrzuwachs für Mischbestände mit Buche und Douglasie festgestellt (Lu et al., 2016). Auch die für die Produktivitätsunterschiede von Mischbeständen zugrundeliegenden strukturellen und funktionalen Merkmale wurden untersucht (Rothe und Binkley, 2001; Forrester et al., 2006). So fand man unter anderem heraus, dass sich verschiedene Baumarten mit ihren strukturellen Merkmalen (z.B. Kronenform) ergänzen können oder aber das Gegenteil der Fall sein kann (Pretzsch und Schütze, 2005). Diese Komplementarität verschiedener Baumarten in Mischbeständen wirkt sich vor allem bei der Kronenraumbesetzung eines Bestandes aus. Frech et al. (2003) fand heraus, dass die Kronenform deutlich von der Artenzugehörigkeit der Nachbarbäume beeinflusst wird. Die vertikale Aufteilung des Kronenraums durch eine asymmetrische Konkurrenz führte zu einer verbesserten Kronenraumnutzung und Koexistenz der beteiligten Baumarten.

\subsection{Ziel der Arbeit}

Ziel ist es, das Verständnis für die Struktur, das Wachstumsverhalten und die Leistung von gemischten Buchen- und Nadelbaumbeständen zu verbessern. Die Produktivität, Stabilität und Qualität von Mischständen wird maßgeblich von der Bestandesstruktur beeinflusst. Wachstumsverhalten und Wettbewerbsfähig- 
keit unter gegebenen Klima- und Standortbedingungen spiegeln sich in der horizontalen und vertikalen Struktur des Bestandes wider. Für die Untersuchung der Struktur sind Modelle äußerst nützliche Werkzeuge. Mit ihnen lässt sich ein umfassendes Verständnis für unterschiedlich zusammengesetzte Rein- und Mischbestände erhalten. Die Parametrisierung solcher Modelle erfordert einen Datensatz mit diversen Nachbarschaftsverhältnissen der enthaltenen Baumindividuen und von jedem Baum die benötigten Parameter wie z.B. Durchmesser- und Höhe und Kronenmaße. Diese Parameter sollten unter verschiedenen Konkurrenzbedingungen und räumlichen Verteilungen an den verschiedenen Standorten untersucht werden. Die so erhaltenen Informationen bilden die Grundlage für die Ableitung von Kriterien für den Anbau, Behandlung und Nutzung von Buchen - Fichten und Buchen - Douglasien Mischbeständen. Als Resultat dieser Arbeit wird angestrebt, nach Aufnahme und Untersuchung der unterschiedlichen Baumparameter ein Kronenmodell zu parametrisieren, welches auf den Versuchsflächen eine Schätzung der vertikalen Kronenform der drei Hauptbaumarten Buche, Douglasie und Fichte ermöglicht. Diese Methode soll dabei helfen, den Kronenraum genauer zu beleuchten und so auf mögliche Unterschiede von Mischbeständen im Vergleich zu Reinbeständen zu stoßen. Für die Forstpraxis ist das Wissen über diese Vor- und Nachteile von großer Bedeutung. Hier stellt sich zum Beispiel eine ökonomische Frage: „Welche Kombination von Baumarten ist am produktivsten und risikoärmsten?“. Das verbesserte Verständnis der Zusammenhänge von räumlicher Struktur und Artenzusammensetzung kann für eine Optimierung des Zuwachses in Mischbeständen verwendet werden. Zudem könnten die Ergebnisse auch dazu beitragen, für den naturschutzfachlichen Bereich relevante Fragestellungen bezüglich einer optimalen Zusammensetzung von Mischbeständen für ein arten- und strukturreiches Waldökosystem zu beantworten. Beim Vergleich von Reinbeständen mit Mischbeständen aus Fichte und Rotbuche haben Pretzsch et al. (2010) gezeigt, dass die Trockenmassenproduktion je nach Standortbedingungen zwischen $46 \%$ und $138 \%$ schwankte. Solche Mehr- und Minderzuwächse bei der Biomasseproduktion wur- 
den auch in gemischten Beständen von Eiche und Buche festgestellt (Pretzsch et al. 2013). Beide Studien basieren auf kontrollierten Feldversuchen mit Tripletts, bei denen Parzellen von Reinbeständen und Mischbeständen beider Arten nahe beieinander untersucht wurden. In dieser Arbeit durchgeführte Untersuchungen beruhen auf Versuchsflächen in Form von Quintetts. Diese lassen einen direkten Vergleich der jeweiligen Reinbestände und der Mischung Buche-Fichte und BucheDouglasie zu. Das hier vorgestellte Teilprojekt, mit dem Fokus auf die strukturelle Beschaffenheit des Kronenraumes, soll folgende Hypothesen untersuchen:

\section{Hypothesen}

- H1 Die funktionalen Merkmale von Einzelbäumen verändern sich in Abhängigkeit ihrer Mischungsform und Konkurrenzsituation. Besondere Beachtung finden Kronenmaße (Kronenbreite, Kronenlänge und Höhe der maximalen Kronenbreite) sowie die vertikale und horizontale Kronenform, deren Asymmetrie und Ovalität.

- H2 Mischungen aus Rotbuche mit Fichte oder Douglasie führen zu einer unterschiedlichen Kronenstruktur, Wuchsraumeffizienz und Produktivität im Vergleich zum Reinbestand. 


\section{Methoden und Datengrundlage}

Um die Hypothesen aus Abschnitt 1.3 untersuchen zu können, werden ertragskundliche Daten von Rein- und Mischbeständen sowie zusätzliche Strukturuntersuchungen der Baumkronen benötigt. Als Quelle hierfür dienen die im Jahr 2017 neu angelegten Versuchsflächen aus dem Graduiertenkolleg 2300 (GRK 2300), sowie Daten aus langfristigen Versuchsflächen der NW-FVA mit vergleichbaren Beständen. Hiermit wird das Zuwachsverhalten und die Entwicklung der Mischbestände analysiert.

\subsection{Ertragskundliche Aufnahmeverfahren}

Die ertragskundlichen Bestandesaufnahmen auf den Versuchsflächen des GRK2300 umfassen die Position der Stammfußpunkte, BHD, Baumhöhe, Höhe des Kronenansatzes, Höhe der maximalen Kronenbreite, sowie den Kronenradius in acht Himmelsrichtungen. Die Stammfußkoordinaten und der BHD wurden von Mitarbeitern der Universität Göttingen der Abteilung „Waldbau und Waldökologie der gemäßigten Zonen“ im Bestand aufgenommen. Die Messung der Brusthöhendurchmesser erfolgte an allen Bäumen per Umfangmaßband, die Stammfußkoordinaten wurden mit Field-Map (IFER - Monitoring and Mapping Solutions Ltd.; Czech Republic) von einer zentralen Position erfasst. Die Baumhöhe, Höhe des Kronenansatzes, astfreie Schaftlänge, sowie Höhe der maximalen Kronenbreite wurden im Rahmen dieses Teilprojektes mit dem TruPulse 360b in Kombination mit dem Criterion RD 1000 der Firma Laser Technology Inc., Colorado, gemessen. Hierzu wurde eine Position eingenommen, von welcher aus der zu vermessende Baum gut sichtbar ist und man gleichzeitig mindestens eine Baumlänge Abstand zum Baum hat. Die Vermessung mit diesen Geräten erfolgt also nicht von einer zentralen Position aus, sondern kann bei jedem Baum, je nach Sichtbarkeit, unterschiedlich sein. Die Hangneigung wurde berücksichtigt, welches bei den Geräten von Laser Technology Inc. durch die Messmethode geschieht. Hierbei werden zwei Winkel, Gerät Stammfuß, sowie Gerät - gewünschter Höhenpunkt, verwendet. Des weiteren fließt 
der Horizontalabstand zum Stamm in die Berechnung mit ein. Mit dem Horizontalabstand und dem Winkel lässt sich anschließend die Entfernung zu Stammfuß und Höhenpunkt berechnen. Aus beiden Winkeln und den zugehörigen Entfernungen kann schließlich die Höhe unter Verwendung des Kosinussatzes errechnet werden. Der Kronenansatz ist bei der Buche als die Höhe des untersten lebenden Primärastes definiert und bei der Douglasie und Fichte als die Höhe des untersten Astquirls mit mindestens drei lebenden Ästen (Kramer und Akça, 1987). Der Kronenradius wird mit Hilfe eines Kronenspiegels ermittelt (Röhle, 1986; Pretzsch et al., 2015). Es wird in acht vorgegebene Himmelsrichtungen abgelotet: Nord, Nordost, Ost, ..., Nordwest. Diese Methode wird von zwei Personen durchgeführt, eine Person stellt sich zur Ablotung an die gemessene Stelle, die andere misst die Entfernung. Die Messung der Distanz erfolgte am Stammmittelpunkt.

\subsection{Aufnahme des Kronenprofils}

Zunächst wurden selektiv Bäume anhand der erforderlichen Wuchskonstellationen (vgl. Abbildung 12) für die Aufnahme des Kronenprofils ausgewählt. Als Beispiel wurden in einem Mischbestand mit Buche und Douglasie nur Bäume ausgewählt, welche auch die jeweils andere Art als direkten Nachbarbaum aufwiesen. Die direkten Nachbarn waren das wichtigste Kriterium, da hier eine Kroneninteraktion gemessen werden sollte. Die Aufnahme des Kronenprofils wurde mit einer fotogrammetrischen Methode durchgeführt. Eine Alternative hierzu wäre eine Erfassung der Bäume mit Hilfe von Laserscans, welche für forstliche Bestandesaufnahmen schon vereinzelt Einsatz finden (Brolly et al., 2021; Danson et al., 2007). Da diese Methode allerdings einen Laserscanner und dazugehörige Software zum Auswerten der Punktwolken vorraussetzt, wurde in diesem Projekt nach einer praktikableren und zeitsparenderen Methode gesucht, welche ähnliche Informationen über die Kronenprofile gibt. Für die Erfassung des Kronenprofils wurde die Kronenfenstermethode (Albert, 1994; Hussein et al., 2000) in einer abgewandelten Form verwendet. Die ursprüngliche Methode beschreibt ein Verfahren, in dem mit Hilfe einer 
auf einem Stativ befestigten Scheibe, auf der ein Gitternetz eingebrannt ist, ein darüber gelegtes Pergamentpapier zur Aufzeichnung des Kronenumrisses genutzt wird (Gadow, 2003). Der Sinn dahinter ist, bei bekannter Länge (zum Beispiel der Baumhöhe) auf die anderen Längen (der Kronenausdehnung in verschiedenen Höhen) in der Zeichnung schließen zu können. Für diese Untersuchung wurde die Methode derart abgewandelt, dass mit einem Android-Tablet ein Foto aufgenommen wurde und die Kronenumrisse anschließend in das Foto auf dem Tablet gezeichnet wurden. Folgende Arbeitsschritte sind dafür notwendig:

1. Die Blickrichtung zum Zielbaum muss notiert werden (zum Beispiel mit einer Bussole), um später den Kronenumriss einer bestimmten Himmelsrichtung zuordnen zu können.

2. Das Foto wird von Stammfuß bis zur Spitze gemacht. Der Baum muss vollständig auf dem Bild erfasst werden, wenn die Baumhöhe als Referenzlänge für die spätere Auswertung verwendet werden soll.

3. Erfassung des Neigungswinkels von dem Tablet-Computer zum Zeitpunkt der Aufnahme. Die Entfernung zum aufzunehmenden Baum sollte möglichst weit sein, damit das Objektiv im besten Fall parallel zum Baum ausgerichtet ist. Häufig sind die Baumkronen jedoch stark verdeckt, sodass ein Kronenumriss nicht mehr erkennbar ist, oder der Baum nur teilweise aufgenommen werden kann. In diesem Fall muss die Entfernung verringert werden, bis die Krone komplett sichtbar d.h. fotografierbar wird. Die durch den hierbei sehr steilen Aufnahmewinkel entstehende Verzerrung des Bildes wird später wieder herausgerechnet.

4. Mit Hilfe einer beliebigen Zeichen-App auf dem Tablet kann nun von der selben Position aus die Krone im Foto nachgezeichnet werden. Die Position beim Einzeichnen der Kronenumrisse muss der Aufnahmeposition entsprechen, da dies die Perspektive des Objektivs war. Als Farbe zum Einzeichnen wurde hierbei der RGB-Wert $(235,0,126)$ benutzt, da dieser sich gut 
von den natürlichen Waldfarben absetzt und somit eine spätere automatische Auswertung ermöglicht wurde. Sinn hinter der sofortigen Umrandung der Krone ist, dass der Kronenumriss später am PC-Bildschirm erkennbar ist und automatisiert ausgewertet werden kann. In einem Foto mit vielen Bäumen, welche ineinander gewachsen sind, wäre es sonst unmöglich, alle Kronenbereiche einem Baum zuzuordnen. Im Bestand hingegen besteht die Möglichkeit sich zu bewegen und durch die dreidimensionale Sicht die Kronen besser auseinanderzuhalten.

Die Einträge der Blickrichtung und Neigung des Objektives müssen dem Baum zuzuordnen sein. Es sollten mindestens zwei um $90^{\circ}$ versetzte Bilder für einen Baum aufgenommen werden. So erhält man letztendlich die Kronenumrisse in 4 verschiedenen Himmelsrichtungen, da die Baumkrone auf einem Bild in jeweils zwei Himmelsrichtungen auswertbar ist.

\subsection{Korrektur der Fotos für fotogrammetrische Auswertungen}

Für die fotogrammetrische Auswertung des Bildmaterials sind mehrere Voraussetzungen zu beachten. Erstens muss eine beliebige Länge des fotografierten Objektes bekannt sein, in diesem Fall die Baumhöhe. Zweitens muss das Foto, welches nach den Gesetzen der Zentralperspektive entsteht, parallel zu dem aufzunehmenden Objekt ausgerichtet werden, damit die Linien in der Fotografie auf der selben Ebene wie die Linien des Motivs liegen (Luhmann et al., 2007). Für die Aufnahme von großen Objekten, wie Hochhäusern oder Bäumen aus geringer Entfernung, ist jedoch meistens ein Neigen des Objektives notwendig, um das gesamte Objekt zu erfassen (Abbildung 1). Durch die Neigung ist die Objektebene jedoch nicht mehr parallel zur Sensorebene, was zu Verzerrungen führt. Im oberen Teil des Bildes sind die Längen gestaucht und im unteren Teil gezerrt, sodass eine fotogrammetrische Auswertung zunächst nicht möglich ist. Eine technische Lösung für dieses Problem stellen Tilt-Shift Objektive dar, bei denen die beiden Ebenen immer parallel bleiben können. Wenn solch ein Objektiv nicht zur Verfügung steht, oder 
aber Geräte wie ein Tablet-Computer oder ein Smartphone verwendet werden, ist es auch möglich, das Bild im Nachhinein mit Bildbearbeitungssoftware zu entzerren. Hierbei wird die Stauchung und Zerrung des Bildes rückgängig gemacht, was allerdings mit einem Verlust an Bildinformationen einhergeht. Denn für eine Zerrung des Bildmaterials müssen Pixel interpoliert werden. Bei einer Stauchung gehen Pixel verloren. Dies ist jedoch nicht für die Auswertungen der Kronenumrisse von Bedeutung, da es hierbei nicht um detaillierte und optisch ansprechende Fotos geht, sondern lediglich um die eingezeichneten Kronenrandlinien. Diese lassen sich auch noch nach der Bildbearbeitung hervorragend erkennen und behalten ihre Farbinformationen in ausreichendem Umfang.

Die Korrektur der Bildneigung erfolgt mit dem Programm „ImageJ - Fiji“ anhand eines Rasters, welches über das Bild gelegt wird und es so ermöglicht, die Baumebene im Foto an diesem Raster auszurichten. Ziel ist es, die Bäume parallel zum Raster auszurichten, um keine „stürzenden Linien“ mehr im Bild vorzufinden. Die Eigenschaft von Bäumen senkrecht zu wachsen, macht die Ausrichtung anhand eines Rasters zu einer relativ schnell manuell durchzuführbaren Korrektur.

Die aufgenommenen Neigungswinkel können auch zur Korrektur des Bildes dienen. Testhalber wurde zusätzlich auch der Neigungswinkel durch das interne Gyrometer aufgezeichnet, um die Ausrichtung an einem Raster zu validieren. 

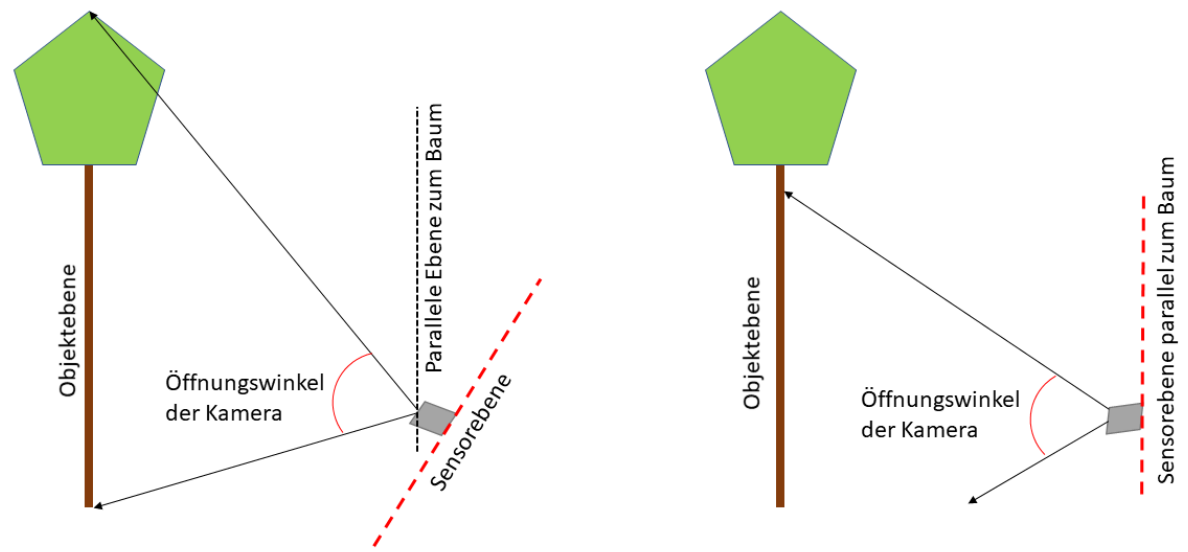

Abbildung 1: Veranschaulichung der Auswirkung einer Objektivneigung nach dem Gesetz der Zentralperspektive sowie der Problematik von Baumhöhe und Abstand für eine fotogrammetrische Aufnahme in einem Waldbestand. Links die geneigte Sensorebene, welche eine vollständige Aufnahme des Baumes ermöglicht und rechts die erwünschte parallele Sensorebene, welche jedoch dazu führt, dass nicht der gesamte Baum aufgenommen werden kann.

\subsection{Auswertung der Kronenbilder mit der Software ImageJ}

Die Kronenformen aus den korrigierten Bildern werden im folgenden Schritt digitalisiert. Hierzu wurde für Software Image ${ }^{1}$ ein Skript geschrieben, um damit die Bilder vom Baummittelpunkt bis zum eingezeichneten Kronenrand zu vermessen

\footnotetext{
${ }^{1}$ Fiji ist eine Distribution der Open-Source-Software ImageJ, die sich auf die Analyse biologischer Bilder konzentriert. Fiji kombiniert mit Hilfe moderner Softwareentwicklungsmethoden leistungsstarke Softwarebibliotheken mit einer Vielzahl von Skriptsprachen, um ein schnelles Entwickeln von Bildverarbeitungsalgorithmen zu ermöglichen. Die Software erleichtert die Umwandlung neuer Algorithmen in ImageJ-Plugins, die über ein integriertes Aktualisierungssystem für Endbenutzer freigegeben werden können. (Schindelin et al., 2012). Die Bearbeitung der aufgenommenen Fotos mit Fiji erfolgte mit einem hierfür erstelltem Skript, welches in der ImageJ Macro Language (IJM) geschrieben wurde. Wie andere Programmiersprachen verfügt IJM über grundlegende Strukturen, die zum Ausdrücken von Algorithmen verwendet werden können. Dazu gehören Variablen, Kontrollstrukturen (Bedingungs- oder Schleifenanweisungen) und benutzerdefinierte Funktionen. Darüber hinaus bietet IJM Zugriff auf alle ImageJ-Funktionen, die über die Menübefehle der grafischen Benutzeroberfläche verfügbar sind, sowie auf eine Vielzahl integrierter Funktionen, die auf die verschiedenen in ImageJ verwendeten Objekte abzielen (Bilder und Bildfenster, Regionen von Interesse, Ergebnistabelle, Diagramme, Bildüberlagerungen usw.) (Mutterer und Rasband, 2012).
} 
und dann als Punktdaten in einer Datenbank abzuspeichern. Zur Vorbereitung der Auswertung wird der umrandete Baum aus dem Bild so ausgeschnitten, dass dieser exakt in den Bildausschnitt passt. Das Bild wird dann auf eine Höhe von 1000 Pixel (die Pixelhöhe ist hierbei beliebig festgelegt, muss jedoch höher sein, als die benötigte Anzahl der Messabschnitte) unter Beibehaltung des Höhe/Breiten Verhältnis transformiert. Dieser Schritt bewirkt, dass ein Baum exakt 1000 Pixel hoch ist und somit bei bekannter Höhe die Anzahl der Pixel in eine reale Distanz umgerechnet werden kann. Wenn anschließend die Pixel vom Stamm bis zum Kronenrand gezählt werden, erhält man den Kronenradius in entsprechender Höhe als Pixelwert. Die Länge eines Pixels ist 0,1\% der Baumhöhe, da der Baum in der Höhe auf exakt 1000 Pixel eingepasst wurde. Anschließend werden mit dem Skript automatisch in 100 Höhenabschnitten des Baumes die Kronenradien gemessen. Das Skript zählt die Pixel vom Rand des Bildes bis zum ersten Auftreffen auf ein Pixel mit dem definierten RGB-Farbwert (235 $\pm 1,0,126 \pm 1$ ). Die RGB Werte wurden mit einer Schwankung von \pm 1 ausgewählt, da durch die Pixeltransformation und Entzerrung des Bildes minimale Farbveränderungen vorkommen können und der exakte Farbwert teilweise nicht mehr erkannt wurde. Als Ergebnis entstehen nun XY-Werte für das Kronenprofil eines Baumes für eine Himmelsrichtung. Die reinen Pixelwerten wurden in die realen Distanzwerte umgerechnet und in der Datenbank gespeichert. Ein Baum hat durch die Verarbeitung der Bilder anschließend vier Einträge in der Datenbank, sofern zwei Bilder aus unterschiedlichen Richtungen gemacht wurden (Abbildung 2). Für jede Himmelsrichtung sind alle ertragskundlichen Messdaten des Ausgangsbaumes sowie des Nachbarbaumes und die 100 Höhenpunkte des Kronenprofils vorhanden. 


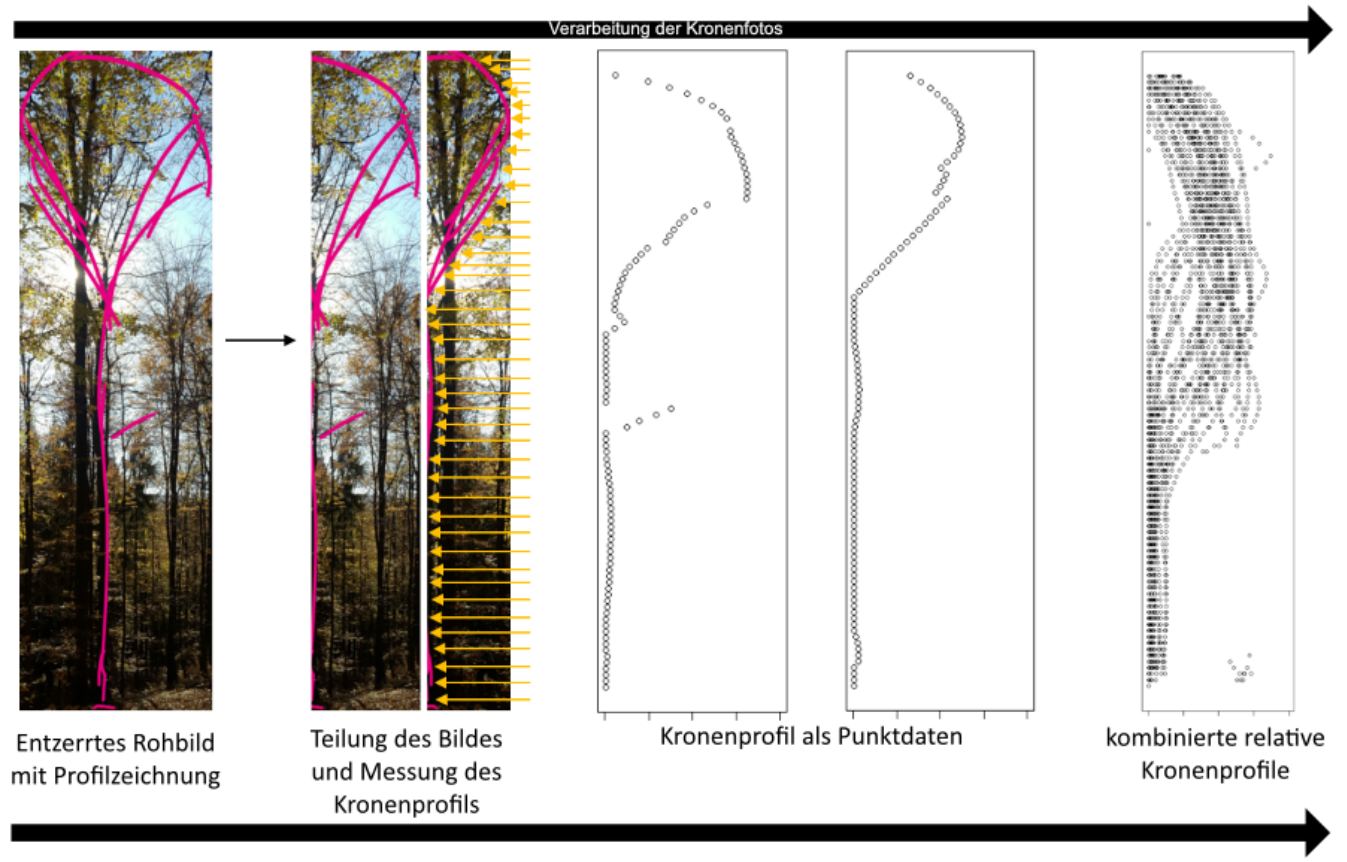

Abbildung 2: Auswertungsschritte vom Digitalfoto mit umrandeter Krone zu XY Datenpunkten, welche den Kronenrand beschreiben.

\subsection{Versuchsflächen}

\subsubsection{Farbschema und Abkürzungen der Baumarten}

In den Darstellungen wird ein einheitliches Farbschema (Tabelle 1) für die betrachteten Baumarten verwendet. Die Kurzform der Baumarten wurde auch in Mischbeständen verwendet. Ein Buchen-Douglasien Mischbestand ist Beispielsweise mit B_D abgekürzt.

Tabelle 1: Farbschema für Buche, Fichte und Douglasie

\begin{tabular}{|l|c|c|c|}
\hline Baumart & Nummer & Farbe & Kurzform \\
\hline \hline Fagus sylvatica & 211 & & B \\
\hline Picea abies & 511 & & F \\
\hline Pesudotsuga menziesii & 611 & & D \\
\hline Andere & 999 & & X \\
\hline
\end{tabular}




\subsubsection{Versuchsflächen der Nordwestdeutschen Forstlichen Versuchsan- stalt}

Die NW-FVA verfügt über viele waldwachstumskundliche Versuchsflächen, zu denen auch einige Mischbestandswuchsreihen gehören. Viele dieser Flächen wurden bereits Anfang der 1990er Jahre anlegt. Deshalb liegen hier regelmäßig erhobene Daten über einen langen Zeitraum vor. Um die Kronenuntersuchungen im Rahmen des GRK 2300 in dieser Arbeit um zusätzliche Kronendaten zu ergänzen, wurden aus dem Versuchsflächenfundus der NW-FVA die in Tabelle 2 dargestellten Einzelbäume von 156 Flächen in die Untersuchung mit einbezogen. Die Einzelbäume auf den Versuchsflächen wurden nach der Baumartenmischung und dem Vorliegen von Kronenablotungen ausgewählt, die im folgenden mit den Kürzeln B (Buche), D (Douglasie), F (Fichte) und deren Kombinationen für die Mischung angegeben werden. Beispielsweise handelt es sich bei B_D um eine Buche, in deren Einflussbereich mindestens eine Douglasie und möglicherweise weitere Buchen, aber keine anderen Baumarten stehen und bei $\mathrm{F}_{-} \mathrm{B}$ eine Fichte in Mischung mit mindestens einer Buche in ihrem Einflussbereich und ggf. weiteren Fichten. B beschreibt eine Buche mit ausschließlich Buchen in ihrem Einflussbereich. Die meisten Daten stammen von den Mischbestandswuchsreihen für Buche - Douglasie und Buche Fichte. Diese decken ein breites Spektrum an Altersklassen ab.

Tabelle 2: Übersicht über die Flächenanzahl und die darin enthaltenden Einzelbäume in deren ermittelten Mischungssituation aus der Datenbank der NW-FVA; $\mathrm{B}=$ Buche, $\mathrm{D}=$ Douglasie, $\mathrm{F}=$ Fichte

\begin{tabular}{cccccccc}
\hline Flächen & \multicolumn{10}{c}{156} \\
\hline \hline Mischung & B & B_D & B_F & D & D_B & F & F_B \\
\hline Anzahl Einzelbäume & 3963 & 1324 & 1218 & 3756 & 790 & 1190 & 841 \\
\hline
\end{tabular}




\subsubsection{Versuchsflächen GRK 2300}

Die Versuchsflächen aus dem GRK 2300 wurden gemeinsam von allen Teilprojekten in Zusammenarbeit mit den Forstämtern ausgewählt. Es wurden 8 Quintetts ausgewählt, die jeweils aus räumlich dicht aneinanderliegenden etwa gleichaltrigen Rein- und Mischbeständen der beiden

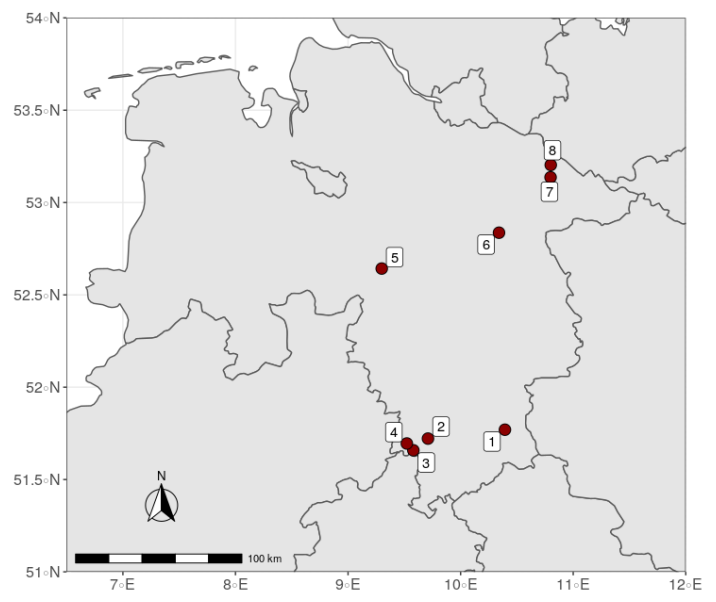

Koniferen mit Buche beste- Abbildung 3: Übersichtskarte der Versuchsflächen hen. Diese Versuchsanordnung des GRK2300 in Niedersachsen mit den Quintettsoll den Vergleich von Rein- nummern 1-8 bestand mit Mischbestand ermöglichen. Die Quintetts in Abbildung 3 befinden sich ausschließlich in Niedersachsen und sind in Tabelle 3 aufgelistet. Die Hälfte der Quintetts stockt auf Standorten im Harz und Solling. Nach Otto (1991) ist der Solling geologisch auf eine Buntsandsteinscholle zurückzuführen, welche Tertiär schildförmig aus dem Vorland aufgewölbt wurde und im Verlauf dieser Hebung zerbrach. Im Pleistozän wurde der gesamte Solling von Lößschichten überlagert, welche mit Neigung zu Pseudovergleyung sowie auf Flachhängen als Hangfließerden großflächig erhalten sind (Biber, 1996). Durch die vorherrschenden humiden Verhältnisse ist der Löß bereits vollständig entkalkt. Die Standortskraft wird aufgrund der nährstoffarmen Buntsandsteine stark durch die Lößlehmdecke bestimmt (Biber, 1996; Otto, 1991). Die höchste Erhebung des Solling liegt bei 515 m ü. NHN. Das Umland liegt bei $200 \mathrm{~m}$ ü. NHN, während das im Westen begrenzende Wesertal bei nur $100 \mathrm{~m}$ ü. NHN liegt. Das Klima im Solling ist mit vergleichsweise hohen Niederschlägen gekennzeichnet, welche aus Tabelle 3 für die GRK Flächen zu entnehmen sind. 
Im Mittel liegen die Niederschlagssummen bei 800-900 mm im Jahr, wobei ca. 400 $\mathrm{mm}$ in der forstlichen Vegetationszeit anfallen. Die verbleibenden Quintetts 5 bis 8 stehen auf Standorten in der norddeutschen Tiefebene. Diese sind wesentlich tiefer gelegen, mit einer Höhe von nur 80-160 m ü. NHN. Die Bestände befinden sich auf eiszeitlich entstandenen Landschaften im Altmoränengebiet. Auf diesen Standorten dominieren Podsole und Histosole. Diese Böden sind schwierigere Standorte, da sie einen sauren pH-Wert aufweisen, wenig Wasser pflanzenverfügbar speichern können und nur geringe Nährstoffreserven aufweisen (Blume et al., 2010). Die Niederschlagssummen liegen im Mittel bei 650-750 mm im Jahr. In Tabelle 4 werden die Mischungsanteile nach Grundfläche/Hektar beschrieben. Es ist zu erkennen, dass sich die Mischungsanteile in den jeweiligen Quintetts unterscheiden. Durch die gemeinschaftliche Auswahl der Bestände und den Anforderungen an die räumliche Gruppierung (Quintetts) war es hierbei nicht möglich, gleiche Mischungsanteile in allen Flächen zu erreichen. Somit wurden Kompromisse im Hinblick auf Gleichaltrigkeit und Grundfläche bei der Auswahl eingegangen.

Tabelle 3: Kurzcharakteristik der Quintetts des GRK 2300. Jeweilige Bestandestypen in einem Quintett: Buche, Buche-Douglasie, Douglasie, Buche-Fichte, Fichte. Standortstyp nach dem „Geländeökologischen Schätzrahmen“ (Forstplanungsamt, 2007; Forstplanungsamt und LLUR, 2009)

\begin{tabular}{cccccc}
\hline Nr. & Name & $\begin{array}{c}\text { mittl. } \\
\text { Alter }\end{array}$ & $\begin{array}{c}\text { Höhe ü. } \\
\text { NHN } \\
{[\mathrm{m}]}\end{array}$ & $\begin{array}{c}\text { Niederschl. } \\
\text { p.a. [mm] }\end{array}$ & $\begin{array}{c}\text { Standorts } \\
\text {-typ }\end{array}$ \\
\hline \hline 1 & Riefensbeek, Harz & $88 \pm 20$ & 491 & 1029 & 23.3 .2 .2 \\
\hline 2 & Dassel, Solling & $78 \pm 8$ & 426 & 821 & 9.4 .3 .5 \\
\hline 3 & Winnefeld, Solling & $73 \pm 19$ & 374 & 823 & 19.4 .2 .3 \\
\hline 4 & Nienover, Solling & $61 \pm 18$ & 343 & 880 & 23.4 .2 .3 \\
\hline 5 & Nienburg & $73 \pm 19$ & 92 & 733 & 42.3 .3 .1 \\
\hline 6 & Unterlüß & $96 \pm 18$ & 161 & 747 & 41.4 .3 .7 \\
\hline 7 & Göhrde II & $55 \pm 18$ & 130 & 682 & 43.2 .2 .2 \\
\hline 8 & Göhrde I & $66 \pm 27$ & 120 & 673 & 42.3 .3 .6 \\
\hline
\end{tabular}


Tabelle 4: Grundflächenanteile in den Mischbeständen der Quintetts als Grundfläche (Gha) pro Hektar $\left[\mathrm{m}^{2} / \mathrm{ha}\right]$ und prozentual (Anteil) [\%]

\begin{tabular}{|c|c|c|c|c|c|c|c|c|}
\hline \multirow[t]{2}{*}{ Quintett } & \multicolumn{2}{|c|}{1} & \multicolumn{2}{|c|}{2} & \multicolumn{2}{|c|}{3} & \multicolumn{2}{|c|}{4} \\
\hline & Gha & Anteil & Gha & Anteil & Gha & Anteil & Gha & Anteil \\
\hline $\mathrm{Bu}$ & 9 & $17,3 \%$ & 18,6 & $55,0 \%$ & 26,8 & $67,8 \%$ & 13,1 & $48,9 \%$ \\
\hline Dgl & 43,1 & $82,7 \%$ & 15,2 & $45,0 \%$ & 12,7 & $32,2 \%$ & 13,7 & $51,1 \%$ \\
\hline $\mathrm{Bu}$ & 14,8 & $33,4 \%$ & 17,9 & $79,6 \%$ & 19 & $71,2 \%$ & 16,4 & $36,8 \%$ \\
\hline $\mathrm{Fi}$ & 29,5 & $66,6 \%$ & 4,6 & $20,4 \%$ & 7,7 & $28,8 \%$ & 28,2 & $63,2 \%$ \\
\hline
\end{tabular}

\begin{tabular}{|c|c|c|c|c|c|c|c|c|}
\hline \multirow[t]{2}{*}{ Quintett } & \multicolumn{2}{|r|}{5} & \multicolumn{2}{|c|}{6} & \multicolumn{2}{|c|}{7} & \multicolumn{2}{|c|}{8} \\
\hline & Gha & Anteil & Gha & Anteil & Gha & Anteil & Gha & Anteil \\
\hline $\mathrm{Bu}$ & 11,9 & $30,3 \%$ & 17,1 & $52,8 \%$ & 6,6 & $26,6 \%$ & 11,6 & $29,0 \%$ \\
\hline Dgl & 27,4 & $69,7 \%$ & 15,3 & $47,2 \%$ & 18,2 & $73,4 \%$ & 28,4 & $71,0 \%$ \\
\hline $\mathrm{Bu}$ & 24,5 & $79,0 \%$ & 16,5 & $54,6 \%$ & 16,7 & $45,8 \%$ & 10,7 & $38,9 \%$ \\
\hline $\mathrm{Fi}$ & 6,5 & $21,0 \%$ & 13,7 & $45,4 \%$ & 19,8 & $54,2 \%$ & 16,8 & $61,1 \%$ \\
\hline
\end{tabular}

Im Folgenden werden die acht Quintetts einzeln vorgestellt. In den Karten sind einige Baumkronen rund und andere eckig dargestellt. Bei den eckig dargestellten Bäumen handelt es sich um Bäume, an denen im Rahmen dieser Arbeit die Kronen abgelotet wurden. Für die übrigen, rund dargestellten Bäume, wurden die Kronenbreiten aus dem BHD nach den Kronenbreitefunktionen (Döbbeler et al., 2006) abgeleitet. 


\subsubsection{Quintett 1 - Riefensbeek}

$(1.1)$
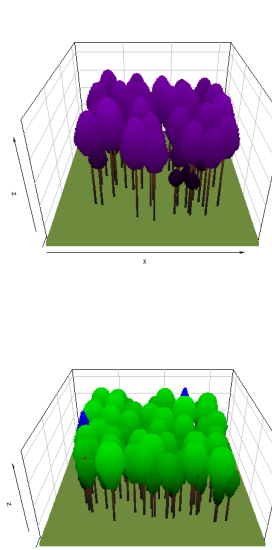

$(1.3)$

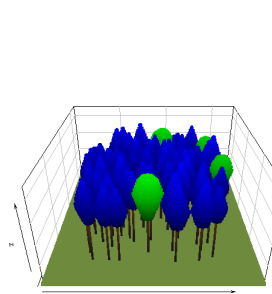

$(1.5)$
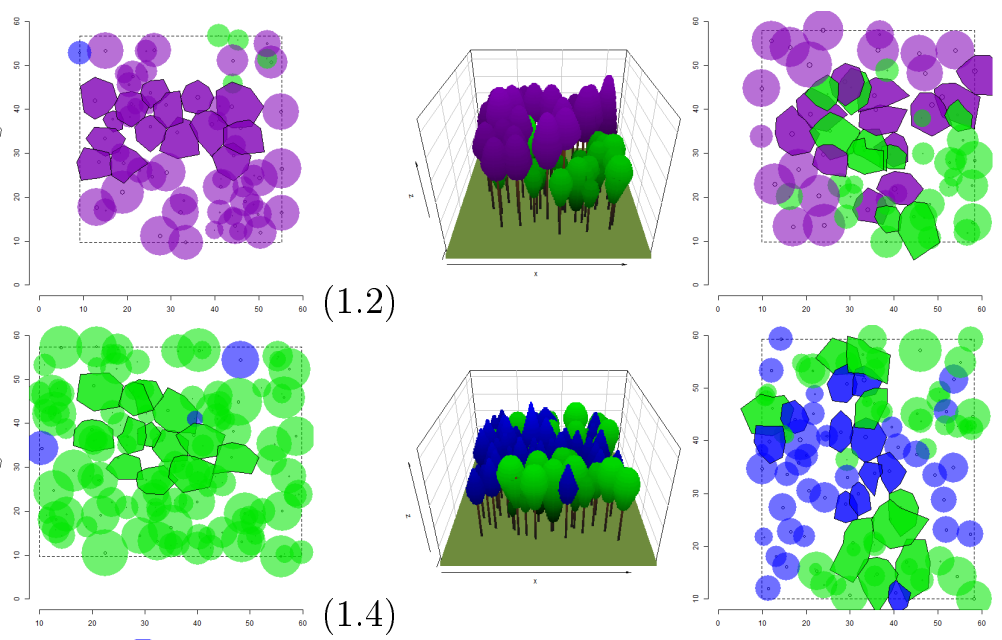

Abbildung 4: Quintett 1 mit den Flächen: (1.1) Douglasie Reinbestand 51 Jahre; (1.2) Buche-Douglasie 97/101 Jahre; (1.3) Buche-Reinbestand 101 Jahre; (1.4) Buche-Fichte 96/91 Jahre; (1.5) Fichte-Reinbestand 91 Jahre; Riefensbeek; Bestandesansicht und Kronenkarte, Fläche jeweils 0,25 ha, (Farbschema siehe Tabelle 1)

Das Quintett 1 im Forstamt Riefensbeek ist mit 491 m ü. NHN am höchsten gelegen und hat mit 1029 mm Niederschlag im Jahr auch den höchsten Niederschlag. Die Versuchsflächen diese Quintetts sind die einzigen im Harz. Der DouglasienReinbestand (1.1) ist mit 51 Jahren die jüngste Fläche von allen in diesem Quintett. Wie in der Bestandesaufrisszeichnung zu sehen ist, sind die Buchen in Fläche 1.2 größtenteils unterständig. Die Douglasien ragen mit fast der gesamten Krone über die Buchen heraus. Zudem ist die Fläche mittig einzelbaumweise durchmischt, während im Süd-Westen eine Buchengruppierung und im Nord-Osten eine Douglasiengruppierung vorhanden ist. Im Buchen-Fichten Mischbestand (1.4) ist eine gute Durchmischung vorhanden, hierbei sind vor allem mittig Fichten gruppiert 
und an den Nord- und Südseiten angrenzende Buchen mit vereinzelten Fichten.

Tabelle 5: Kennzahlen zu Quintett 1

\begin{tabular}{|c|c|c|c|c|c|c|c|c|}
\hline Fläche & Baumart & Alter & $\begin{array}{c}\text { Gha } \\
\mathrm{m}^{2} / \mathrm{ha}\end{array}$ & $\begin{array}{c}\text { Nha } \\
\mathrm{St} / \mathrm{ha}\end{array}$ & $\begin{array}{c}\mathrm{Dg} \\
\mathrm{cm}\end{array}$ & $\begin{array}{c}\mathrm{Hg} \\
\mathrm{m}\end{array}$ & $\begin{array}{c}\text { D100 } \\
\mathrm{cm}\end{array}$ & $\begin{array}{c}\text { H100 } \\
\mathrm{m}\end{array}$ \\
\hline \hline \multirow{2}{*}{1.1} & $\mathrm{D}$ & 51 & 41,2 & 236 & 47,1 & 33,2 & 64,3 & 37,8 \\
\hline \multirow{2}{*}{1.2} & $\mathrm{~B}$ & 97 & 9 & 124 & 30,4 & 26,9 & 42,5 & 30,2 \\
\cline { 2 - 10 } & $\mathrm{D}$ & 101 & 43,1 & 120 & 67,7 & 38,5 & 80,2 & 43,7 \\
\hline \multirow{2}{*}{1.3} & $\mathrm{~B}$ & 101 & 27,5 & 472 & 27,3 & 25,8 & 40,8 & 29,4 \\
\hline \multirow{2}{*}{1.4} & $\mathrm{~B}$ & 96 & 14,8 & 212 & 29,8 & 26,7 & 45,3 & 30 \\
\cline { 2 - 9 } & $\mathrm{F}$ & 91 & 29,5 & 172 & 46,7 & 29,6 & 58,9 & 32,2 \\
\hline 1.5 & $\mathrm{~F}$ & 91 & 54,6 & 348 & 44,7 & 29,2 & 55 & 31,2 \\
\hline
\end{tabular}

\subsubsection{Quintett 2 - Dassel}

$(2.1)$
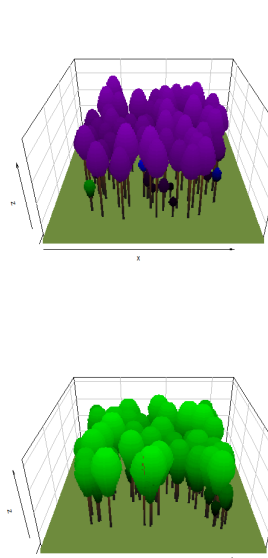

$(2.3)$

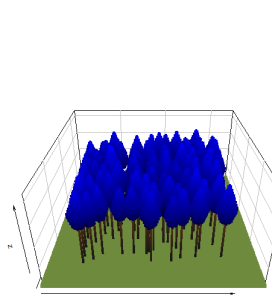

$(2.5)$

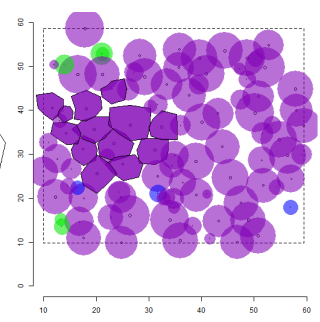

$(2.2)$
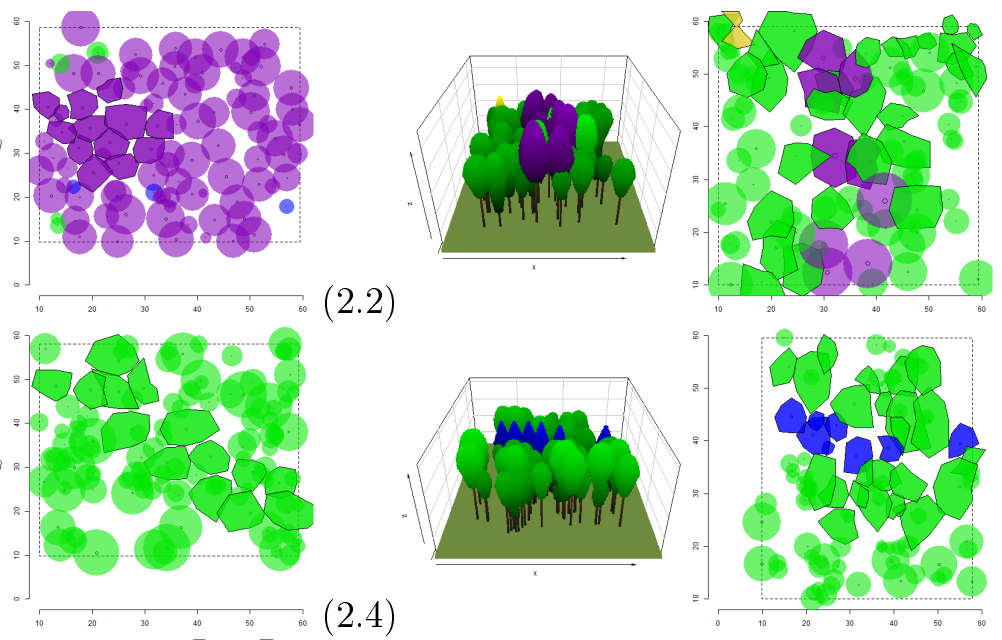

$(2.4)$
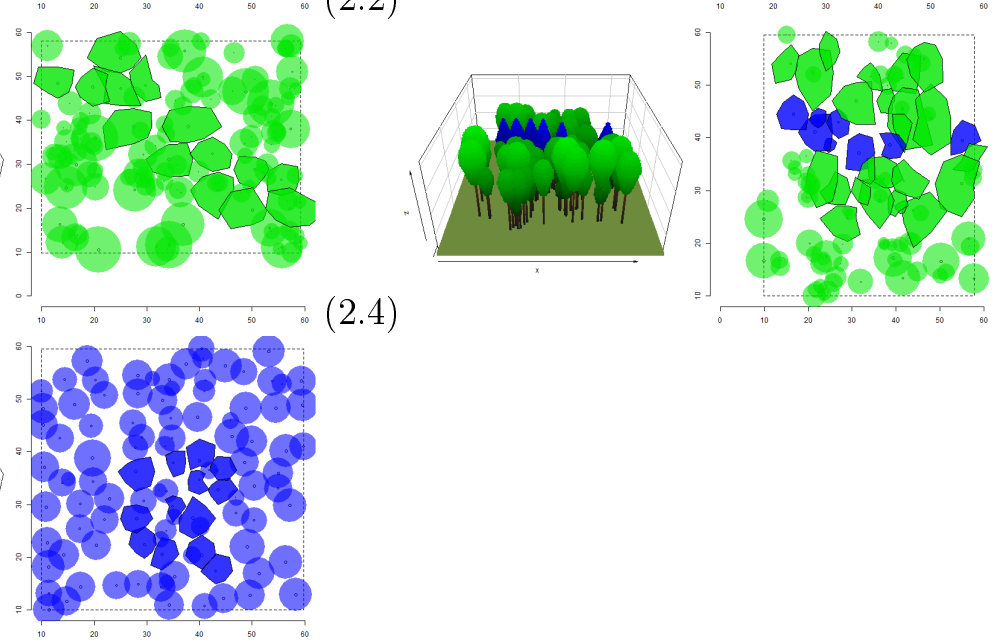

Abbildung 5: Quintett 2 mit den Flächen: (2.1) Douglasie Reinbestand 42 Jahre; (2.2) Buche-Douglasie 89/87 Jahre; (2.3) Buche-Reinbestand 88 Jahre; (2.4) Buche-Fichte 88/78 Jahre; (2.5) Fichte-Reinbestand 68 Jahre; Dassel; Bestandesansicht und Kronenkarte, Fläche jeweils 0,25 ha, (Farbschema siehe Tabelle 1) 
Das Quintett 2 zeigt bei der Douglasie in Rein- und Mischbestand einen großen Altersunterschied. Im Reinbestand (2.1) sind diese mit 42 Jahren nur halb so alt wie im Mischbestand mit 87 Jahren. Der Buchen-Douglasien Mischbestand (2.2) hat einen hohen Buchenanteil und nur ein Band von insgesamt neun großen Douglasien, welche die Mitte der Versuchsfläche durchziehen. Diese ragen zudem über die Buchen hinaus, welche mit 89 Jahren gleichaltrig sind. Ein ähnliches Bild zeigt sich im Buchen-Fichten Mischbestand (2.4). Auch hier befinden sich nur acht Fichten in der Flächenmitte, welche diese als Band durchziehen. Jedoch überragen die Fichten nicht die umstehenden Buchen. Die Fichten sind mit 78 Jahren zehn Jahre jünger als die Buchen. Die Buchen- und Fichten- Reinbestände sind in ihrem Alter vergleichbar mit den Mischbeständen und zeichnen sich durch eine homogene Verteilung der Individuen aus.

Tabelle 6: Kennzahlen zu Quintett 2

\begin{tabular}{|c|c|c|c|c|c|c|c|c|}
\hline Fläche & Baumart & Alter & $\begin{array}{c}\text { Gha } \\
\mathrm{m}^{2} / \mathrm{ha}\end{array}$ & $\begin{array}{c}\text { Nha } \\
\mathrm{St} / \mathrm{ha}\end{array}$ & $\begin{array}{c}\mathrm{Dg} \\
\mathrm{cm}\end{array}$ & $\begin{array}{c}\mathrm{Hg} \\
\mathrm{m}\end{array}$ & $\begin{array}{c}\text { D100 } \\
\mathrm{cm}\end{array}$ & $\begin{array}{c}\text { H100 } \\
\mathrm{m}\end{array}$ \\
\hline \hline 2.1 & $\mathrm{D}$ & 42 & 34,6 & 372 & 34,4 & 28,2 & 46,4 & 32,1 \\
\hline \multirow{2}{*}{2.2} & $\mathrm{~B}$ & 87 & 18,6 & 340 & 26,4 & 25,4 & 39,3 & 29,9 \\
\cline { 2 - 10 } & $\mathrm{D}$ & 89 & 15,2 & 36 & 73,3 & 39,5 & 81,5 & 39 \\
\hline 2.3 & $\mathrm{~B}$ & 88 & 24,2 & 496 & 24,9 & 24,8 & 42,4 & 28,7 \\
\hline \multirow{2}{*}{2.4} & $\mathrm{~B}$ & 88 & 17,9 & 372 & 24,8 & 24,7 & 41,3 & 26,5 \\
\cline { 2 - 10 } & $\mathrm{F}$ & 78 & 4,6 & 36 & 40,5 & 28,1 & 54,6 & 27,2 \\
\hline 2.5 & $\mathrm{~F}$ & 68 & 51,6 & 412 & 39,9 & 28 & 48,1 & 29,7 \\
\hline
\end{tabular}




\subsubsection{Quintett 3 - Winnefeld}

$(3.1)$
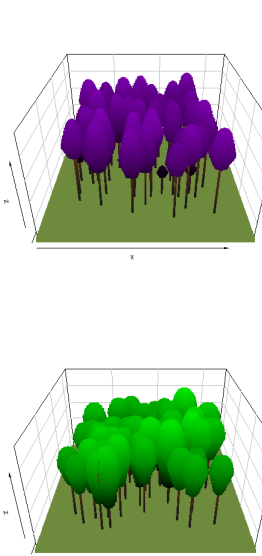

$(3.3)$

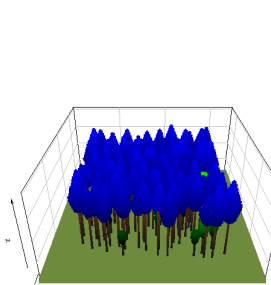

$(3.5)$
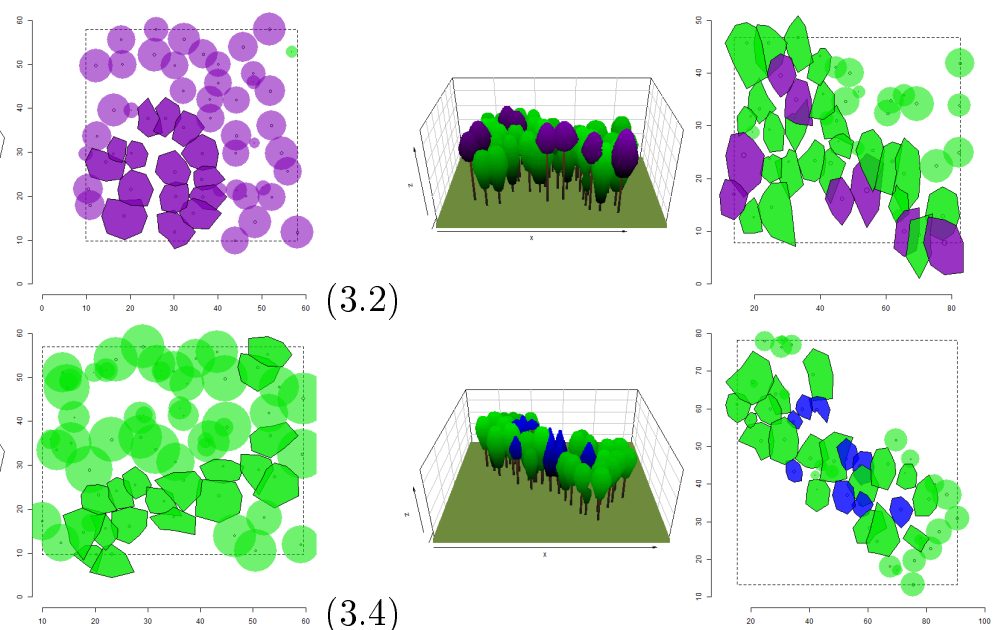

Abbildung 6: Quintett 3 mit den Flächen: (3.1) Douglasie Reinbestand 45 Jahre; (3.2) Buche-Douglasie 90/80 Jahre; (3.3) Buche-Reinbestand 90 Jahre; (3.4) Buche-Fichte 95/87 Jahre; (3.5) Fichte-Reinbestand 59 Jahre; Winnefeld; Bestandesansicht und Kronenkarte, Fläche jeweils 0,25 ha, (Farbschema siehe Tabelle 1)

Im Quintett 3 befindet sich ein 45 Jahre alter Douglasien Reinbestand (3.1), welcher im Gegensatz zu den Douglasien in der Mischung (80 Jahre) vergleichsweise jung ist. Der Mischbestand (3.2) hat hier eine längliche Ausdehnung und ist nur von acht großen Douglasien einzelbaumweise durchmischt. Ein ganz ähnliches Bild zeigt sich im Buchen-Fichten Mischbestand (3.4). Der Fichtenreinbestand (3.5) ist mit 59 Jahren jünger als die Fichten in Mischung (87 Jahre). 
Tabelle 7: Kennzahlen zu Quintett 3

\begin{tabular}{|c|c|c|c|c|c|c|c|c|}
\hline Fläche & Baumart & Alter & $\begin{array}{c}\text { Gha } \\
\mathrm{m}^{2} / \mathrm{ha}\end{array}$ & $\begin{array}{c}\text { Nha } \\
\text { St/ha }\end{array}$ & $\begin{array}{c}\mathrm{Dg} \\
\mathrm{cm}\end{array}$ & $\begin{array}{c}\mathrm{Hg} \\
\mathrm{m}\end{array}$ & $\begin{array}{c}\mathrm{D} 100 \\
\mathrm{~cm}\end{array}$ & $\begin{array}{c}\mathrm{H} 100 \\
\mathrm{~m}\end{array}$ \\
\hline \hline 3.1 & $\mathrm{D}$ & 45 & 30,2 & 216 & 42,2 & 31,5 & 52 & 34,7 \\
\hline \multirow{2}{*}{3.2} & $\mathrm{~B}$ & 90 & 21,6 & 156 & 42 & 30 & 54,5 & 33,9 \\
\cline { 2 - 9 } & $\mathrm{D}$ & 80 & 12,7 & 32 & 71 & 39,1 & 84,5 & 40,7 \\
\hline 3.3 & $\mathrm{~B}$ & 90 & 26,8 & 252 & 36,8 & 28,8 & 47,5 & 32,4 \\
\hline \multirow{2}{*}{3.4} & $\mathrm{~B}$ & 95 & 19 & 160 & 38,8 & 29,3 & 53,6 & 32,8 \\
\cline { 2 - 9 } & $\mathrm{F}$ & 87 & 7,7 & 36 & 52,2 & 30,7 & 57,5 & 33,9 \\
\hline 3.5 & $\mathrm{~F}$ & 59 & 59,3 & 532 & 34,4 & 26,3 & 42,6 & 28,5 \\
\hline
\end{tabular}

\subsubsection{Quintett 4 - Nienover}

$(4.1)$
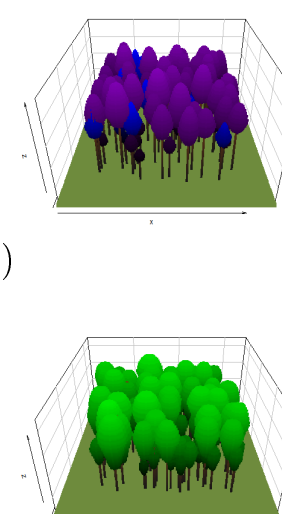

$(4.3)$
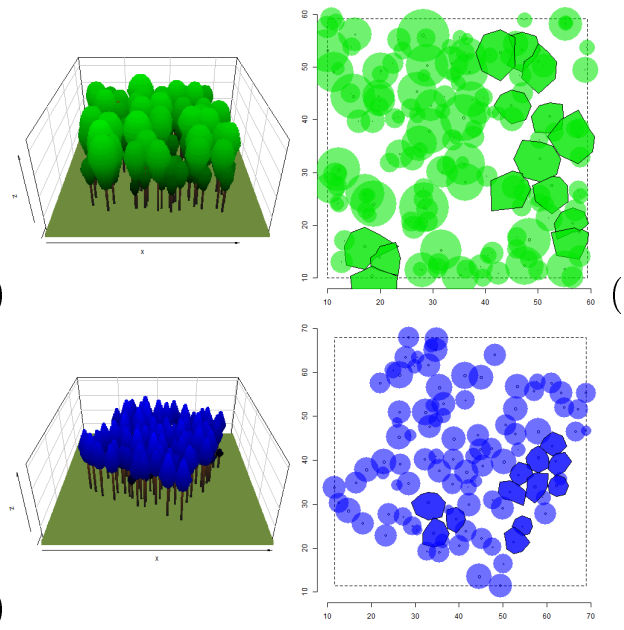
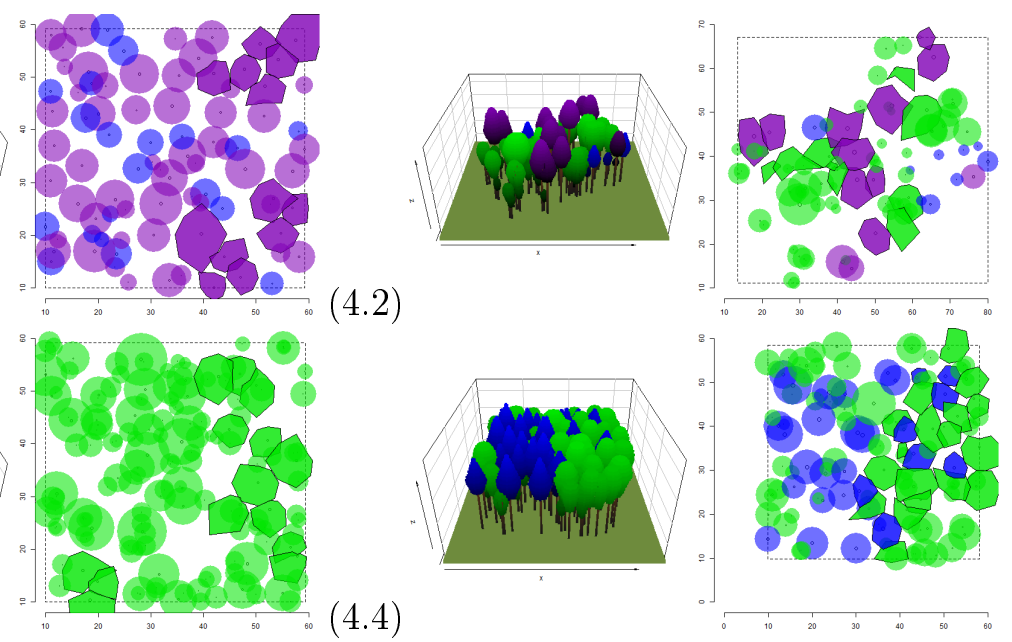

Abbildung 7: Quintett 4 mit den Flächen: (4.1) Douglasie Reinbestand 45 Jahre; (4.2) Buche-Douglasie 73/58 Jahre; (4.3) Buche-Reinbestand 87 Jahre; (4.4) Buche-Fichte 85/83 Jahre; (4.5) Fichte-Reinbestand 55 Jahre; Nienover; Bestandesansicht und Kronenkarte, Fläche jeweils 0,25 ha, (Farbschema siehe Tabelle 1)

Das Quintett 4 im Forstamt Nienover weist beim Douglasien Reinbestand (4.1) eine hohe Anzahl an unterständigen Fichten auf. Auch im Buchen-Douglasien 
Mischbestand (4.2) sind noch einzelne Fichten eingemischt. Im Unterschied zu den vorher vorgestellten Quintetts gibt es hier jedoch nur eine kleine Altersspanne der Douglasien in Rein- und Mischbestand. Die Mischbestände sind beide gut durchmischt, sodass es wenig Gruppenbildung gibt. Der Fichten Reinbestand ist mit 55 Jahren noch 28 Jahre jünger als die Fichten in Mischung.

Tabelle 8: Kennzahlen zu Quintett 4

\begin{tabular}{|c|c|c|c|c|c|c|c|c|}
\hline Fläche & Baumart & Alter & $\begin{array}{c}\text { Gha } \\
\mathrm{m}^{2} / \mathrm{ha}\end{array}$ & $\begin{array}{c}\text { Nha } \\
\text { St/ha }\end{array}$ & $\begin{array}{c}\mathrm{Dg} \\
\mathrm{cm}\end{array}$ & $\begin{array}{c}\mathrm{Hg} \\
\mathrm{m}\end{array}$ & $\begin{array}{c}\text { D100 } \\
\mathrm{cm}\end{array}$ & $\begin{array}{c}\text { H100 } \\
\mathrm{m}\end{array}$ \\
\hline \hline 4.1 & $\mathrm{D}$ & 45 & 28,1 & 260 & 37,1 & 29,5 & 50,3 & 33,7 \\
\hline \multirow{2}{*}{4.2} & $\mathrm{~B}$ & 73 & 13,1 & 256 & 25,6 & 25,1 & 42,5 & 29,9 \\
\cline { 2 - 10 } & $\mathrm{D}$ & 58 & 13,7 & 52 & 58 & 36,3 & 70,6 & 39,1 \\
\hline 4.3 & $\mathrm{~B}$ & 87 & 27,8 & 596 & 24,4 & 24,6 & 40,8 & 29,7 \\
\hline \multirow{2}{*}{4.4} & $\mathrm{~B}$ & 85 & 16,4 & 352 & 24,4 & 24,6 & 38,1 & 29,6 \\
\cline { 2 - 9 } & $\mathrm{F}$ & 83 & 28,2 & 128 & 52,9 & 30,9 & 65,6 & 32,8 \\
\hline 4.5 & $\mathrm{~F}$ & 55 & 39,6 & 404 & 35,3 & 26,6 & 44,5 & 29 \\
\hline
\end{tabular}




\subsubsection{Quintett 5 - Nienburg}

$(5.1)$
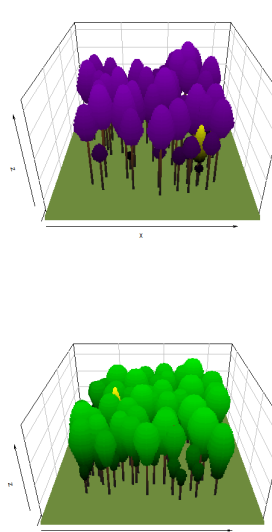

$(5.3)$

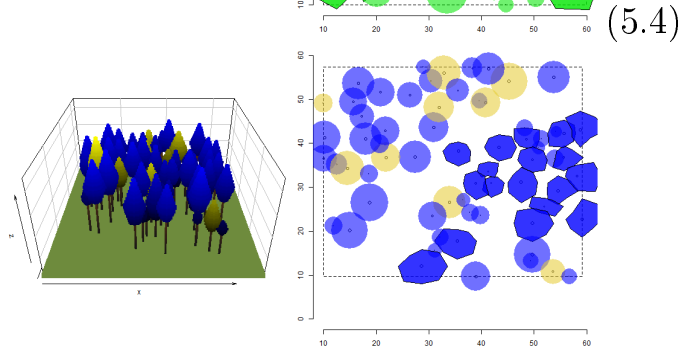

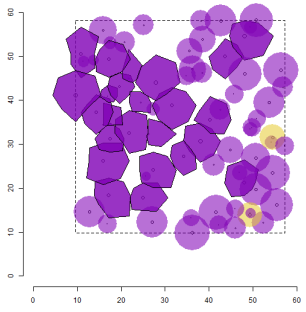
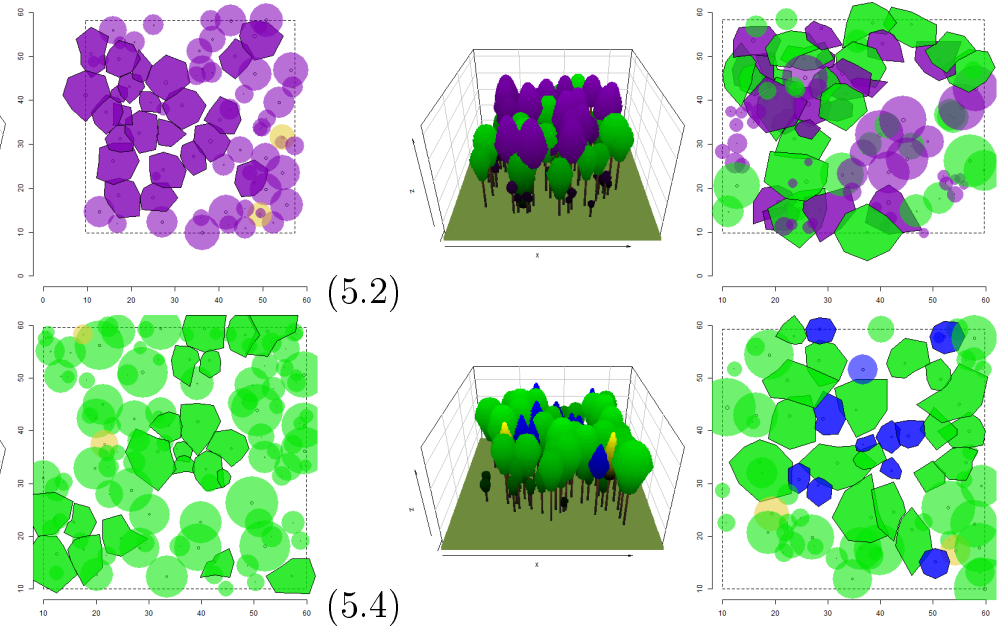

Abbildung 8: Quintett 5 mit den Flächen: (5.1) Douglasie Reinbestand 61 Jahre; (5.2) Buche-Douglasie 107/108 Jahre; (5.3) Buche-Reinbestand 78 Jahre; (5.4) Buche-Fichte 78/77 Jahre; (5.5) Fichte-Reinbestand 61 Jahre; Nienburg; Bestandesansicht und Kronenkarte, Fläche jeweils 0,25 ha, (Farbschema siehe Tabelle 1)

Der in Quintett 5 vorzufindende Mischbestand mit Buche-Douglasie (5.2) stellt einen vergleichsweise alten Bestand mit 107 und 108 jährigen Buchen und Douglasien dar. Dieser ist ganzflächig durchmischt und zeigt eine Vielzahl von Mischungssituationen. Die Douglasien ragen über die Buchen hinaus. Im BuchenFichten Mischbestand (5.4) sind nur vereinzelte und vergleichsweise kleine Fichten vorhanden. Die Kronenfläche von diesen ist im Vergleich zu den umstehenden Buchen sehr gering. Der Fichten Reinbestand (5.5) hat im südwestlichen Teil der Fläche eine Kiefernbeimischung. Hier ist vor allem der östliche Bereich der Fläche interessant, welcher eine reine Fichtengruppe beinhaltet. 
Tabelle 9: Kennzahlen zu Quintett 5

\begin{tabular}{|c|ccccccc|c|c|}
\hline Fläche & Baumart & Alter & $\begin{array}{c}\text { Gha } \\
\mathrm{m}^{2} / \mathrm{ha}\end{array}$ & $\begin{array}{c}\text { Nha } \\
\text { St/ha }\end{array}$ & $\begin{array}{c}\mathrm{Dg} \\
\mathrm{cm}\end{array}$ & $\begin{array}{c}\mathrm{Hg} \\
\mathrm{m}\end{array}$ & $\begin{array}{c}\mathrm{D} 100 \\
\mathrm{~cm}\end{array}$ & $\begin{array}{c}\mathrm{H} 100 \\
\mathrm{~m}\end{array}$ \\
\hline \hline \multirow{2}{*}{5.1} & $\mathrm{D}$ & 61 & 35,2 & 280 & 40 & 30,7 & 58,3 & 36,8 \\
\hline \multirow{2}{*}{5.2} & $\mathrm{~B}$ & 107 & 11,9 & 156 & 31,2 & 27,2 & 47 & 30,6 \\
\cline { 2 - 10 } & $\mathrm{D}$ & 108 & 27,4 & 220 & 39,8 & 30,6 & 66,7 & 37,1 \\
\hline \multirow{2}{*}{5.3} & $\mathrm{~B}$ & 78 & 27,5 & 440 & 28,2 & 26,1 & 45,4 & 30,8 \\
\hline \multirow{2}{*}{5.4} & $\mathrm{~B}$ & 78 & 24,5 & 232 & 36,6 & 28,7 & 55,3 & 33,2 \\
\cline { 2 - 9 } & $\mathrm{F}$ & 77 & 6,5 & 48 & 41,5 & 28,4 & 50,7 & 32,5 \\
\hline 5.5 & $\mathrm{~F}$ & 61 & 24,5 & 228 & 37 & 27,1 & 50,2 & 29,5 \\
\hline
\end{tabular}

\subsubsection{Quintett 6 - Unterlüß}

$(6.1)$
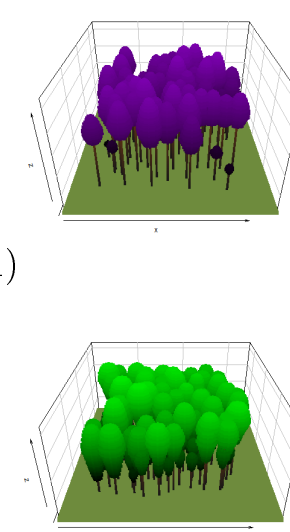

$(6.3)$

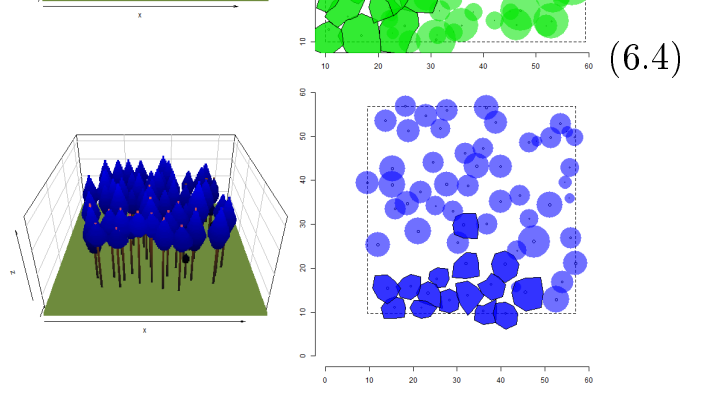

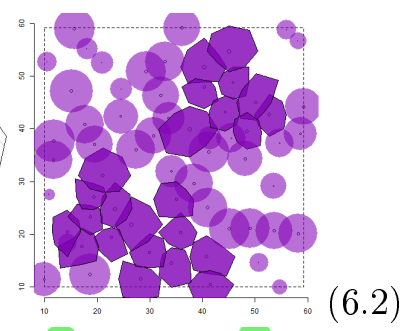
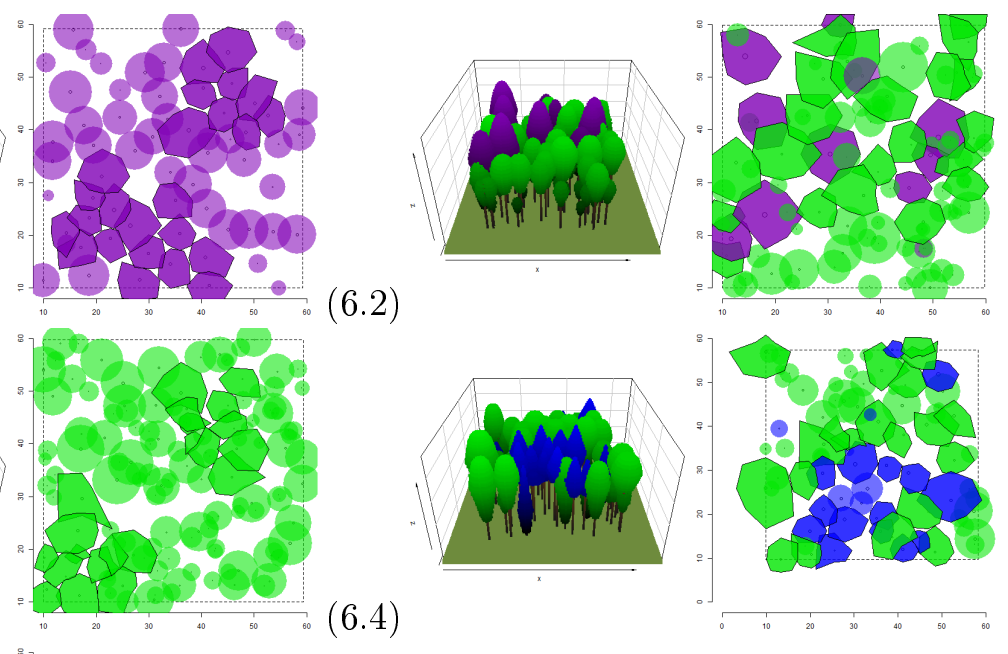

(6.2)
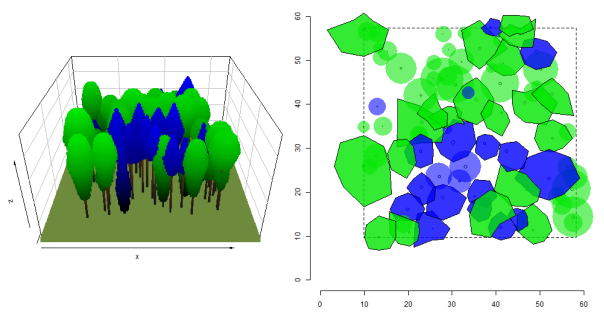

Abbildung 9: Quintett 6 mit den Flächen: (6.1) Douglasie Reinbestand 70 Jahre; (6.2) Buche-Douglasie 85/82 Jahre; (6.3) Buche-Reinbestand 85 Jahre; (6.4) Buche-Fichte 122/122 Jahre; (6.5) Fichte-Reinbestand 111 Jahre; Unterlüß; Bestandesansicht und Kronenkarte, Fläche jeweils 0,25 ha, (Farbschema siehe Tabelle 1) 
Die Flächen 6.1 bis 6.5 in Unterlüß haben mit im Mittel 96 Jahren ein vergleichsweise hohes Bestandesalter. In diesem Quintett gibt es zwischen Rein- und Mischbestand nur einen sehr kleinen Altersunterschied, was die Vergleichbarkeit positiv beeinflusst. Der Buchen-Douglasien Mischbestand (6.2) ist einzelbaumweise durchmischt mit überständigen Douglasien. Im Buchen-Fichten Mischbestand (6.4) sind im südlichen Teil die Fichten gruppiert, sodass es weniger zu einer starken Durchmischung kommt.

Tabelle 10: Kennzahlen zu Quintett 6

\begin{tabular}{|c|c|c|c|c|c|c|c|c|}
\hline Fläche & Baumart & Alter & $\begin{array}{c}\text { Gha } \\
\mathrm{m}^{2} / \mathrm{ha}\end{array}$ & $\begin{array}{c}\text { Nha } \\
\mathrm{St} / \mathrm{ha}\end{array}$ & $\begin{array}{c}\mathrm{Dg} \\
\mathrm{cm}\end{array}$ & $\begin{array}{c}\mathrm{Hg} \\
\mathrm{m}\end{array}$ & $\begin{array}{c}\mathrm{D} 100 \\
\mathrm{~cm}\end{array}$ & $\begin{array}{c}\mathrm{H} 100 \\
\mathrm{~m}\end{array}$ \\
\hline \hline 6.1 & $\mathrm{D}$ & 70 & 42,1 & 260 & 45,4 & 32,7 & 57,5 & 36,9 \\
\hline \multirow{2}{*}{6.2} & $\mathrm{~B}$ & 85 & 17,1 & 340 & 25,3 & 25 & 41,2 & 30 \\
\cline { 2 - 10 } & $\mathrm{D}$ & 82 & 15,3 & 44 & 66,6 & 38,3 & 83,6 & 38,9 \\
\hline 6.3 & $\mathrm{~B}$ & 85 & 25,4 & 528 & 24,8 & 24,7 & 39,2 & 29,2 \\
\hline \multirow{2}{*}{6.4} & $\mathrm{~B}$ & 122 & 16,5 & 228 & 30,3 & 26,9 & 47,9 & 31,1 \\
\cline { 2 - 10 } & $\mathrm{F}$ & 122 & 13,7 & 84 & 45,5 & 29,4 & 60,1 & 32,8 \\
\hline 6.5 & $\mathrm{~F}$ & 111 & 28,1 & 248 & 38 & 27,4 & 46,8 & 29,5 \\
\hline
\end{tabular}




\subsubsection{Quintett 7 - Göhrde II}

$(7.1)$
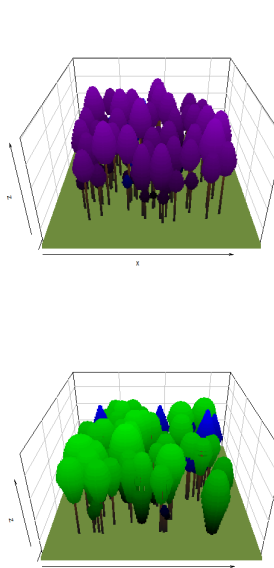

$(7.3)$
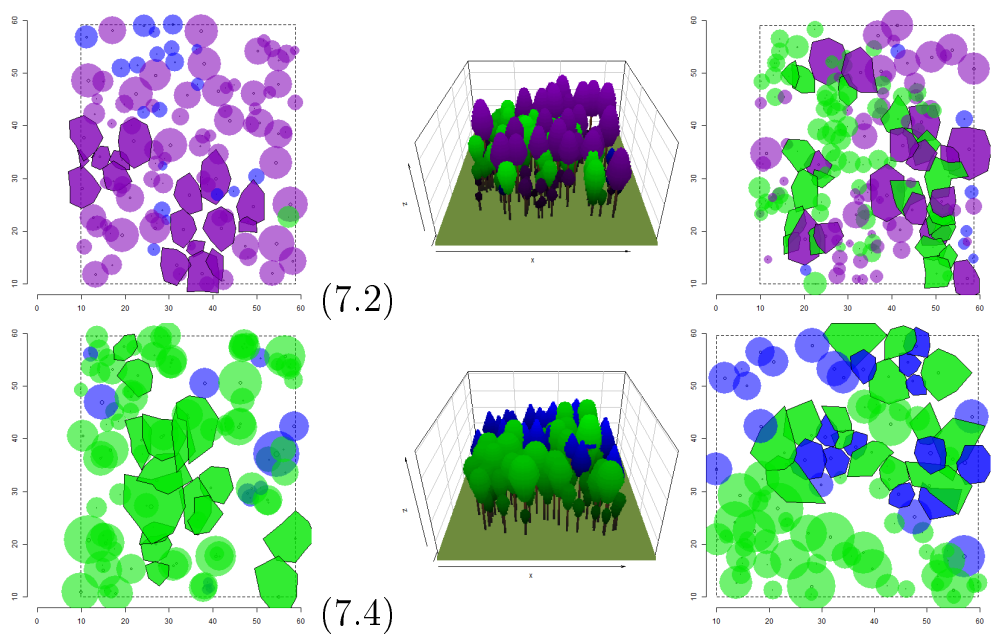

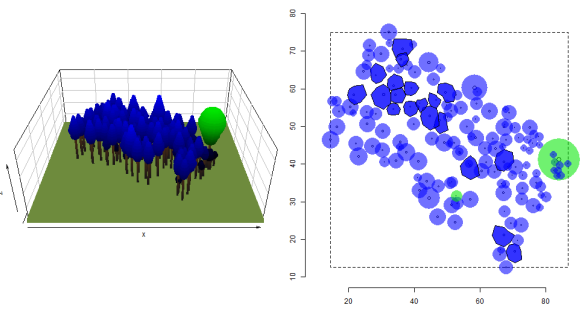

Abbildung 10: Quintett 7 mit den Flächen: (7.1) Douglasie Reinbestand 53 Jahre; (7.2) Buche-Douglasie 66/53 Jahre; (7.3) Buche-Reinbestand 96 Jahre; (7.4) Buche-Fichte 117/94 Jahre; (7.5) Fichte-Reinbestand 56 Jahre; Göhrde II; Bestandesansicht und Kronenkarte, Fläche jeweils 0,25 ha, (Farbschema siehe Tabelle 1)

In Quintett 7 sind Douglasien im Rein- sowie Mischbestand mit gleichem Alter vertreten, welches diese gut vergleichbar macht. Die Buche ist hingegen im Reinbestand älter als in der Mischung mit Douglasie. Der Buchen-Douglasien Mischbestand (7.2) ist mit einem hohen Douglasienanteil durchmischt. Diese sind stark überständig. Der Buchen Reinbestand (7.1) ist mit vereinzelten Fichten durchmischt. Im Buchen-Fichten (7.4) Mischbestand ist in südwestlichen Flächenteil eine Buchengruppierung, jedoch ist der restliche Teil der Fläche vor allem einzelbaumweise durchmischt. Die Fichten im Reinbestand (7.5) sind mit 56 Jahren wesentlich jünger als im Mischbestand (94 Jahre). 
Tabelle 11: Kennzahlen zu Quintett 7

\begin{tabular}{|c|c|c|c|c|c|c|c|c|}
\hline Fläche & Baumart & Alter & $\begin{array}{c}\text { Gha } \\
\mathrm{m}^{2} / \mathrm{ha}\end{array}$ & $\begin{array}{c}\text { Nha } \\
\mathrm{St} / \mathrm{ha}\end{array}$ & $\begin{array}{c}\mathrm{D} \\
\mathrm{cm}\end{array}$ & $\begin{array}{c}\mathrm{Hg} \\
\mathrm{m}\end{array}$ & $\begin{array}{c}\mathrm{D} 100 \\
\mathrm{~cm}\end{array}$ & $\begin{array}{c}\mathrm{H} 100 \\
\mathrm{~m}\end{array}$ \\
\hline \hline 7.1 & $\mathrm{D}$ & 53 & 29,5 & 372 & 31,8 & 26,9 & 49,6 & 33 \\
\hline \multirow{2}{*}{7.2} & $\mathrm{~B}$ & 66 & 6,6 & 328 & 16 & 19,8 & 22,5 & 23,3 \\
\cline { 2 - 10 } & $\mathrm{D}$ & 53 & 18,2 & 300 & 27,8 & 24,7 & 47,2 & 31,3 \\
\hline 7.3 & $\mathrm{~B}$ & 96 & 28,7 & 372 & 31,3 & 27,2 & 46,3 & 30,7 \\
\hline \multirow{2}{*}{7.4} & $\mathrm{~B}$ & 117 & 16,7 & 272 & 28 & 26 & 43,6 & 30,7 \\
\cline { 2 - 9 } & $\mathrm{F}$ & 94 & 19,8 & 104 & 49,2 & 30,2 & 60 & 32,6 \\
\hline 7.5 & $\mathrm{~F}$ & 56 & 33,2 & 568 & 27,3 & 23,5 & 39,2 & 27,8 \\
\hline
\end{tabular}

\subsubsection{Quintett 8 - Göhrde I}

$(8.1)$
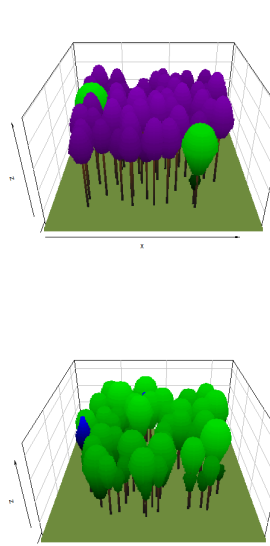

$(8.3)$

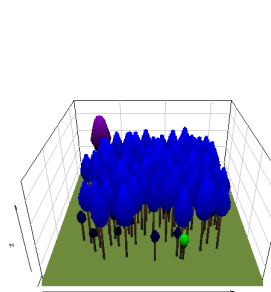

$(8.5)$

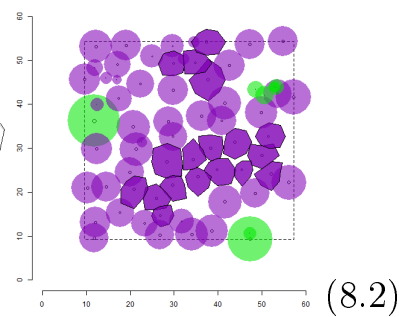

$(8.2)$

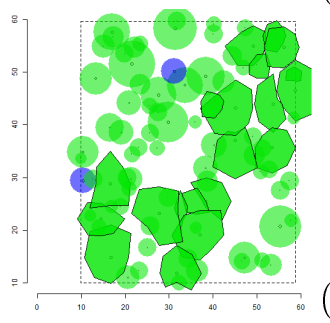

$(8.4)$
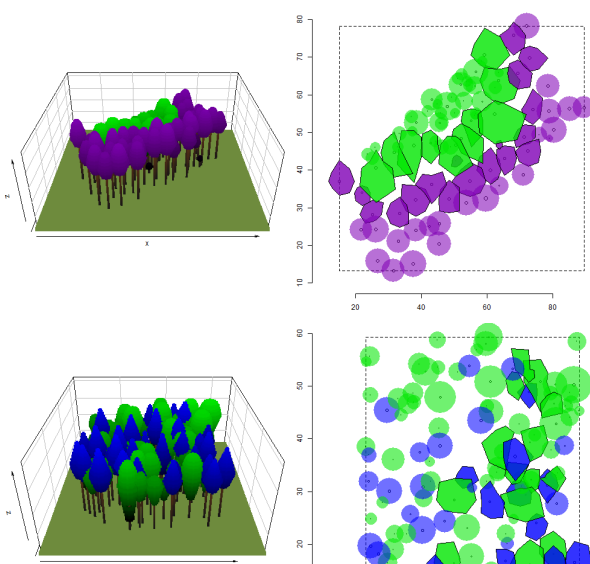
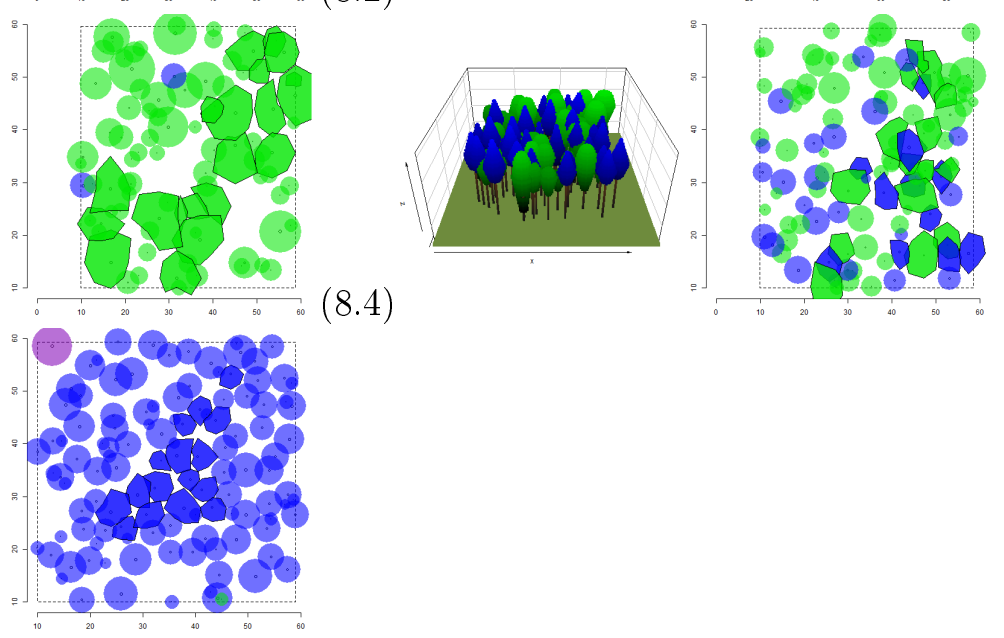

Abbildung 11: Quintett 8 mit den Flächen: (8.1) Douglasie Reinbestand 53 Jahre; (8.2) Buche-Douglasie 74/53 Jahre; (8.3) Buche-Reinbestand 130 Jahre; (8.4) Buche-Fichte 80/80 Jahre; (8.5) Fichte-Reinbestand 61 Jahre; Göhrde I; Bestandesansicht und Kronenkarte, Fläche jeweils 0,25 ha, (Farbschema siehe Tabelle 1)

Quintett 8 zeigt im Buchen-Douglasien Mischbestand (8.2) eine extreme Gruppierung der beiden Baumarten. Die einzige Interaktion zwischen den Arten findet an 
der Grenzlinie statt. Die Buchen in den beiden Mischbeständen sind jeweils um ca. 40 Jahre jünger als im Reinbestand. Der Buchen-Fichten Mischbestand zeigt eine gute, einzelbaumweise Durchmischung mit vielen Interaktionen auf. Die Fichten im Reinbestand sind nur geringfügig jünger als in Mischung (61 zu 80 Jahren).

Tabelle 12: Kennzahlen zu Quintett 8

\begin{tabular}{|c|c|c|c|c|c|c|c|c|}
\hline Fläche & Baumart & Alter & $\begin{array}{c}\text { Gha } \\
\mathrm{m}^{2} / \mathrm{ha}\end{array}$ & $\begin{array}{c}\text { Nha } \\
\mathrm{St} / \mathrm{ha}\end{array}$ & $\begin{array}{c}\mathrm{Dg} \\
\mathrm{cm}\end{array}$ & $\begin{array}{c}\mathrm{Hg} \\
\mathrm{m}\end{array}$ & $\begin{array}{c}\mathrm{D} 100 \\
\mathrm{~cm}\end{array}$ & $\begin{array}{c}\mathrm{H} 100 \\
\mathrm{~m}\end{array}$ \\
\hline \hline 8.1 & $\mathrm{D}$ & 53 & 35,2 & 248 & 42,5 & 31,6 & 51,7 & 35,2 \\
\hline \multirow{2}{*}{8.2} & $\mathrm{~B}$ & 74 & 11,6 & 176 & 28,9 & 26,4 & 47,5 & 31,7 \\
\cline { 2 - 10 } & $\mathrm{D}$ & 53 & 28,4 & 160 & 47,5 & 33,4 & 56,8 & 36,7 \\
\hline 8.3 & $\mathrm{~B}$ & 130 & 22,6 & 348 & 28,7 & 26,3 & 47,3 & 29,2 \\
\hline \multirow{2}{*}{8.4} & $\mathrm{~B}$ & 80 & 10,7 & 308 & 21 & 22,9 & 31,8 & 27 \\
\cline { 2 - 9 } & $\mathrm{F}$ & 80 & 16,8 & 140 & 39,1 & 27,7 & 48 & 29,6 \\
\hline 8.5 & $\mathrm{~F}$ & 61 & 44,4 & 460 & 35,1 & 26,5 & 45,6 & 29,5 \\
\hline
\end{tabular}

\section{6 Überlegungen zur Konkurrentenauswahl - einzelbaumbasier- te Mischungskonstellation}

Untersuchungen der Kronenstruktur fokussieren sich auf den Einzelbaum und seine ihn beeinflussenden Nachbarn. Cole und Lorimer (1994); Frech et al. (2003) haben herausgefunden, dass Einzelbäume vor allem durch die direkt angrenzenden Baumkronen beeinflusst werden und dass die Kronenform eines Baumes stark durch den Konkurrenzdruck der Nachbarbäume beeinflusst wird. Für die Umsetzung eines einzelbaumbasierten Ansatzes werden die Konkurrenten eines Baumes definiert. Diese dienen der Beschreibung von Nachbarschaftssitutation und Klassifizierung als Einzel- oder Mischbaum. Die im folgenden beschriebene Methode findet ihre Anwendung in dieser Arbeit bei der generellen Beschreibung und dem Vergleich von Baumparametern, wie z.B. der mittleren Kronenbreite in Rein- und Mischbestand. Dies trifft sowohl auf die verwendeten Daten der NW-FVA sowie die Flächen aus dem GRK2300 zu. Mit dieser einzelbaumbasierten Mischungsformerfassung war es möglich, eine große Anzahl an Flächen mit den Baumarten Buche, Fichte und Douglasie aus den Versuchsdaten der NW-FVA automatisiert 
auszuwerten. Die Alternative zum einzelbaumbasierten Ansatz wäre eine Festlegung von Einzelbäumen und ihrer Mischungsform anhand der Bestandesart. Da in vielen Mischbeständen jedoch auch Gruppen der jeweiligen Baumarten anzutreffen sind, kann es vorkommen, dass Einzelbäume nur mit der selben Baumart umgeben sind. Ihre direkte Nachbarschaft wäre dann die eines Baumes in Reinbestand.
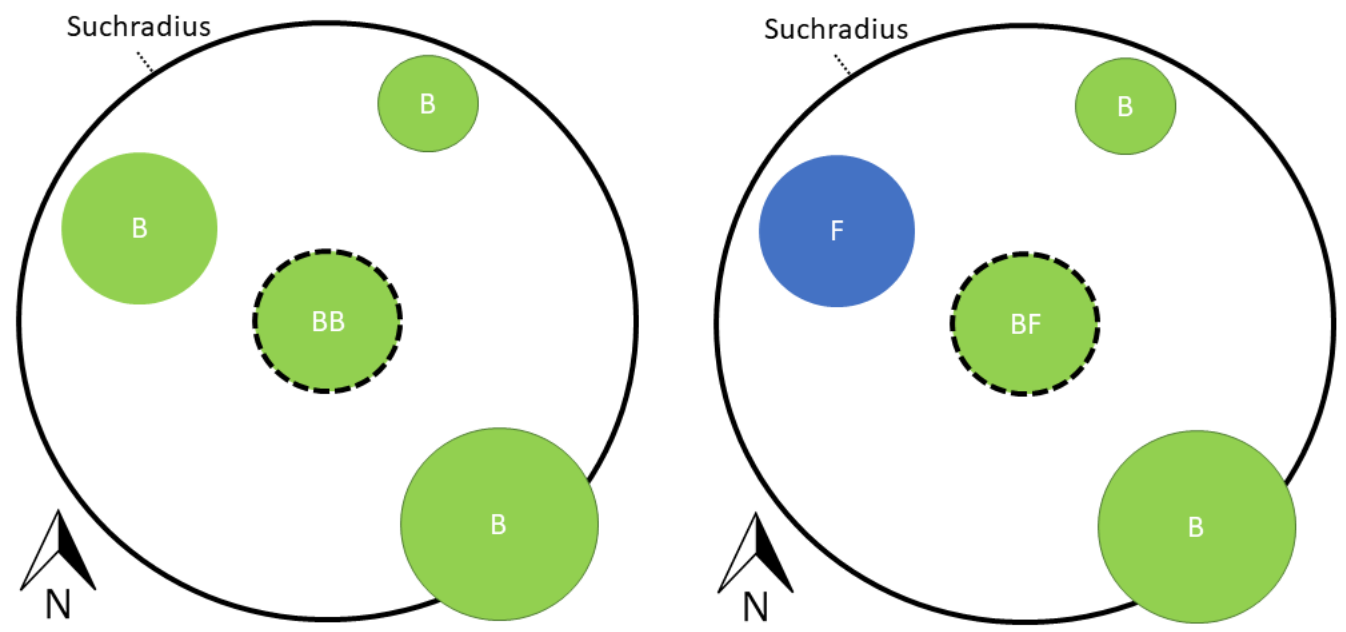

Abbildung 12: Veranschaulichung des Verfahrens zur Bestimmung der Mischungform. Links ist eine Buche umgeben von Buchen (BB) in ihrem Suchradius. Rechts ist eine Buche gemischt mit Buche und Fichte $(\mathrm{BF})$.

Um den Einfluss artfremder Konkurrenten im Sinne des Mischbestandsansatzes zu quantifizieren, werden nur solche Konstellationen als Mischung definiert, in denen Baumgruppen unterschiedlicher Arten auftreten (siehe Abbildung 12 rechts). Artreine Konstellationen (Abbildung 12 links) gelten als Reinbestände. Die Zuordnung eines Baumes zu seiner Mischungsform erfolgt durch die Erfassung der Entfernung, Größe und Art der Nachbarbäume. Die Grenzwerte für die maximale Entfernung und Mindestgröße eines Baumes, um als Konkurrent berücksichtigt zu werden, müssen zunächst festgelegt werden. In Bachmann (1996) ist eine Auflistung verschiedener Suchalgorithmen für die Auswahl von Konkurrenten bei der Ermittlung von Konkurrenzindizes zu finden. Für die Bestimmung der Mischungssituation fiel die Wahl auf die Verwendung der gemessenen mittleren Kronenradien 
beider Individuen als Kriterium für die maximale Entfernung. Es wird geprüft, ob die Entfernung der beiden Bäume größer ist, als der festgelegte Suchradius (ähnlich der Kennung KR1 in Bachmann (1996)). Die Höhe des Nachbarbaumes muss mindestens 66\% der Höhe des Ausgangsbaumes betragen, abgewandelt aus dem Konkurrenzindex CC66 (Wensel et al., 1987; Nagel, 1999). Dieses Verfahren wird dann mit dem Algorithmus um jeden Baum angewandt. Hierbei wird um den Ausgangsbaum in einem definierten Öffnungswinkel nach Konkurrenten gesucht. Der Öffnungswinkel dient dazu, in einem eingegrenzten Bereich nur jeweils den dichtesten Baum zu berücksichtigen. Weiter entfernt stehenende Bäume, welche zwar groß genug sind, werden in diesem Bereich nicht mehr berücksichtigt. Der definierte Öffnungswinkel beträgt 50 Gon. Der verbleibende Bereich um den Baum wird anschließend auf einen weiteren nächstliegenden Baum abgesucht. Dies geht so lange, bis kein Baum mehr gefunden wird, oder der gesamte Bereich abgedeckt wurde. Hierdurch entsteht eine Datenbank von Bäumen, ihren Nachbarbäumen und deren Arten, welche nach den ausgewählten Kriterien berücksichtigt wurden.

\subsection{Nachbarschaftsabhängige Kronenform}

Zur Beantwortung der Hypothese H2 soll ein Kronenmodell erstellt werden, welches in Abhängigkeit der Nachbarschaft die Kronenform eines Baumes schätzt. Die hierfür verwendeten Daten stammen aus den in 2.1 bis 2.4 beschriebenen Aufnahmeverfahren. Das gefittete Modell wird für den Aufbau eines dreidimensionalen Waldbestandes verwendet, um den Einzelbäumen eine nachbarschaftsabhängige Kronenform zu geben. Hierbei ist die Himmelsrichtung, Art und Größe des Nachbarbaumes von Bedeutung. Der Modellansatz wird in 3.2.2 beschrieben. Geschätzt werden die Parameter einer Weibull-Verteilung, welche die vertikale Kronenform abbildet und optimal durch die in Abschnitt 2.2 gemessenen Punkte verläuft. Eine ähnliche Verwendung zur Beschreibung des Kronenprofils findet die WeibullVerteilung in Mori und Hagihara (1991). Zum „fitten“ des Modells wurden die Nachbarschaftsverhältnisse der Bäume ausgewertet (Abbildung 13). Zum anschlie- 
ßenden Schätzen der Weibull-Parameter mit Hilfe des Modells sind mindestens die Stammfußkoordinate, die Baumart und der BHD erfolderlich. Aus diesen Werten können anschließend die weiteren erforderlichen Größen: Baumhöhe, Kronenansatz und Kronenradius (aus Kronenablotungen, wenn vorhanden) hergeleitet werden. Somit lässt sich das Modell auf viele Bestände übertragen, sofern diese die selben Baumartenkombinationen in vergleichbarem Alter und Größe enthalten. Bei der Herleitung der fehlenden Werte wird für den jeweiligen Baum eine Schätzfunktion (Hansen und Nagel, 2014; Döbbeler et al., 2006) verwendet um anschließend die benötigten Werte für jeden einzelnen Baum zu ergänzen. Die eigentlichen aus den Wuchskostellationen hergeleiteten Prädiktoren des Kronenmodells (Modellbeschreibung in 3.2.2 auf Seite 61) sind teilweise zusammengesetzte Größen, wie es in Abbildung 13 schematisch dargestellt ist. Beispielsweise ergibt sich die Baumdistanz aus den Stammfußkoordinaten der Bäume und der Öffnungswinkel aus dem Kronenansatz des Ausgangsbaumes und der Nachbarbaumhöhe.

Die im folgenden beschriebenen Kriterien für die Herleitung der Prädiktoren sind für das „Modellfitting“ entwickelt worden und sind eine Erweiterung des in Abschnitt 2.6 beschriebenen Verfahrens. Hierbei geht es um die Zuweisung von modellrelevaten Konkurrenten, für welche dann die Modellkrone berechnet wird (Im Vergleich hierzu geht es in Abschnitt 2.6 um die Zuordnung eines Baumes zu seiner Wuchskonstellation zum Zweck der Auswertung der Mischbestandsflächen). 


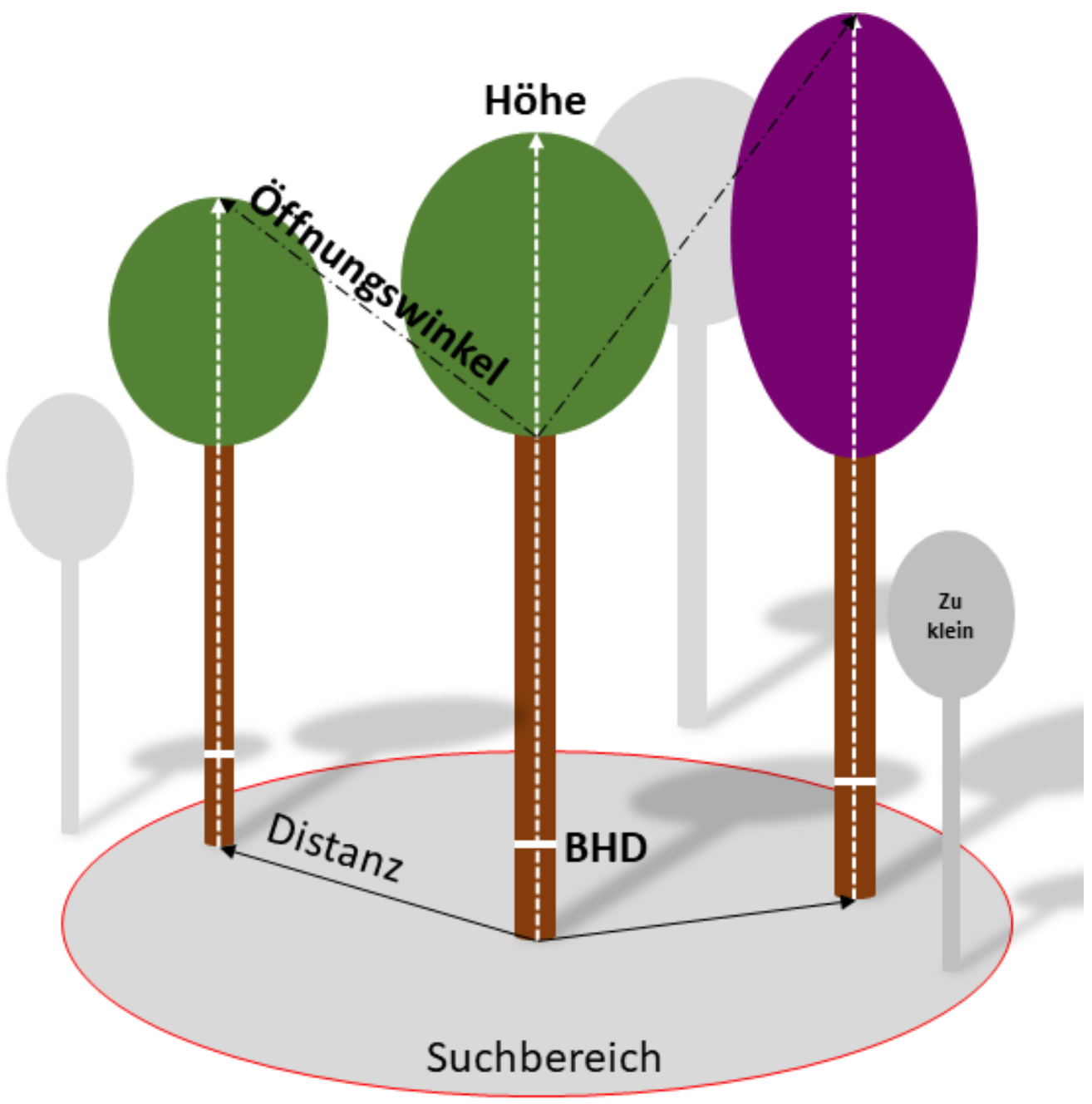

Abbildung 13: Schematische Darstellung der Erfassung der Nachbarschaftsverhältnisse eines Baumes 
Die Definition des Suchradius ist identisch mit der in Abschnitt 2.6 beschriebenen. In Abbildung 14 ist dieser dargestellt. Für die Kronenform ist die genaue Richtung des Konkurrenten wichtig. In diesem Beispiel sind das die Konkurrenten K1 - K3 mit den Richtungen (28 Gon; 152 Gon; 327 Gon), welche sich im definierten Suchradius befinden und hoch genug sind. In einem später erstellten dreidimensionalen Bestand (Abschnitt 2.8) hat die Baumkrone eine jeweils spezifische MorphoAbbildung 14: Veranschaulichung des Auswahlverfahrens von Konkurrenten logie für jede Richtung in welcher ein mit Richtungserfassung und Suchradius Konkurrent erfasst wurde. Unter der Annahme, dass die Kronenform durch den Nachbarn beeinflusst wird, sollte sie in jeder Himmelsrichtung auf den spezifischen Nachbarn reagieren. In dem hier gezeigten Beispiel hätte der Ausgangsbaum drei Konkurrenten und somit ist seine Krone aus einem Kronenmodell mit drei Parametrisierungen zusammengesetzt. Die Kronenform entspricht hierbei in Richtung eines Konkurrenten exakte dem Kronenmodell und zwischen zwei Konkurrenten einer Interpolation beider Kronenmodelle (siehe Abbildung 15). Befindet sich nur ein Konkurrent in der Nähe wird das Kronenmodell für den gesamten Baum angewandt. Dieses Vorgehen unterscheidet sich von Methoden, welche dem gesamten Baum eine symmetrische Krone aufsetzen, welche sich lediglich aufgrund der Baumgröße und Baumart im Ganzen verändern kann. Durch eine nachbarschaftsabhängige Kronenform besteht somit der Vorteil, detaillierte Strukturveränderungen darstellen zu können. 

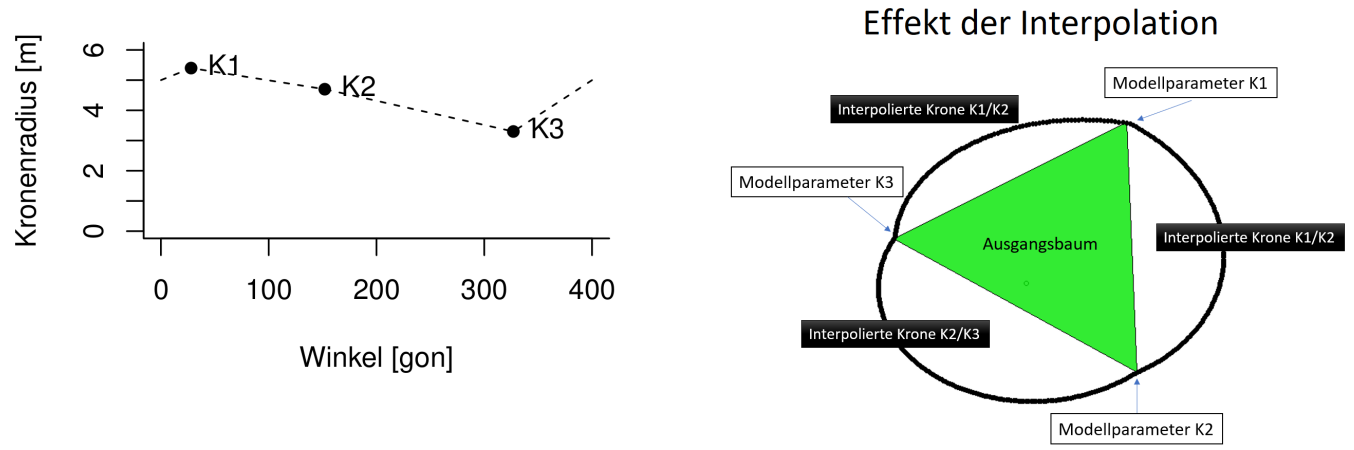

Abbildung 15: Interpolation der Krone zwischen den Punkten der zuvor bestimmten Konkurrenzbäume K1-K3 mit variierenden Modellparametern. Die lineare Interpolation (links) der Kronenradien über den Winkel erzeugt eine abgerundete Kronenform, wenn die Punkte als XY Koordinaten eingezeichnet werden.

\subsection{Aufbau eines dreidimensionalen Waldbestandes}

Zur Untersuchung der Mischungseffekte auf die Kronenform und folglich auf Bestandesparameter ist eine modellhafte Darstellung der Bäume und Bestände notwendig. Mit ihr lassen sich die situationsbedingten Eigenschaften der Kronen beschreiben. Bei Verwendung dieser Kronen auf Bestandesebende kann der Kronenraum dreidimensional vermessen werden. Ziel ist es, Kronenmantelfläche, Kronenvolumen und beleuchtete Kronenoberflächen zu bestimmen. Dafür sind die geometrisch stark variierenden Kronenformen mit ihren Überschneidungsvolumina nach Art und ihrer genauen Position im Raum zu ermitteln. Vorteil von einer solchen analytischen Möglichkeit ist, dass die räumliche Struktur der Misch- und Reinbestände gut wiedergegeben und quantifiziert werden können. Zur dreidimensionalen Darstellung und Auswertung gibt es unterschiedliche Möglichkeiten. Die erste Möglichkeit ist die Darstellung mit Hilfe von Polygonen. Mit Polygonen lassen sich beliebige Formen sehr genau darstellen, jedoch können die Berechnungen sehr aufwändig werden, wenn es bei asymmetrischen Formen um Volumenberechnungen und Zuordnung von Punkten zu den jeweiligen Objekten geht. Die zweite und geeignetere Methode für die hier durchgeführte Auswertung ist die Voxelisation des Raumes. Diese wird im Wald, bei Laserscans (Wu et al., 2013) oder auch in 
anderen Bereichen mit komplexen Körpern, wie zum Beispiel in der Computertomografie (Jones und Satherley, 2000), angewandt. Die verwendeten Kronenfunktionen sind nicht linear und die so entstehenden Waldbestände sind daher extrem komplexe Formen, welche mit der Voxelisation trotzdem gut auszuwerten sind. Die positionsgenaue Abfrage von Punkten im Raum wird mit Hilfe eines 3D-Rasters ermöglicht, in welchem man mit einer vorher bestimmten Auflösung ein Voxel mit seinen $\mathrm{x}, \mathrm{y}$ und z Koordinaten ansprechen kann. Jedem Voxel können dann beliebig viele Eigenschaften zugewiesen werden, sodass eine Abfrage für das Volumen oder die Flächen (z.B. Kronenmantelflächen) die Gesamtmenge der gesuchten Werte aufsummieren kann. Dieses kann für jede Baumart getrennt erfolgen und erlaubt im Anschluss eine Bestandesauswertung in Abhängigkeit verschiedener Mischungssituationen. Mit der Programmiersprache R lassen sich mit Hilfe des Paketes "rgl" (R Graphics Library) dreidimensionale Grafiken und Plots erzeugen. Die erzeugten Grafiken lassen sich in OpenGL ausgeben sowie unter anderem als WebGL (Web Graphics Library) exportieren, welches 3D-Grafiken hardwarebeschleunigt im Webbrowser ohne zusätzliche Erweiterungen darstellen kann.

\subsection{Voxel zur Darstellung von dreidimensionalen Objekten}

Mit der Voxeldatenstruktur wird der dreidimensionale Raum als eine Menge volumetrischer Elemente (Voxel) konzipiert und dargestellt (Abbildung 16 (1)). Ein Voxel ist ein quadratischer Baustein, dessen Größe durch die Auflösung des Voxelgitters definiert ist. Die Position eines Voxels im 3D-Voxelgittersystem wird durch

$\mathrm{x}, \mathrm{y}$ und $\mathrm{z}$ indiziert (Abbildung 16 (2)). Wird nun ein dreidimensionales Objekt mit Hilfe von Voxeln dargestellt, gilt es zunächst die Aufösung festzulegen, in welcher das Gitter erzeugt werden soll. Hierbei ist zu beachten, dass die Anzahl der Voxel exponentiell ( $\mathrm{N}=\mathrm{a}^{3} ; \mathrm{N}=$ Anzahl der Voxel, $\mathrm{a}=$ Aufösung) mit der Erhöhung der Auflösung zunimmt. Die Wahl einer zu hohen Aufösungen kann bei voxelweisen Berechnungen zu extrem hohen Laufzeiten führen. Da die Voxel eine quadratische Form haben, können andere geometrische Formen nicht exakt darge- 
stellt werden, außer die Voxel wären unendlich klein. Dies hat zur Folge, dass dem Volumen durch die Voxel immer einen gewissen Fehler anhaftet. Bei ausreichender Voxelanzahl gleichen sich die Über- und Unterschätzungen des Volumens annähernd aus $^{2}$ und mit der Vergrößerung der Auflösung, d.h. einer Verkleinerung der Voxel, wird die Abweichung noch weiter minimiert. Um ein Mittelmaß zwischen Laufzeit der Berechnung und Fehlerminimierung zu finden, wurde die Genauigkeit der einfließenden Messdaten berücksichtigt. Je nach Messmethode, Gerät, Sichtbarkeit und Bestand unterscheiden sich die Messgenauigkeiten von Höhenmessungen und schwanken zwischen 1 - $6 \%$ (Kramer und Akça, 2008; Prodan, 1965). Bei einem 30 m hohen Baum wäre somit eine Messungenauigkeit von 1,33 \% schon einer Höhenveränderung von 0,4 m. Aus diesem Grund wurde eine Voxelgröße von 0,064 $\mathrm{m}^{3}$ (Kantenlänge von $40 \mathrm{~cm}$ ) genommen, da hier die Genauigkeit ausreichend erschien, sowie die Berechnungszeit der gesamten Bestandesdarstellung noch gut durchführbar war. Die Auflösung kann stets variabel verändert werden, jedoch berufen sich die hier vorgestellten Analysen auf die Voxelauflösung von 0,064 m³.

\footnotetext{
${ }^{2}$ Dies ist abhängig von dem Auswahlverfahren der Voxelzugehörigkeit zur Krone. Wird der Voxelmittelpunkt verwendet (hier der Fall), dann gibt es Voxel außerhalb der Krone, welche mit ihren Kanten hineinragen, aber nicht gezählt werden, da ihr Mittelpunkt außerhalb der Krone liegt (und auch genau andersherum- Voxel deren Mittelpunkt innerhalb der Krone liegt, bei denen aber ein Teil nach draußen ragt). Andere Zugehörigkeitskriterien wären: Das gesamte Voxel muss innerhalb der Krone liegen - dies würde zu einer Unterschätzung des Kronenvolumens führen; Das Voxel zählt zur Krone, sobald ein Teil davon innerhalb dieser liegt - dies würde zu einer Überschätzung des Volumens führen.
} 


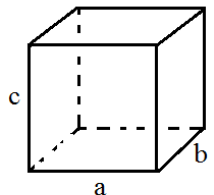

(1)

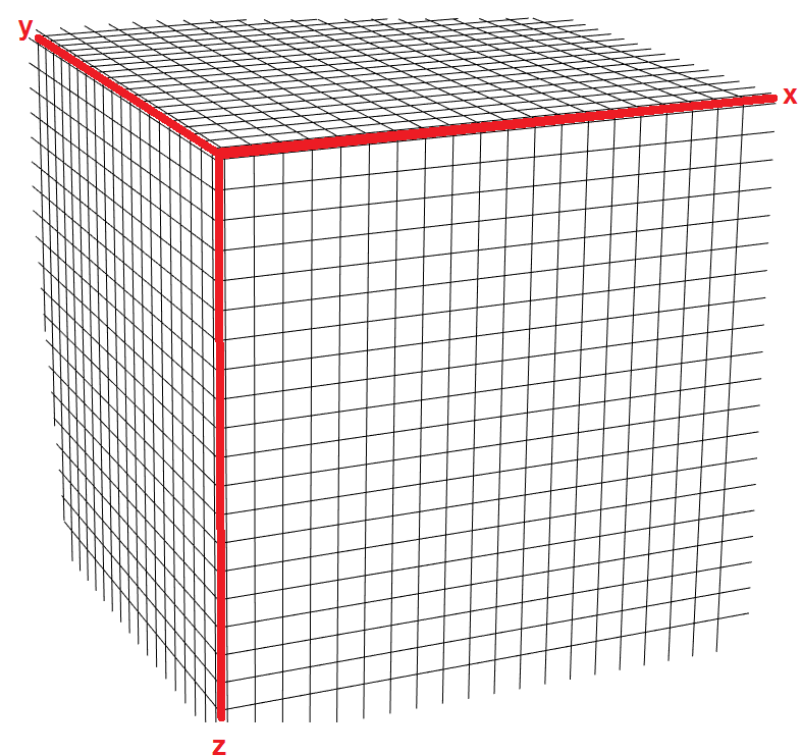

(2)

Abbildung 16: Schematische Darstellung eines einzelnen quadratischen Voxels (1) und eines Voxelgitters (2)

\subsection{Volumen- und Oberflächenberechnung mit Voxeldaten}

Bei der Volumenberechnung mit bekannter Voxelgröße reicht eine Aufsummierung der Voxel eines Körpers, um dessen Volumen relativ genau zu bestimmen. Dies gilt nur, wenn der Voxelmittelpunkt entscheidend für die Zugehörigkeit zu dem 3D-Körper ist. Ist die Anzahl der Voxel groß genug, werden sich die Voxel, welche im 3D-Körper sind und mit einem Teil herausragen, mit denen, die außerhalb sind und mit einem Teil in die Krone hineinragen, ausgleichen. Im Gegensatz dazu ist bei der Flächenberechnung von komplexen dreidimensionalen Objekten mehr zu beachten, als nur die Voxelflächen aufzusummieren. Würde man die freistehenden Randflächen der Voxel aufsummieren, hätte man eine, je nach Objektkomplexität, signifikante Überschätzung der Objektoberfläche. Die Oberfläche einer digitalisierten Voxelwelt ist grundsätzlich verschieden von der des zu Grunde liegenden Objektes (Windreich et al., 2003). Abhängig von den umgebenden Voxeln, kann sich die Fläche eines Voxels somit vergrößern. Ist der Status eines Voxels im Be- 
zug zu seinen angrenzenden Nachbarvoxel ermittelt, so kann man diesem Voxel dann einen Wert zuweisen, welcher der zugrundeliegenden geometrischen Form am ehesten entspricht. Da die Kronenfunktionen relativ glatte Oberflächen haben, wurde hierbei eine simple Anpassung zur Oberflächenberechnung verwendet. Bei komplexeren dreidimensionalen Objekten wie zum Beispiel die Verzweigungen in menschlichen Gehirnen aus 3D-Scans gibt es ähnliche Ansätze, welche jedoch noch mehr auf die verschachtelten Objekte spezialisiert sind (Windreich et al., 2003). Tabelle 13 stellt eine Übersicht über die Anzahl der Nachbarvoxel und die daraus resultierende Gewichtung für die Oberfläche des Ausgangsvoxels dar.

Tabelle 13: Voxelgewichtung in Abhängigkeit der Anzahl an Nachbarvoxeln zur Korrektur bei Oberflächenberechnungen.

\begin{tabular}{|c|c|}
\hline Anzahl Nachbarvoxel & Voxelgewichtung \\
\hline \hline 1 & 2,824 \\
\hline $2-4$ & 1,414 \\
\hline 5 & 1 \\
\hline 6 & 0 \\
\hline
\end{tabular}




\subsection{Beleuchtete Kronenmantelfäche}

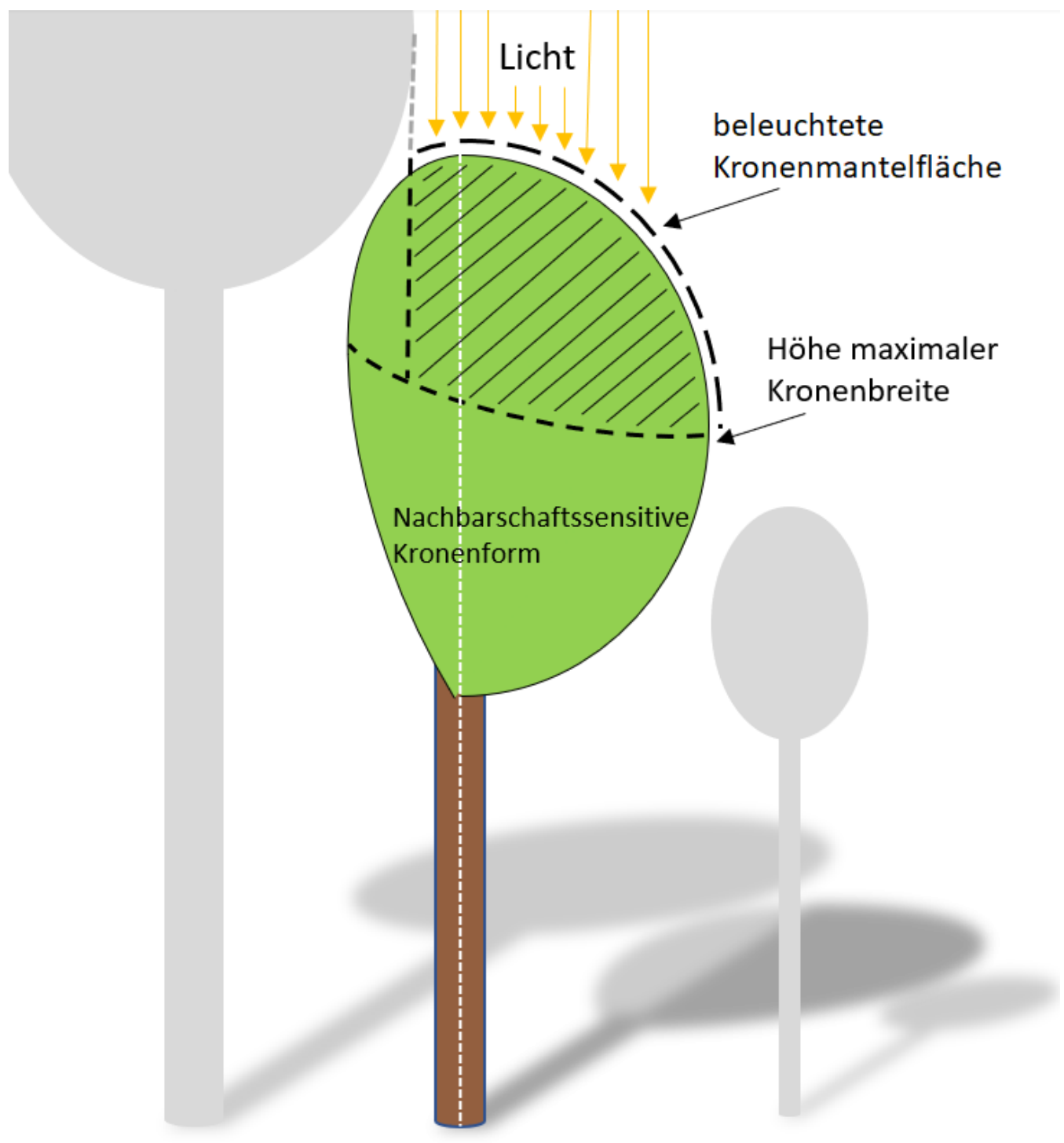

Abbildung 17: Erfassung der beleuchteten Kronenmantelfläche mit dem nachbarschaftssensitiven Kronenmodell. Die von Nachbarbäumen überdeckte Kronenmantelfläche zählt nicht zur beleuchteten Kronenmantelfläche.

Die beleuchtete Kronenmantelfläche ist definiert als Fläche, welche die Baumkrone von der Spitze bis hin zur maxmialen Kronenbreite aufweist und welche nicht durch eine Nachbarbaumkrone verdeckt ist. In Abbildung 17 wird dies mit einer asymmetrischen Baumkrone veranschaulicht. Die Auswertung des Kronenraums 
in Voxeln lässt eine richtungsbasierte Betrachtung zu. Es wird die Kronenoberfläche des asymmetrischen Kronenkörpers ausgemessen und dabei vorhandene Überschattungen der Nachbarbäume berücksichtigt. Das Ergebnis ist die beleuchtete Kronenoberfläche.

\subsection{2 Überlegungen zur Bestandesauswertung mit Hilfe des Mo- dells}

Ein Problem bei dem Vergleich der Rein- und Mischbestandsflächen ist, dass sich diese in den Quintetts trotz räumlicher Nähe zueinander im Einzelnen stark in der Grundflächenhaltung unterscheiden. Dieser Unterschied (siehe 4) führt zu einer veränderten Bestandesstruktur, die einen direkten Vergleich der realen Bestände schwer macht. Außerdem kann unterschiedliches forstliches Management zu weiteren Veränderungen der Baumattribute führen (Juchheim et al., 2017). Um dieses Problem zu lösen, wurden die Reinbestände aller Flächen als Referenz genommen und jeweils aus jedem Reinbestandspaar ein Mischbestand generiert. Hierfür kam der ForestSimulator der Norddeutschen Forstlichen Versuchsanstalt (TreeGrOSS: ForestSimulation Version 8.0.6) zum Einsatz. Als Eingabeparameter wurde die Grundfäche, Bonität nach Ertragstafel, Hg, Dg und das Alter der Reinbestände verwendet. Die Grundfläche wurde anteilsmäßig auf die gewünschte Mischung verringert (Anteilsstufen in Tabelle 14). Der erzeugte Mischbestand ist einzelbaumweise durchmischt. Die Mischbestände mit dem ForestSimulator zu generieren bietet den Vorteil, die Mischungsanteile variieren zu können, und sie mit dem Reinbestand vergleichen zu können. Tabelle 14 zeigt die für die Bestandesauswertung (Abschnitt 3.3) verwendeten Abstufungen der Mischungsanteilen nach G/ha. Das Resultat des Simulators ist eine Tabelle mit Einzelbaumwerten, welche deren Standposition und Baumparameter enthalten. Die Testbestände enthalten die relevanten Variablen wie Standpositionen und wichtige Baumkenngrößen wie

BHD, Höhe und Kronenansatz. Die Höhe und der Kronenansatz wurde hierbei aus dem BHD und der Baumart geschätzt (Hansen und Nagel, 2014; Döbbeler 
et al., 2006). Aus den Testbeständen werden dann im darauf folgenden Schritt mit einem R-Skript die 3D-Voxelbestände generiert. Von diesen Voxelbeständen werden anschließend das Kronenvolumen, die Kronenoberfläche, Kronenschirmfläche oder die beleuchtete Kronenoberfläche berechnet. Hierzu wurden vier verschiedene Funktionen erstellt, welche die jeweilige Berechnung in den Voxelräumen durchführen.

Tabelle 14: Abstufungen der Mischungen für den Vergleich der generierten Mischbestände mit den realen Reinbeständen.

\begin{tabular}{|c|c|c|}
\hline Grundflächenverhältnis & $\mathrm{Bu}$ & Dgl oder Fi \\
\hline \hline $1: 3$ & $25 \%$ & $75 \%$ \\
\hline $1: 1$ & $50 \%$ & $50 \%$ \\
\hline $3: 1$ & $75 \%$ & $25 \%$ \\
\hline
\end{tabular}

\subsection{Verwendete Software $\mathbf{R}$}

$\mathrm{R}$ ist eine Sprache und Umgebung für statistische Berechnungen und Grafiken. Es ist ein GNU-Projekt, das der S-Sprache und -Umgebung ähnelt, die von John Chambers und Kollegen in den Bell Laboratories (ehemals AT \& T, jetzt Lucent Technologies) entwickelt wurde. R bietet eine Vielzahl statistischer (lineare und nichtlineare Modellierung, klassische statistische Tests, Zeitreihenanalyse, Klassifizierung, Clustering usw.) und grafischer Techniken und ist in hohem Maße erweiterbar. R kam in fast jedem Arbeitsschritt zum Einsatz, da hier einerseits die aufgenommenen Daten verwaltet und sortiert und zum anderen die folgenden Berechnungen mit dieser Sprache umgesetzt wurden. Mit R lassen sich die in der Nordwestdeutschen Forstlichen Versuchsanstalt vorhandenen Datenbanken auslesen, welche im .mdb Format (Microsoft Access Database) vorliegen. Dies geschieht mithilfe des R-Paketes „RODBC“ (Ripley, 2012), welches die Möglichkeiten von ODBC (Open DataBase Connectivity) in der R-Umgebung verfügbar macht. Für die statistische Modellierung wurde das Paket „mgcv“ Wood (2001) 
verwendet. „mgcv“ bietet Funktionen für die verallgemeinerte additive Modellierung und die verallgemeinerte additive gemischte Modellierung. Der Begriff GAM schließst jedes Modell ein, das von unbekannten Glättungsfunktionen der Prädiktoren abhängt und durch quadratisch penalisierte Wahrscheinlichkeitsmaximierung geschätzt wird.

Eine Vielzahl von den verwendeten Funktionen wurden in einem R-Paket zusammengefasst. Die einzelnen Auswertungsschritte, welche in dieser Arbeit vorgenommen wurden, sind mit diesem Paket implementiert. Dass Flussdiagramm (Abbildung 18) zeigt die einzelnen Module, welche zur Erstellung und Auswertung der Bestände benötigt werden und in welcher Reihenfolge diese nacheinander ausgeführt wurden. 


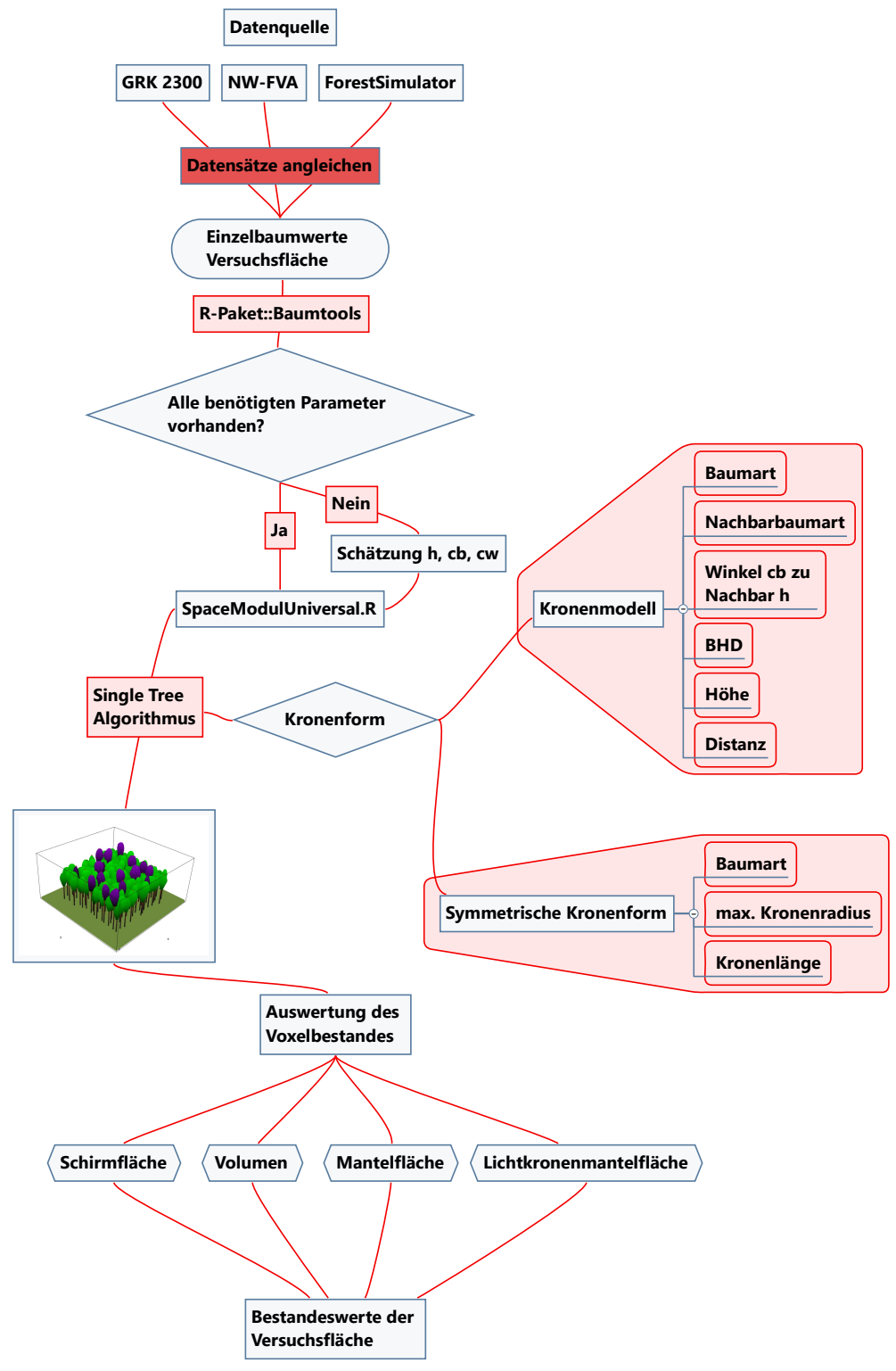

Abbildung 18: Flussdiagramm der verschiedenen Auswertungsschritte mit den erstellten Funktionen. 


\section{Ergebnisse}

\subsection{Kronenmaße und Allometrien in Rein- und Mischbestand}

\subsubsection{Zusammenhang von BHD und Kronenbreite}

In Abbildung 19 ist der modellierte Zusammenhang von BHD und mittlerer Kronenbreite (arithmetisches Mittel aus den acht abgeloteten Kronenradien) dargestellt. Daten für diese Untersuchung stammen aus dem Datensatz der NW-FVA und den GRK2300 Versuchsflächen. Die Zuordnung der Bäume zu Rein- und Mischbestand erfolgte nach dem in Abschnitt 2.6 beschriebenen Verfahren. Die Grafik zeigt den Unterschied zwischen den verschiedenen Baumarten sowie zwischen Rein- und Mischbeständen der jeweiligen Baumarten. Die Douglasien (violette Farben) sowie die Fichten (blaue Farben) zeigen in Rein- und Mischbestand keinen signifikanten Unterschied in ihrer Kronenbreite. Die Regressionsfunktionen mit den eingezeichneten Konfidenzintervallen zeigen bei den Koniferen nur geringe Abweichungen voneinander und keinen offensichtlichen Trend. Hingegen ist bei den Buchen in den beiden Mischungsformen (grüne Farben) bis zu einem BHD von $63 \mathrm{~cm}$ eine signifikant größere Kronenbreite vorhanden, als bei den Buchen im Reinbestand (Dunkelgrün). Dies bedeutet, dass die Buchen bei gleichem BHD eine im Mischbestand breitere Krone aufweisen. Zwischen den Baumarten ist, wie zu erwarten, ein großer Unterschied zu erkennen. Buchen haben die größte Kronenbreite und Douglasien haben eine geringfügig größere Kronenbreite als Fichten bei jeweils gleichem BHD. Die im oberen Teil horizontal ausgerichteten Boxplots veranschaulichen die Datenlage der verschiedenen Baumarten und Mischungen. Hier unterscheiden sich vor allem die Daten der Fichten, da der Hauptteil vergleichsweise große BHDs haben $(30-50 \mathrm{~cm})$, während bei Buche und Douglasie auch viele Daten mit kleinerem BHD existieren (15-50 cm). Insgesamt ist die Datenlage der Buchen und Douglasien in Reinbestandssituation am höchsten, welche in beiden Fällen fast 4000 Individuen umfasst. 


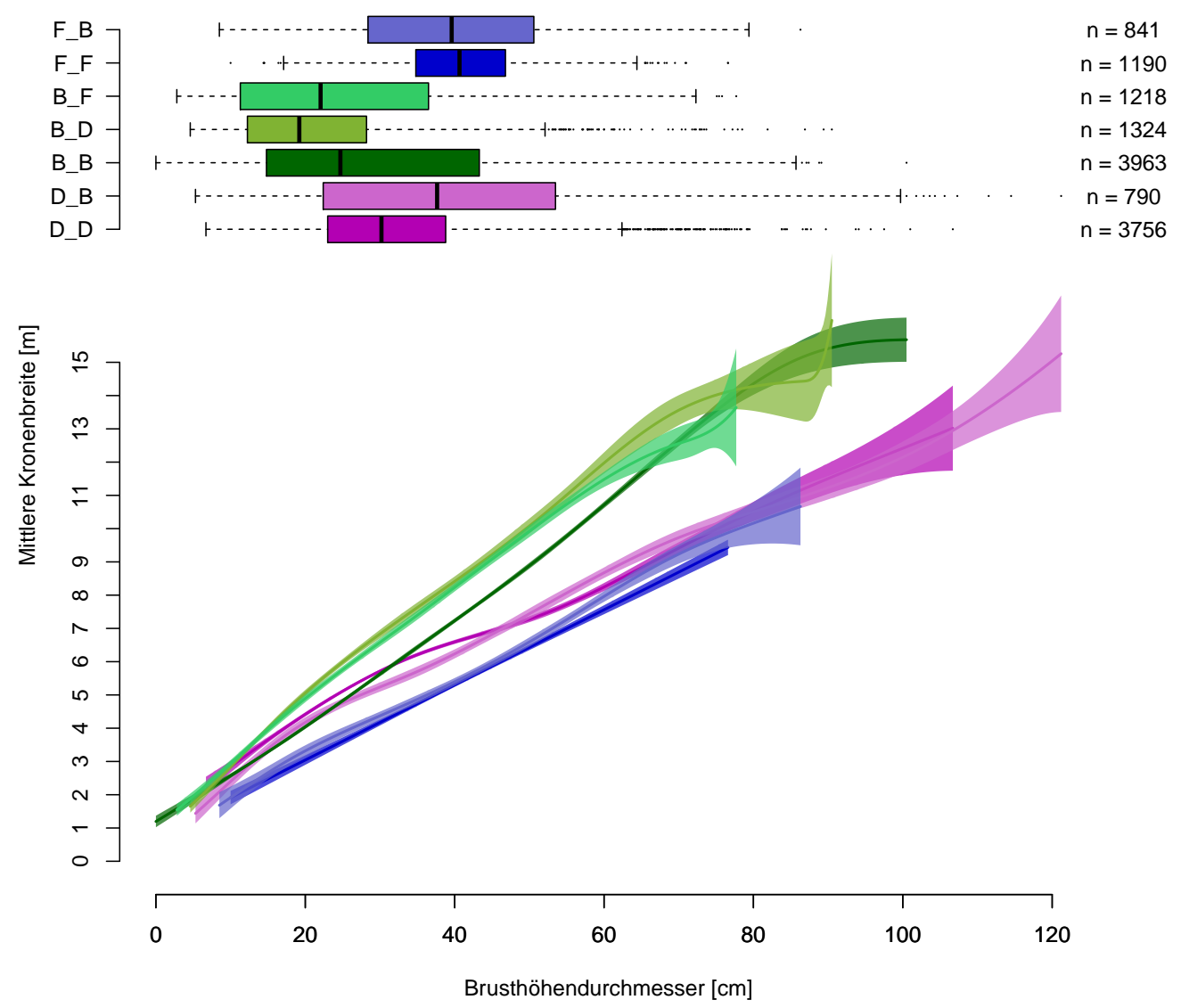

Abbildung 19: Modellierter Zusammenhang von BHD und mittlerer Kronenbreite, aufgeteilt in die verschiedenen Mischungsformen. Die Parameter des additiven Regressionsmodells (mit Annahme normalverteilter Residuen) mit monoton steigenden Effekten wurden mit Hilfe der Funktion ,scam“ aus dem R-Paket SCAM (Pya und Wood, 2015) geschätzt.

\subsubsection{Höhe der maximalen Kronenbreite}

Die Untersuchung der Höhe der maximalen Kronenbreite beruht auf den Daten der NW-FVA und GRK2300, welche auch in Abschnitt 3.1.1 beschrieben wurden. Das Ergebnis ist in Abbildung 20 als Wahrscheinlichkeitsdichtefunktion dargestellt. Diese wird als Kerndichtefunktion (Parzen-Fenster-Methode) aus den diskreten Daten der Messungen abgeleitet. Es beschreibt damit die Häufigkeit der gemessenen Werte der Höhe der maximalen Kronenbreite. Auf der x-Achse ist in 
den Abbildungen a-c die nach Grundfläche angepasstene relative Höhe verwendet worden. In den Plots d-e sind die absoluten Höhen verwendet worden, um einen Rückschluss auf die Bestandesstruktur zu ermöglichen. In Mischung mit Douglasie hat die Buche die niedrigste relative Höhe der maxialen Kronenbreite. Die Douglasien- (b) und Fichtenkronen (c) zeigen nur minimale Unterschiede in Reinund Mischbestand. Die Wahrscheinlichkeitsdichtefunktionen von jeweils Rein- und Mischbaum haben ihren Höchstwert beide in ähnlicher Höhe von 0,78 der relativen Baumhöhe. In (d) sind die Höhen der maximalen Kronenbreiten von Buchen und Douglasien nebeneinander aufgezeichnet. Hierbei ist zu erkennen, dass der Höchstwert der Buchenkronen unter der Douglasie liegt. Die Buchenkrone hat die breiteste Stelle ihrer Krone (bei $23 \mathrm{~m}$ ) mit höchster Wahrscheinlichkeit unter der der Douglasie $(28 \mathrm{~m})$. Ein anderes Bild zeigt sich beim Vergleich der Buchenkronen mit den Fichtenkronen (e). Hier ist die maximale Kronenbreite beider Baumarten häufig auf selber Höhe (23 m). 
Buchenkrone (a)

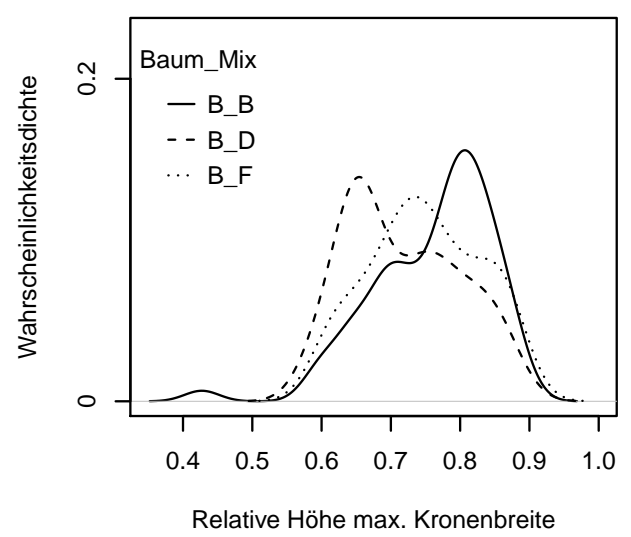

Fichtenkrone (c)

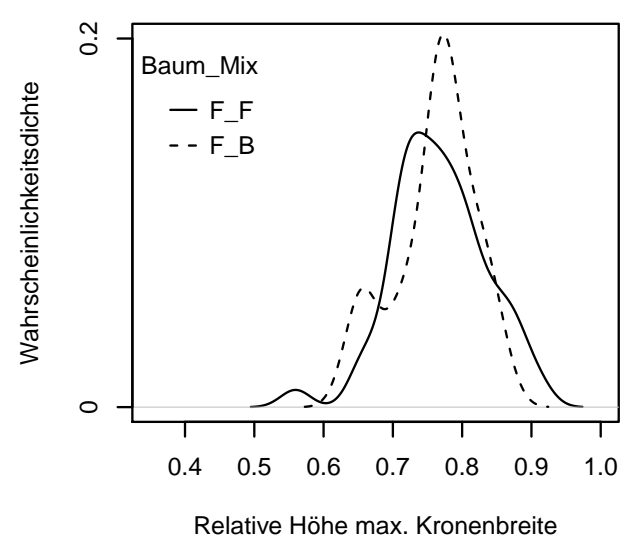

Vergleich Buche-Douglasie (d)

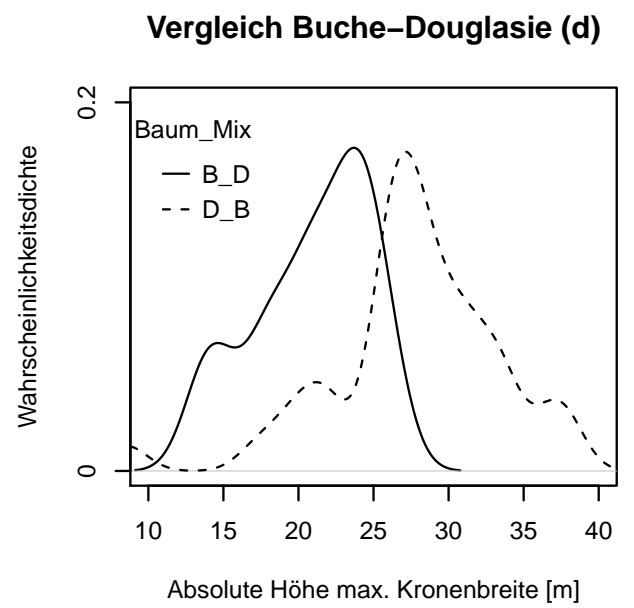

\section{Douglasienkrone (b)}

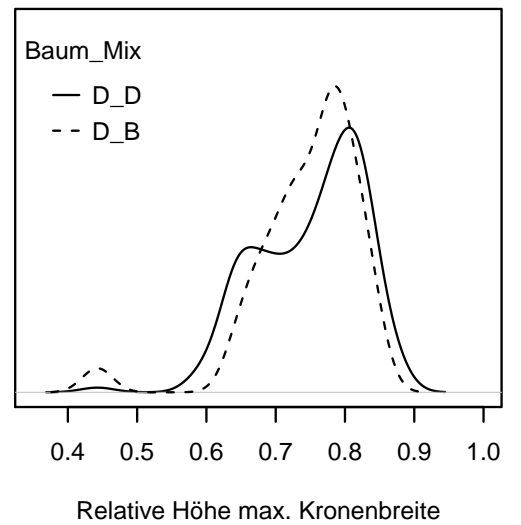

Relative Höhe max. Kronenbreite

\section{Vergleich Buche-Fichte (e)}

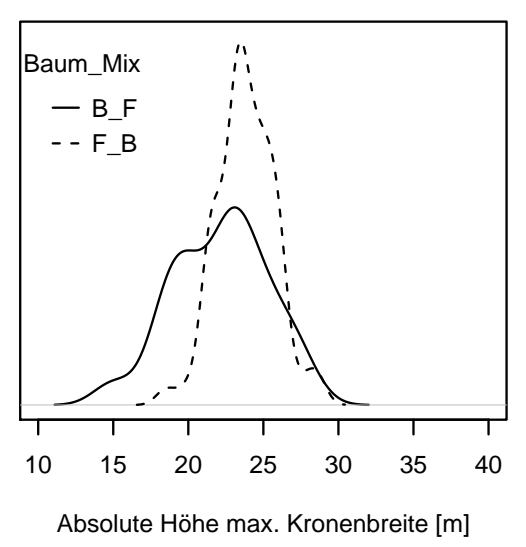

Abbildung 20: Höhe der maximalen Kronenbreiten in den aufgenommenen Versuchsflächen des GRK2300 als Wahrscheinlichkeitsdichte 


\subsubsection{Vergleich der horizontalen Kronenausdehnung}
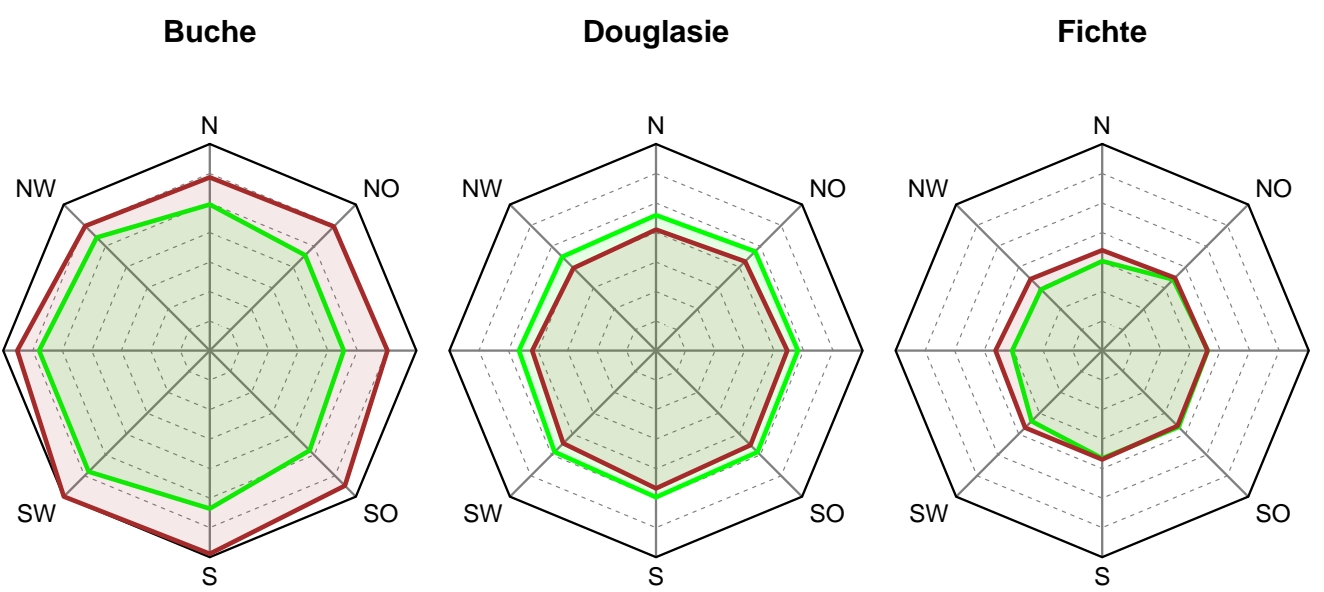

Abbildung 21: Gemittelte relative Kronenradien der Baumarten Buche, Douglasie und Fichte in den acht Himmelsrichtungen aufgeteilt nach Rein(grün)- und Mischbestand(rot).

Diese Auwertung beruht auf den Daten der NW-FVA und GRK2300, wie in Abschnitt 3.1.1 beschrieben. Die Kronenradien in Abbildung 21 wurden jeweils durch den BHD des zugehörigen Baumes geteilt, um ein relatives Maß zu erhalten und die Bäume miteinander vergleichen zu können. In dieser Grafik wird nochmals der Unterschied zwischen Rein- und Mischbestand deutlich und zudem ist hier auch die Information enthalten, ob es eine Asymmetrie der Kronen gibt. Die Buchen haben mit ihren größeren Kronenradien auch eine eher asymmetrische Krone im Vergleich mit Douglasien und Fichten. Diese ist im Mittel weiter in die südwestliche Himmelsrichtung ausgedehnt. Vorhanden ist dieser Effekt in Rein- und Mischbestand, wobei im Reinbestand eine verstärkte Ausdehnung in Richtung Westen zu erkennen ist. Bei der Douglasie und Fichte ist der gleiche Effekt weniger stark zu erkennen, da die Kronen weniger groß sind. Jedoch zeigen auch diese eine gewisse Asymmetrie bei der Kronenausdehnung. 


\subsubsection{Asymmetrie der Kronen}

Um die Untersuchung der Kronenausdehnung in Abschnitt 3.1.3 zu vertiefen, wurde ermittelt, inwieweit die Kronenradien von einer kreisrunden Krone abweichen. Hierfür wurde eine zirkuläre Normalverteilung („,von Mises“-Verteilung) der Kronenradien angepasst (gefittet) und die Parameter $\kappa$ und $\mu$ der Verteilung ermittelt. Bei dieser Verteilung wäre ein Baum mit kreisrunder Krone eine Gerade mit der Steigung 0. Je ovaler die Baumkrone ist, desto steiler verläuft die Kurve der Wahrscheinlichkeitsdichtefunktion. $\kappa$ ist der Ausdruck für diese Steigung und besagt, dass die Baumkrone bei höherem Wert ovaler ist. $\mu$ ist der Erwartungswert der Wahrscheinlichkeitsdichtefunktion und besagt in diesem Fall, in welcher Himmelsrichtung (in Gon) die Krone am weitesten von der kreisrunden Form abweicht. 
B_B

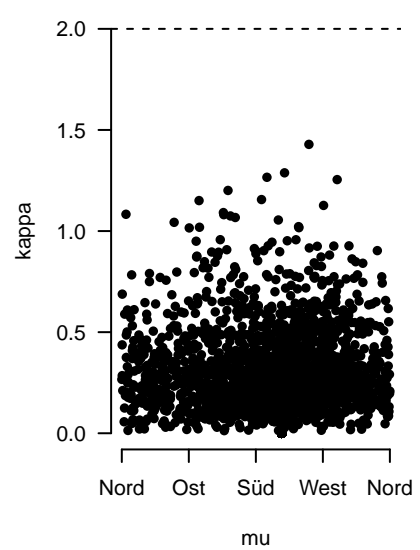

$\mathrm{mu}$

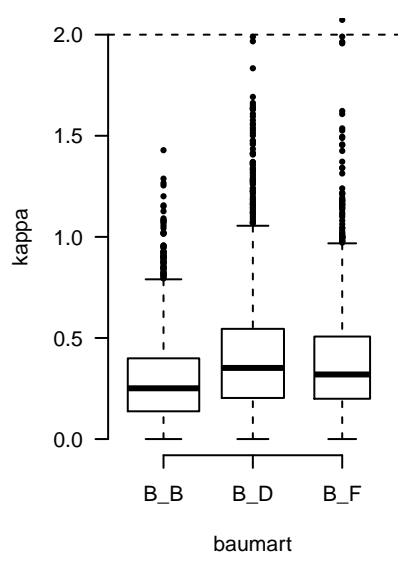

B_D

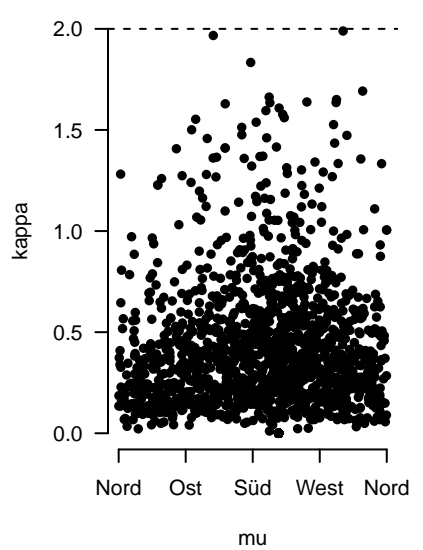

$\mathrm{mu}$

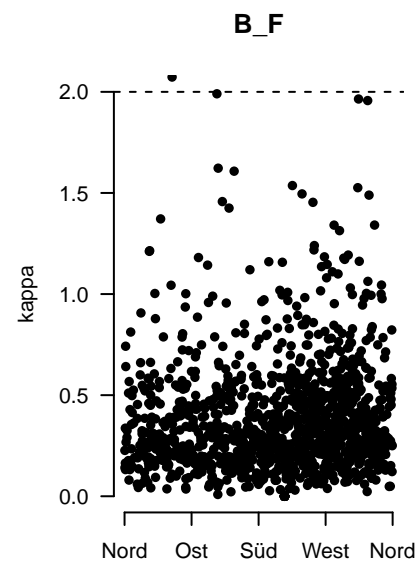

$\mathrm{mu}$

Abbildung 22: Ovalität der Buchenkronen in Reinbestand (B_B, $\mathrm{n}=1547$ ) und Mischbestand (B_D, $\mathrm{n}=1408 ; \mathrm{B} \_\mathrm{F}, \mathrm{n}=1122$ ) - Erwartungswert $\mu$ (Himmelsrichtung) und inverse Varianz $\kappa$ (Größe der Abweichung von kreisrunder Kronenform)

Die Ovalität der Buchenkronen ist in Mischung mit Douglasie oder Fichte größer als im Reinbestand. Die Boxplots zeigen die Verteilung der $\kappa$-Werte nochmals. Der Median der Mischungen mit den Koniferen liegt hier höher als beim Buchenreinbestand. Die Schwankungsbreite der $\kappa$-Werte ist in Mischung auch am höchsten. 
D_D

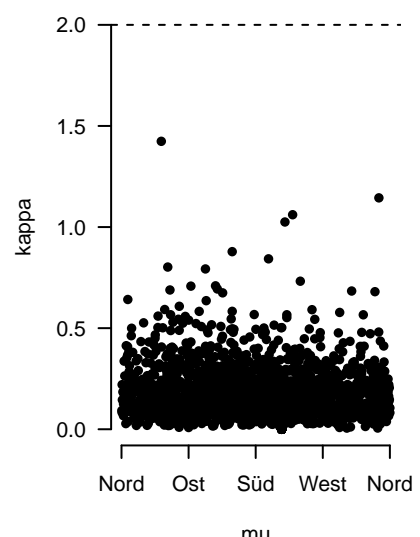

$\mathrm{mu}$

F_F

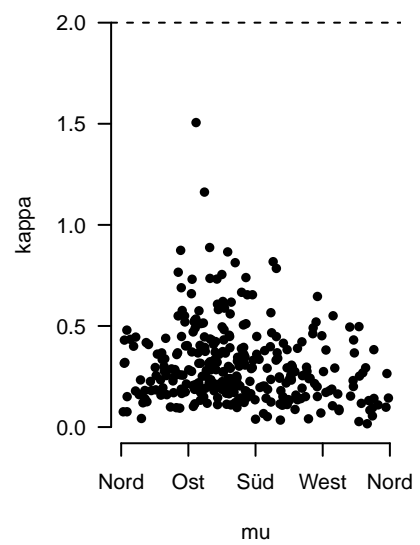

D_B
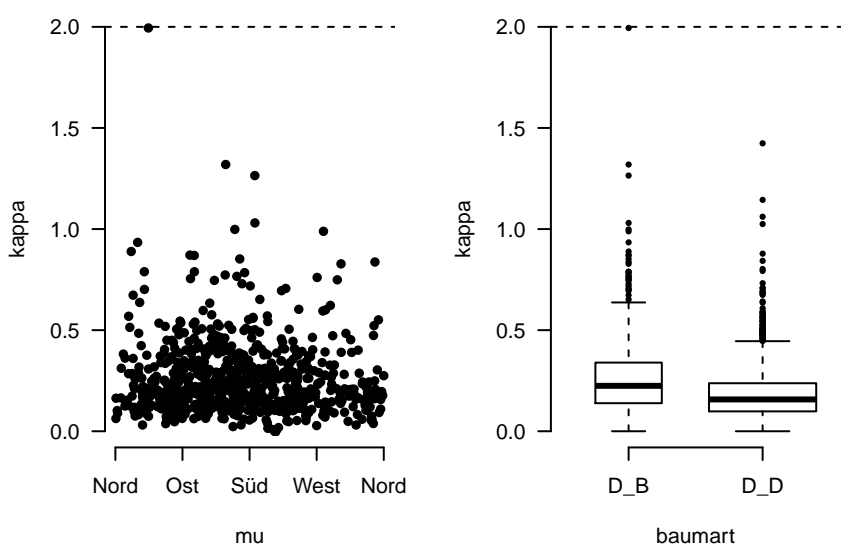

F_B
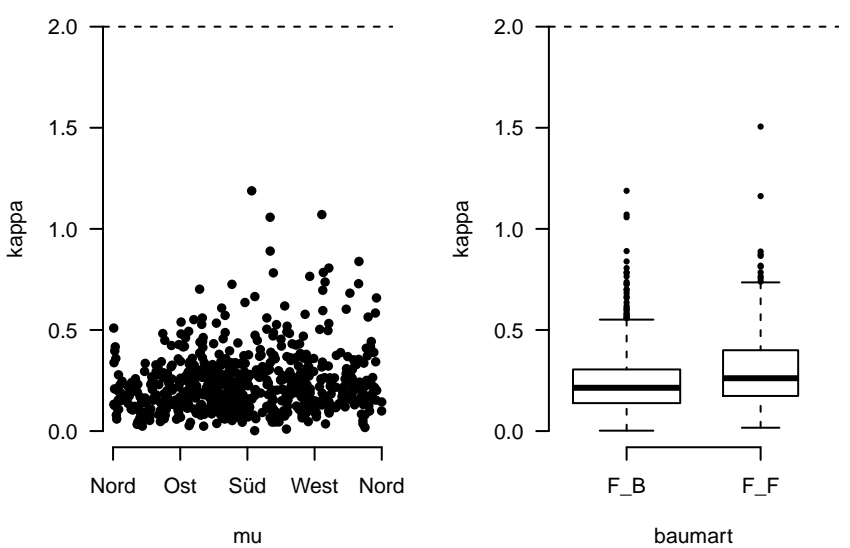

Abbildung 23: Ovalität der Baumkronen von Douglasie und Fichte in Reinbestand (D_D n $\left.=1727 ; \mathrm{F} \_\mathrm{F} \mathrm{n}=445\right)$ und Mischung mit Buche (D_B n $=787$; F_B n $=\overline{721})-$ Erwartungswert $\mu$ (Himmelsrichtung) und inverse Varianz $\kappa$ (Größe der Abweichung von kreisrunder Kronenform)

In Abbildung 23 ist das Ovalitätsmaß $\kappa$ für die Koniferen in Rein- und Mischbestand dargestellt. Im Gegensatz zu Abbildung 22 ist hier jedoch in Mischung keine höhere Ovalität zu erkennen. Bei der Douglasie ist hingegen sogar eine größere Ovalität im Reinbestand vorzufinden. Die $\kappa$-Werte haben bei der Douglasie in Mischung zudem eine geringere Schwankungsbreite als im Reinbestand. 
BU (B_D)

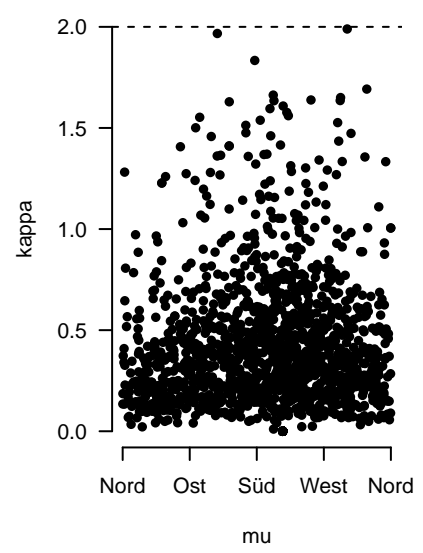

BU (B_F)

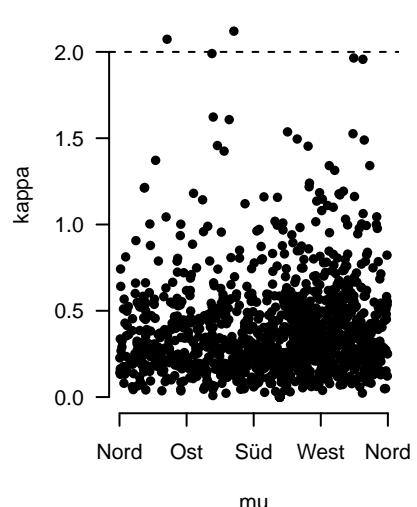

DGL (D_B)

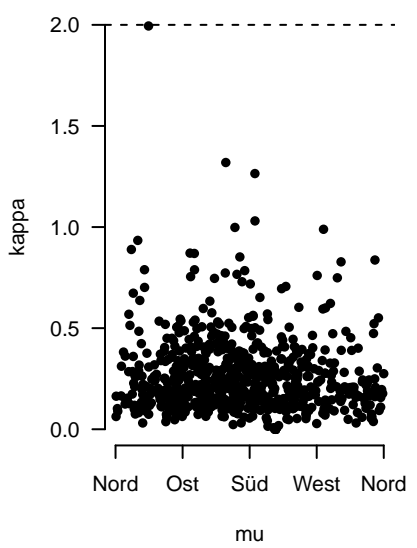

FI (F_B)
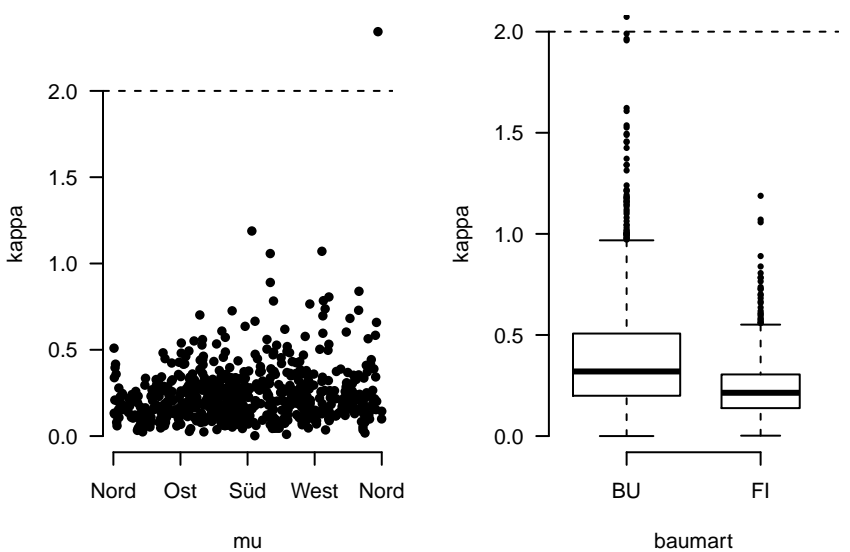

Abbildung 24: Ovalität der Baumkronen im Mischbestand (B_D $(\mathrm{n}=1408)$; D_B $(\mathrm{n}=787)$ und B_F $\left.(\mathrm{n}=1122) ; \mathrm{F} \_\mathrm{B}(\mathrm{n}=721)\right)$ - Erwartungswert $\mu$ (Himmelsrichtung) und inverse Varianz $\kappa$ (Größe der Abweichung von kreisrunder Kronenform)

In Abbildung 24 ist zu erkennen, dass die Buchen eine ovalere Baumkrone haben, als die Douglasienkronen. Der Median der $\kappa$-Werte bei den Buchen ist höher, wie in den nebenstehenden Boxplots zu erkennen ist. Man kann außerdem eine Verdichtung der Punktwolke in südlicher Richtung erkennen, was der südlichen Ausrichtung der Buchenkronen entspricht. 

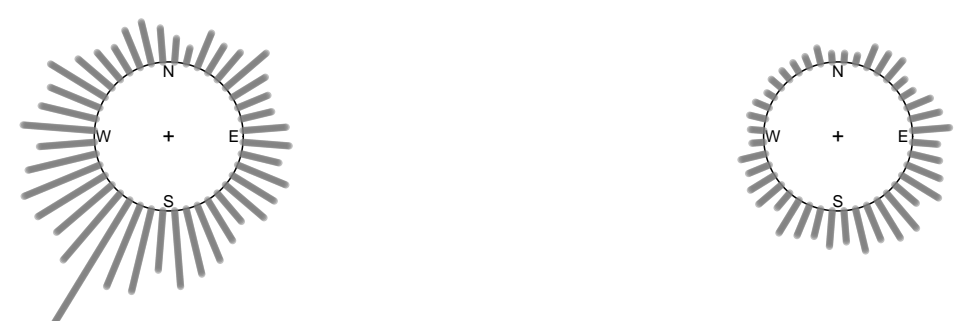

Buche (Mischung B_F)

Fichte (Mischung F_B)
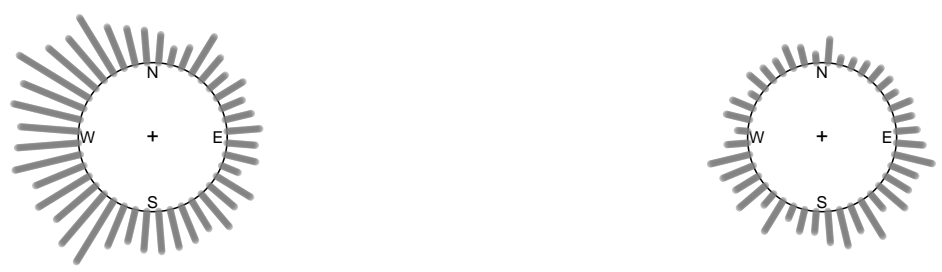

Abbildung 25: Ovalität der Baumkronen - Darstellung des Erwartungswerts $\mu$ zeigt die unterschiedliche Ausrichtung der Baumkronen

Abbildung 25 zeigt die aufsummierten Erwartungswerte $[\kappa]$ der an die Kronenradien gefitteten ,von Mises“-Verteilung über die entspechenden Himmelsrichtungen $[\mu]$ in den Mischbeständen Buche-Douglasie und Buche-Fichte für jeweils beide Baumarten ergänzend zu Abbildung 24. Der Erwartungswert ist bei den Buchen häufiger im Süd-Westen in Mischung mit Douglasie und im Westen bei Mischung mit Fichte. Bei Douglasie und Fichte in Mischung mit Buche häufiger im Süd-Osten. Die Ergebnisse zeigen ein ähnliches Muster wie die gemessenen und gemittelten Kronenradien in Abbildung 21. 


\subsection{Kronenprofile in Rein- und Mischbstand}

\subsubsection{Kronenprofildaten}
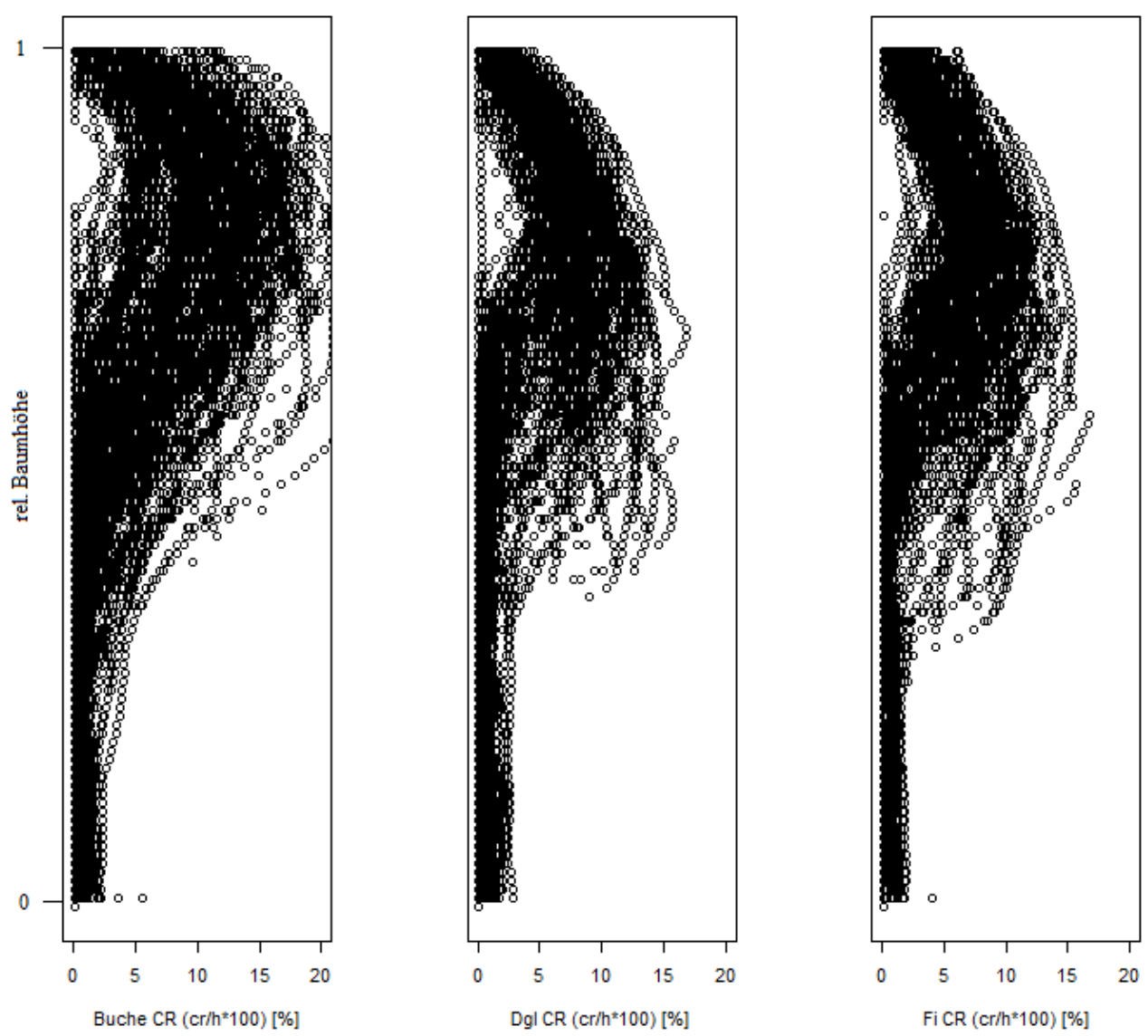

Abbildung 26: Kronenprofile als Punktdaten aller Kronenfotos. Die Baumlänge wurde auf 1 skaliert und die Kronenbreite ist der prozentuale Anteil an der Baumlänge eines jeden Baumes.

Die Auswertung der Kronenfotos (siehe Abschnitt 2.4) ergibt die in Abbildung 26 dargestellten Kronenprofile. Die Daten der Kronenfotos und alle hierauf beruhenden Auswertungsschritte stammen ausschließlich aus den acht Quintetts des GRK2300. Die Punktdaten zeigen das relative Kronenprofil der Bäume an, da die Kronenbreite den prozentualen Teil der Baumhöhe darstellt. Sie zeigen unter- 
schiedliche Kronenformen für jede der drei Baumarten.

Bei der Buche ist ein rundes Kronenprofil vorhanden, während es bei der Douglasie und Fichte eher pfeilförmig ist. Es ist außerdem sichtbar, dass die Buchenkronen eine höhere Streuung der Punkte haben. Dies gilt für die Kronenbreite, aber auch für die Kronenlänge. Die Punkte in den Kronen der Koniferen sind hingegen dichter und weniger variabel.

Zum direkten Vergleich wurden die Punktdaten der Baumarten in Abbildung 27 jeweils gemittelt. Der Unterschied der Kronenprofile von Buche zu den beiden Koniferen ist hier noch deutlicher zu erkennen. Die Form der Douglasie und Fichte ist fast identisch, allerdings wird durch die Relativierung der Punktwerte die absolute Baumgröße nicht abgebildet.

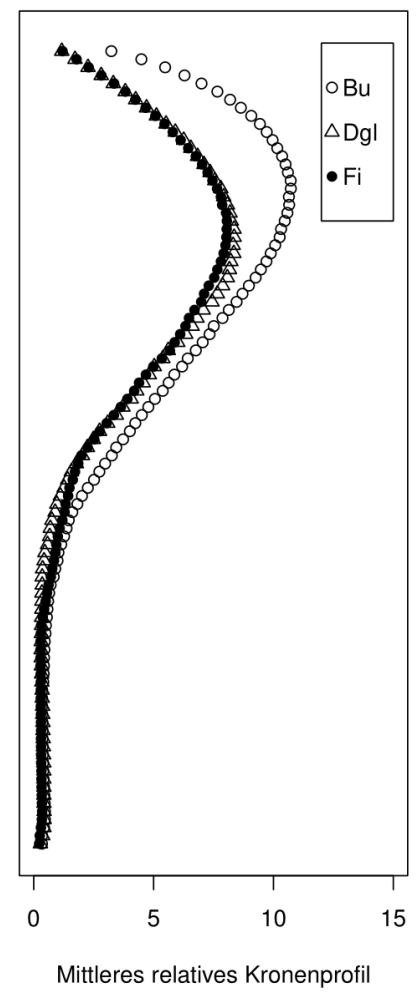

\subsubsection{Modellansatz}

In Anlehnung an Überlegungen zu Einflüssen von Nachbarbäumen auf die vorhandene Kronenform eines Bezugsbaumes in Buchen-Douglasien-FichtenMischbeständen wurden für die Herleitung einer Kronenformfunktion in Form der Weibull-Dichtefunktion drei Parameter -- shape $\gamma$, scale $\delta$ und fac $\xi$-- geschätzt.

Zur Notation der Modellierung werden mit Hilfe der Indikatorfunktion:

Abbildung 27: Mittlere relative Kronenprofile von Buche, Douglasie und Fichte. Skalierung Baumlänge auf 1. Kronenbreite entspricht dem prozentuale Anteil der Baumlänge.

$$
\mathrm{I}_{\{j=l\}}= \begin{cases}1, & \text { wenn } j=l \\ 0, & \text { sonst. }\end{cases}
$$

folgende Dummy-Variablen definiert: 


$$
\begin{aligned}
\operatorname{mix}_{\mathrm{BD}, i} & =\mathrm{I}_{\left\{\text {mix }_{i}=\text { Buche-Douglasie }\right\}} \\
\operatorname{mix}_{\mathrm{BF}, i} & =\mathrm{I}_{\left\{\text {mix }_{i}=\text { Buche-Fichte }\right\}} \\
\operatorname{mix}_{\mathrm{DB}, i} & =\mathrm{I}_{\left\{\text {mix }_{i}=\text { Douglasie-Buche }\right\}} \\
\operatorname{mix}_{\mathrm{DD}, i} & =\mathrm{I}_{\left\{\operatorname{mix}_{i}=\text { Douglasie-Douglasie }\right\}} \\
\operatorname{mix}_{\mathrm{FB}, i} & =\mathrm{I}_{\left\{\text {mix }_{i}=\text { Fichte-Buche }\right\}} \\
\operatorname{mix}_{\mathrm{FF}, i} & =\mathrm{I}_{\left\{\text {mix }_{i}=\text { Fichte-Fichte }\right\}}
\end{aligned}
$$

Eine solche Hilfsgröße wird nicht für die Kombination mix $\operatorname{BB}, i=$ Buche-Buche definiert, da diese in der Modellierung als Referenz-Kategorie verwendet wird, um das Modell identifizierbar zu halten (Fahrmeir et al., 2007). Die drei Zielvariablen wurden im Vorfeld der Modellierung Logarithmus-transformiert um bei einer Modellierung über die Annahme einer bedingten Normalverteilung für diese Größen die Eigenschaft, dass es sich bei eben diesen Größen um positive stetig skalierte Zufallsvariablen handelt, beachten zu können. ${ }^{3}$

\footnotetext{
${ }^{3}$ Nach einer Rücktransformation der geschätzten bedingten Erwartungswerte -- oder sonstiger prädiktiver Größen -- mittels der Exponentialfunktion werden diese Größen garantiert größer 0 sein.
} 
Es gilt folgender strukturiert additiver Modellansatz mit variierenden Koeffizienten (Fahrmeir et al., 2007):

$$
\begin{aligned}
\log \left(\gamma_{i}\right) & =\beta_{0, \gamma}+\beta_{1, \gamma} \operatorname{mix}_{\mathrm{BD}, i}+\ldots+\beta_{6, \gamma} \operatorname{mix}_{\mathrm{FF}, i}+ \\
& +\sum_{j=1}^{p}\left(f_{x_{j} \mid \operatorname{mix}_{\mathrm{BD}, i}, \gamma}\left(x_{j, i}\right) \operatorname{mix}_{\mathrm{BD}, i}+\ldots+f_{x_{j} \mid \operatorname{mix}_{\mathrm{FF}, i}, \gamma}\left(x_{j, i}\right) \operatorname{mix}_{\mathrm{FF}, i}\right)+ \\
& +\varepsilon_{i} \\
\log \left(\delta_{i}\right) & =\beta_{0, \gamma}+\beta_{1, \gamma} \operatorname{mix}_{\mathrm{BD}, i}+\ldots+\beta_{6, \gamma} \operatorname{mix}_{\mathrm{FF}, i}+ \\
& +\sum_{j=1}^{p}\left(f_{x_{j} \mid \operatorname{mix}_{\mathrm{BD}, i, \gamma}}\left(x_{j, i}\right) \operatorname{mix}_{\mathrm{BD}, i}+\ldots+f_{x_{j} \mid \operatorname{mix}_{\mathrm{FF}, i, \gamma}}\left(x_{j, i}\right) \operatorname{mix}_{\mathrm{FF}, i}\right)+ \\
& +\varepsilon_{i} \\
& +\varepsilon_{i} \\
& +\sum_{j=1}^{p}\left(f_{x_{j} \mid \operatorname{mix}_{\mathrm{BD}, i}, \gamma}\left(x_{j, i}\right) \operatorname{mix}_{\mathrm{BD}, i}+\ldots+f_{x_{j} \mid \operatorname{mix}_{\mathrm{FF}, i}, \gamma}\left(x_{j, i}\right) \operatorname{mix}_{\mathrm{FF}, i}\right)+ \\
\log \left(\xi_{i}\right) & =\beta_{0, \gamma}+\beta_{1, \gamma} \operatorname{mix}_{\mathrm{BD}, i}+\ldots+\beta_{6, \gamma} \operatorname{mix}_{\mathrm{FF}, i}+ \\
&
\end{aligned}
$$


Tabelle 15: Tabelle mit Abkürzungen der Prädiktoren

\begin{tabular}{|c|c|c|}
\hline Abkürzung & Kürzel & Einflussgröße \\
\hline \hline$x_{1}$ & dist & Baumdistanz $[\mathrm{m}]$ \\
\hline$x_{2}$ & bhd_diff & Durchmesserunterschied (BHD/BHDnachbar) \\
\hline$x_{3}$ & h_diff & Höhendifferenz $[\mathrm{m}]$ \\
\hline$x_{4}$ & h & Baumhöhe $[\mathrm{m}]$ \\
\hline$x_{5}$ & bhd & BHD [mm] \\
\hline$x_{6}$ & ang & Winkel: Kronenansatz zu Nachbarbaumspitze \\
\hline
\end{tabular}

Die Effekte der unterschiedlichen Einflussgrößen variieren über die verschiedenen Baumartenkombinationen und zusätzlich bei den drei Modellen (shape $\gamma$, scale $\delta$, fac $\xi$ ), sodass eine Selektion der Einflussgrößen durchgeführt werden muss. Hierzu wird mittels mgcv: :gam (Wood, 2011) und der Option select=TRUE gearbeitet. Wenn select=TRUE an die gam-Funktion übergeben wird, kann diese aus dem R-Paket mgcv jedem Term einen zusätzliche Strafterm hinzufügen, sodass er auf Null reduziert werden kann. Dies bedeutet, dass die Schätzung der Glättungsparameter, die Teil der Anpassung ist, Terme vollständig aus dem Modell entfernen kann. Wenn der entsprechende Glättungsparameter auf Null geschätzt wird, hat der zusätzliche Strafterm keine Auswirkung. In Tabelle 16 werden die selektierten Modellkomponenten aufgeführt. 
Tabelle 16: Geschätzte Freiheitsgrade (edf) der einzelnen Modellkomponenten zur Feststellung der Signifikanz einzelner Einflussgrößen. „-“ bedeutet keinen Effekt der Einflussgröße auf die Zielvariable.

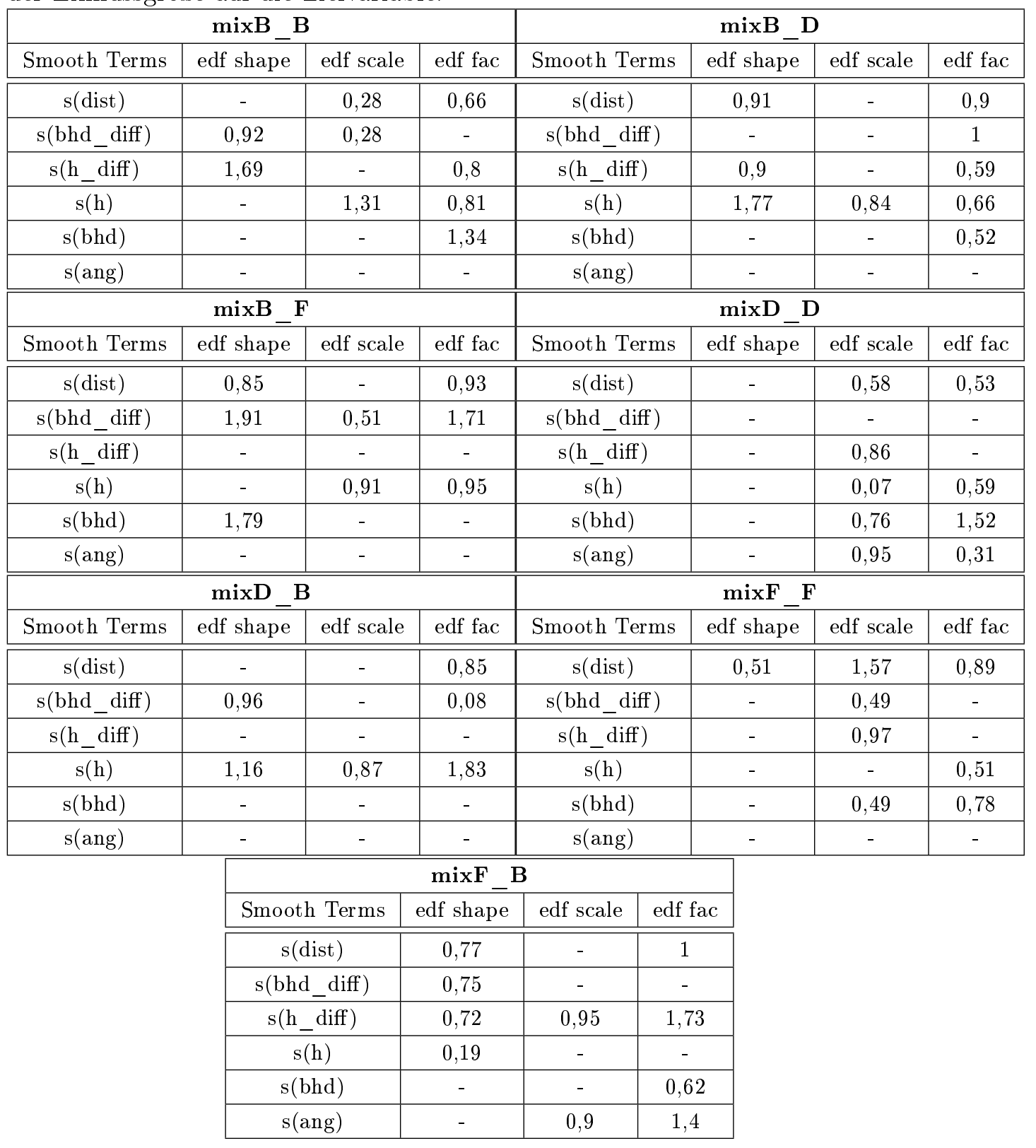


Die selektierten Modellkomponenten in Tabelle 16 wurden nach der Baumartenmischung aufgeteilt. Zu erkennen ist, dass für bestimmte Baumartenmischungen manche Modellkomponenten weniger Einfluss haben, als andere. Beispielsweise ist bei mixD_D kein Effekt der Einflussgrößen auf den Shape-Parameter vorhanden. Folglich wird die Kronenform der Douglasie neben einer weiteren Douglasie nicht durch diesen Parameter beeinflusst (mögliche Effekte von Shape und Scale Paramter in Abbildung 28), sondern nur durch die anderen beiden Parameter, da es bei dem Scale-Parameter und Fac-Parameter selektierte Einflussgrößen gibt. Der Fac-Parameter dient zur Multiplikation mit der Weibull-Dichtefunktion, um die Funktion auf reale Kronenbreiten in [m] aufzuspannen, da sich mit der Dichtefunktion sonst nur relative Kronenformen darstellen ließen (die Fläche unter der Dichtefunktion ergibt 1, sodass die Werte der Y-Achse ohne Multiplikator keine reellen Werte representieren (siehe Abbildung 29)).
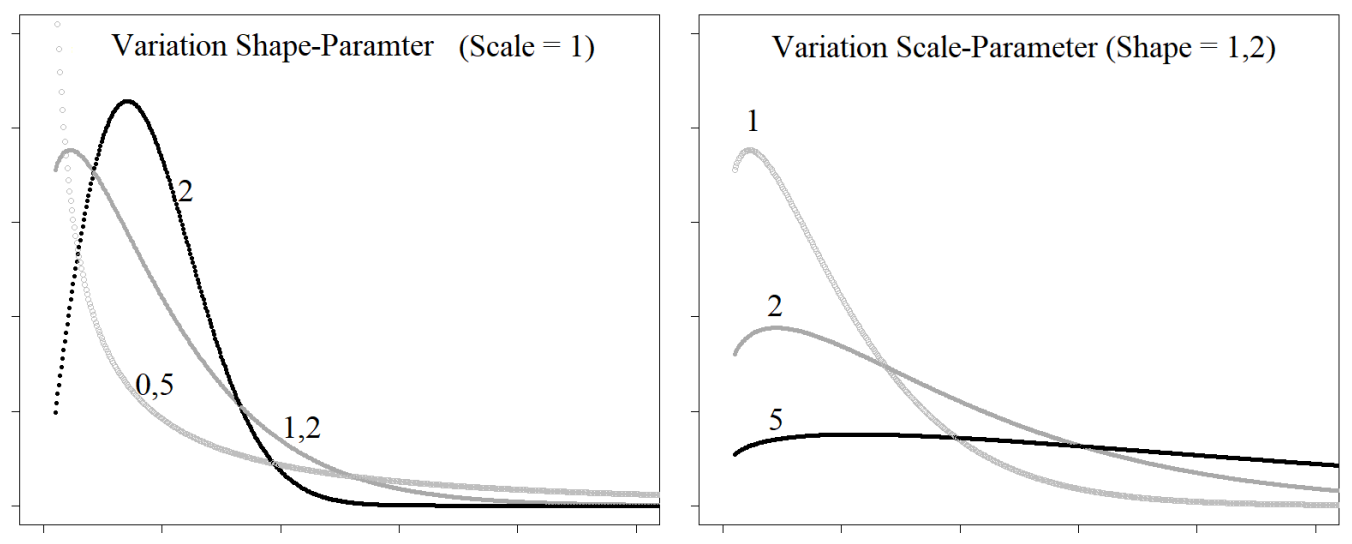

Abbildung 28: Effekte von unterschiedlichen Shape- und Scale-Parametern auf den Verlauf der Weibull-Funktion und somit auf die Kronenform 


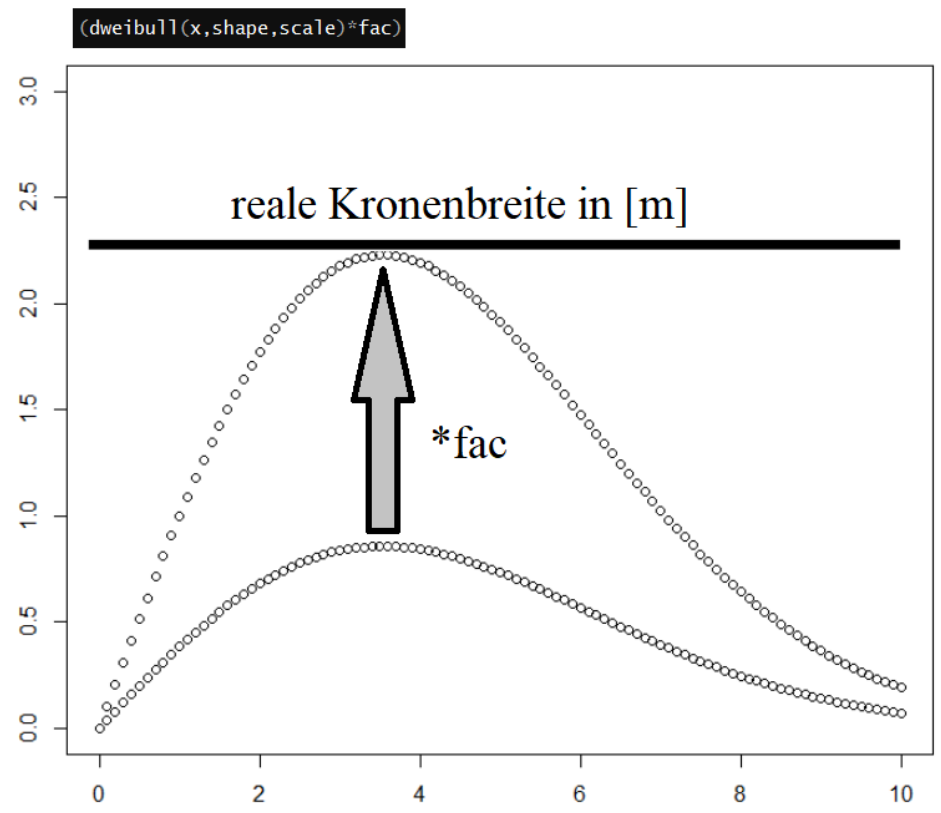

Abbildung 29: Veranschaulichung der Funktionsweise des Fac-Parameters zum Aufspannen der Weibull-Dichtefunktion 


\subsubsection{Reaktionsmuster auf veränderte Nachbarschaft}

Um die Reaktionsmuster der Baumkronen in verschiedenen Nachbarschaftssituationen zu veranschaulichen, wurden die Modellkronen mit varrierenden Prädiktorgrößen dargestellt. Die Effekte einzelner Prädiktoren werden sichtbar gemacht, indem nur ein Wert verändert und die daraus resultierende Kronenform dargestellt wird. Die Darstellung ist zweidimensional und dient ausschließlich der Modellveranschaulichung. Zur Bestandesdarstellung werden diese Modelle in einem dreidimensionalen Raum verwendet (2.8).
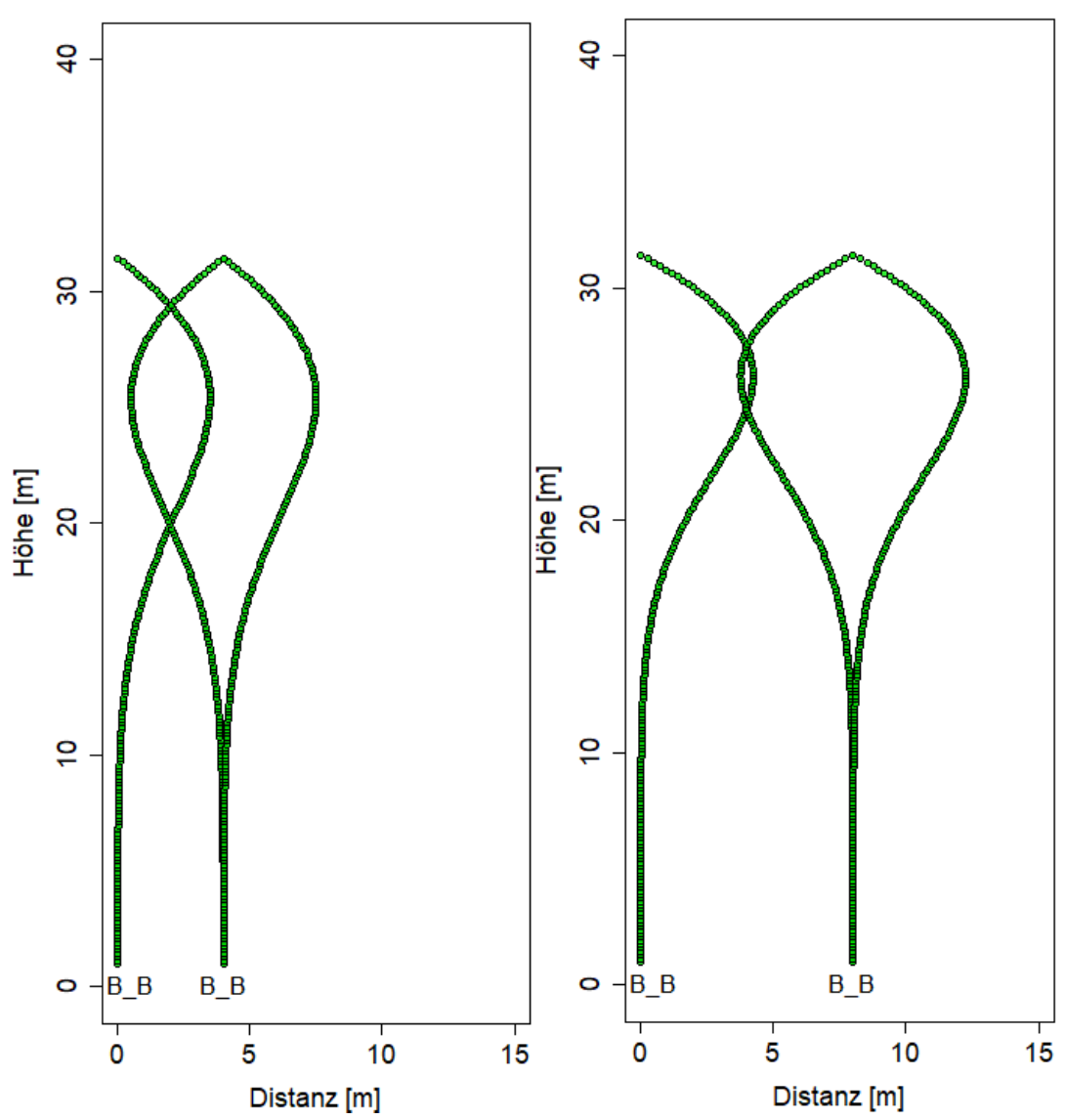

Abbildung 30: Reaktion der Kronenform bei Buche neben Buche, varriert wird die Distanz als Prädiktor (links: $4 \mathrm{~m}$; rechts $8 \mathrm{~m}$ ). BHD $50 \mathrm{~cm}$ bei beiden Bäumen.

Bei einer größeren Distanz der beiden Baumkronen haben diese größere Kronenradien. Dies wird in Abbildung 30 bei einem direkten Vergleich der beiden 
Situationen sichtbar. Bei einer Baumdistanz von 4 Metern hat die Kronen eine schmalere Form, zudem gibt es einen vergleichsweise großen Überschneidungsbereich, welcher einen potentiell gemeinsam genutzten Kronenraum kennzeichnet. In diesem Bereich kann es folglich zu einem Ineinanderwachsen der Kronen kommen. Die schmalere und spitzere Krone bei dichtem Stand kann als Reaktion auf die erhöhte Konkurrenz durch die Nachbarbuche interpretiert werden.
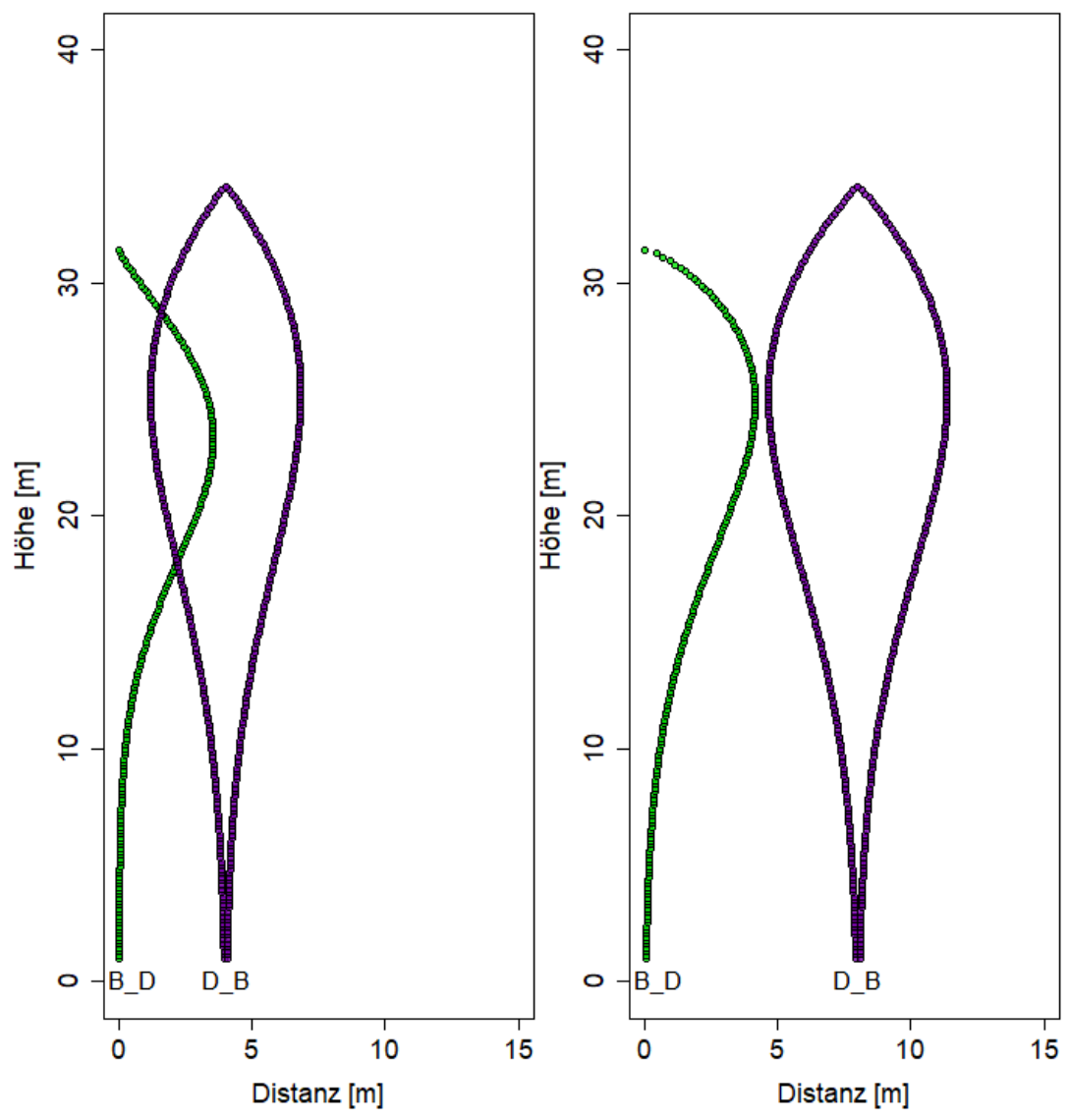

Abbildung 31: Reaktion der Kronenform bei Buche (grün) neben Douglasie (violett), variiert wird die Distanz als Prädiktor. BHD $50 \mathrm{~cm}$ bei beiden Bäumen.

Die Reaktion der Buchen- und Douglasienkronen in benachbartem Wuchs ist in Abbildung 31 veranschaulicht. Die Buchenkrone verändert ihre Form, wenn die Distanz zu der benachbarten Douglasie variiert wird. Bei vier Metern Abstand ist diese tropfenförmig, was zur Folge hat, dass die Buche mit ihrer Krone in die Dou- 
glasie hineinwächst und (relativ) am Stamm nach unten wandert. Die Douglasie ist bei gleichem BHD höher als die Buche und überragt diese. Bei der erhöhten Distanz von 8 Metern ist die Buchenkrone nicht mehr tropfenförmig, sondern wieder bauchig, ähnlich wie die Buchenkrone neben einer anderen Buche (Abbildung 30). Die Buchen- und Douglasienkronen haben hierbei keine Überschneidung mehr, was auch für eine eher normale Wuchsform spricht und bei konkurrenzarmen Standverhältnissen am ehesten zu erwarten ist. Die Douglasienkronen unterscheiden sich nur schwach in beiden Ausprägungen. Der Kronenradius ist bei höherer Distanz zur Buche etwas vergößert.
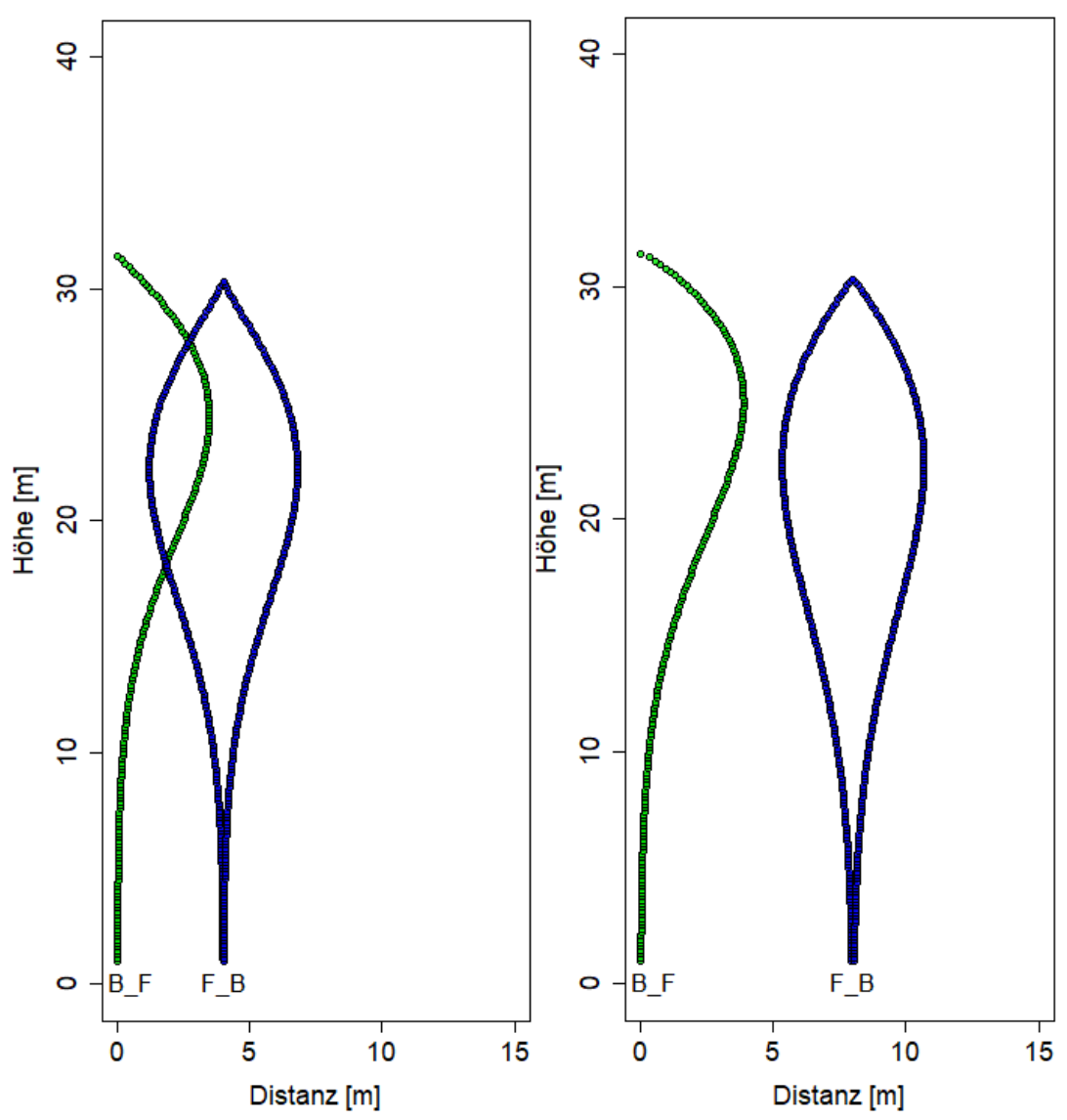

Abbildung 32: Reaktion der Kronenform bei Buche neben Fichte, variiert wird die Distanz als Prädiktor. BHD $50 \mathrm{~cm}$ bei beiden Bäumen.

Bei der Mischung von Buche und Fichte lassen sich am wenigsten Effekte des 
Kronenmodells feststellen. Hier bewirkt die Distanzänderung keine Veränderung der Kronenform bei Buche oder Fichte, wenn beide Bäume den gleichen BHD (hier $50 \mathrm{~cm}$ ) haben.
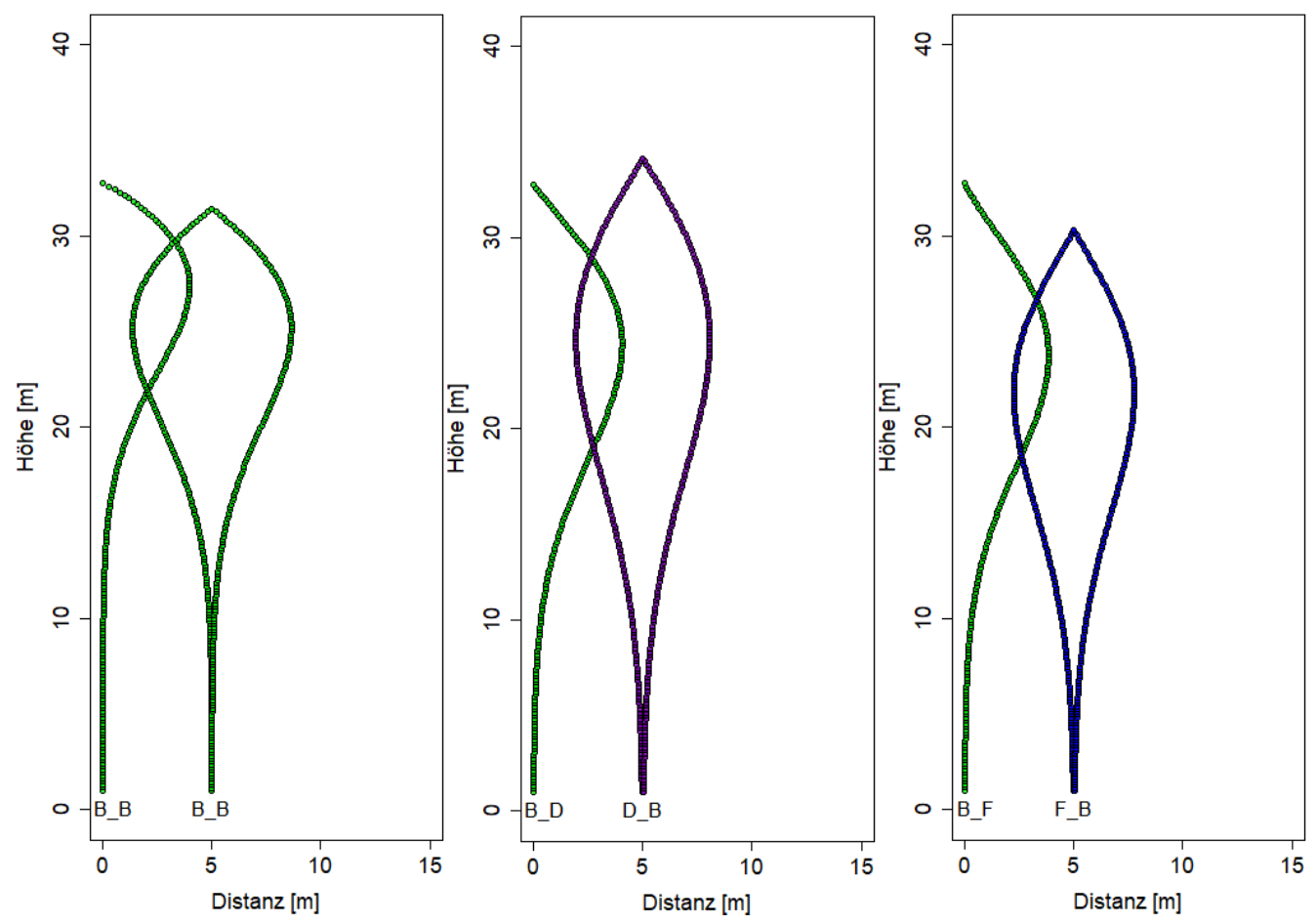

Abbildung 33: Reaktion der Kronenform, variiert wird die Art als Prädiktor. Distanz $5 \mathrm{~m}$; BHD linksW: $60 \mathrm{~cm}$; BHD rechts: $50 \mathrm{~cm}$.

In Abbildung 33 wird die Baumart variiert und gleichzeitig werden alle anderen Prädiktoren beibehalten. Dadurch wird der Unterschied in der Kronenform der Buche bei den verschiedenen Artkombinationen sichtbar. Die linke, größere Buche $(\mathrm{BHD}=60 \mathrm{~cm})$ hat neben einer weiteren, kleineren Buche $(\mathrm{BHD}=50 \mathrm{~cm})$ eine rundliche Kronenform. Im Gegensatz dazu ist sie neben einer Douglasie oder Fichte eher tropfenförmig. 

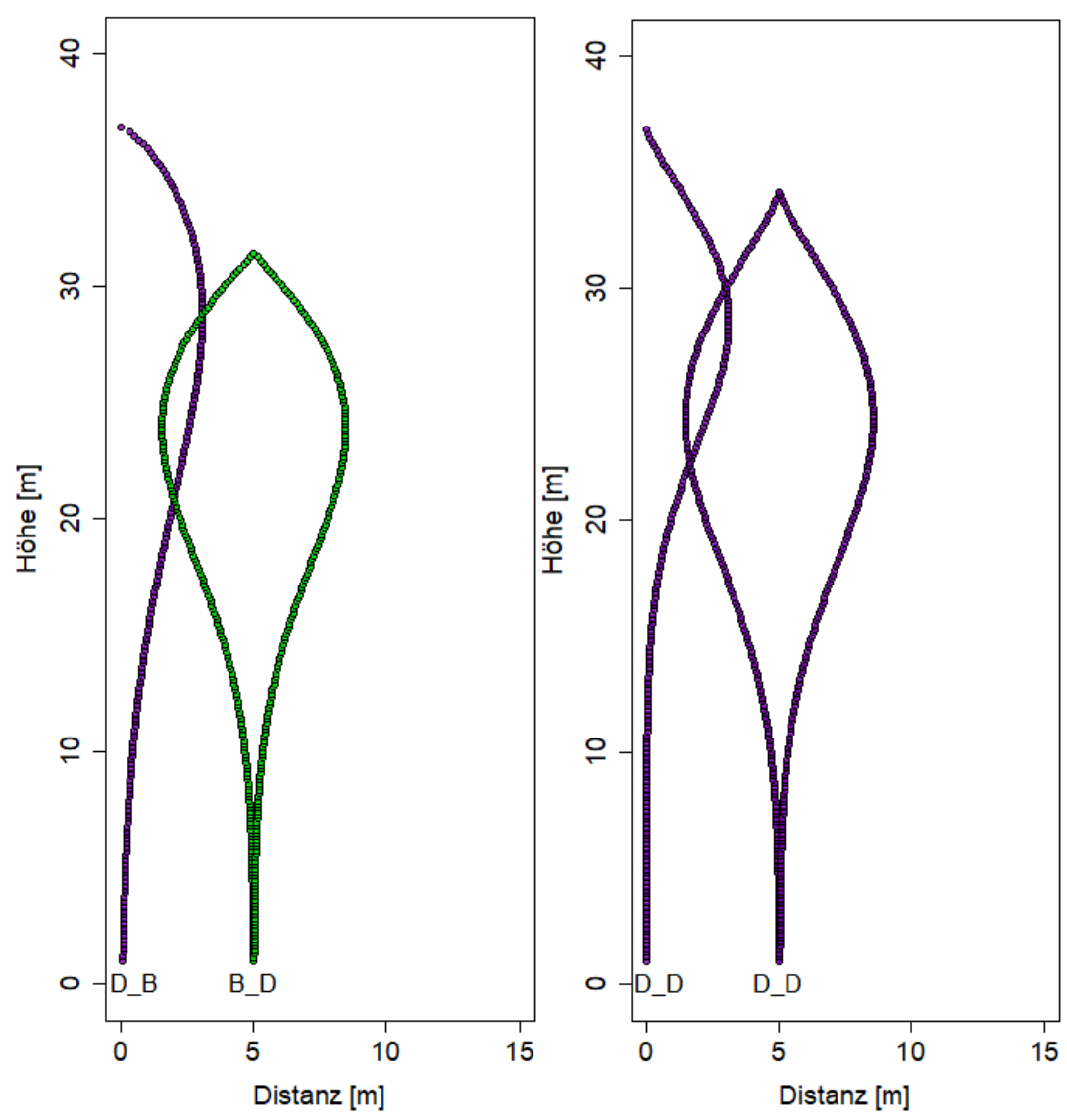

Abbildung 34: Reaktion der Kronenform. Vergleich: Douglasie neben Buche und Douglasie, variiert wird die Art als Prädiktor. Distanz 5 m; BHD linksW: 60 $\mathrm{cm}$; BHD rechts: $50 \mathrm{~cm}$.

Die Reaktion einer Douglasienkrone ist in Abbildung $34 \mathrm{zu}$ sehen. Die Krone hat neben einer kleineren Buche eine rundlichere Form, als wenn die Douglasie neben einer kleineren Douglasie steht. Die Douglasienkrone verschiebt sich, ähnlich wie bei der Buche neben Buche in Abbildung 33, etwas nach oben. 

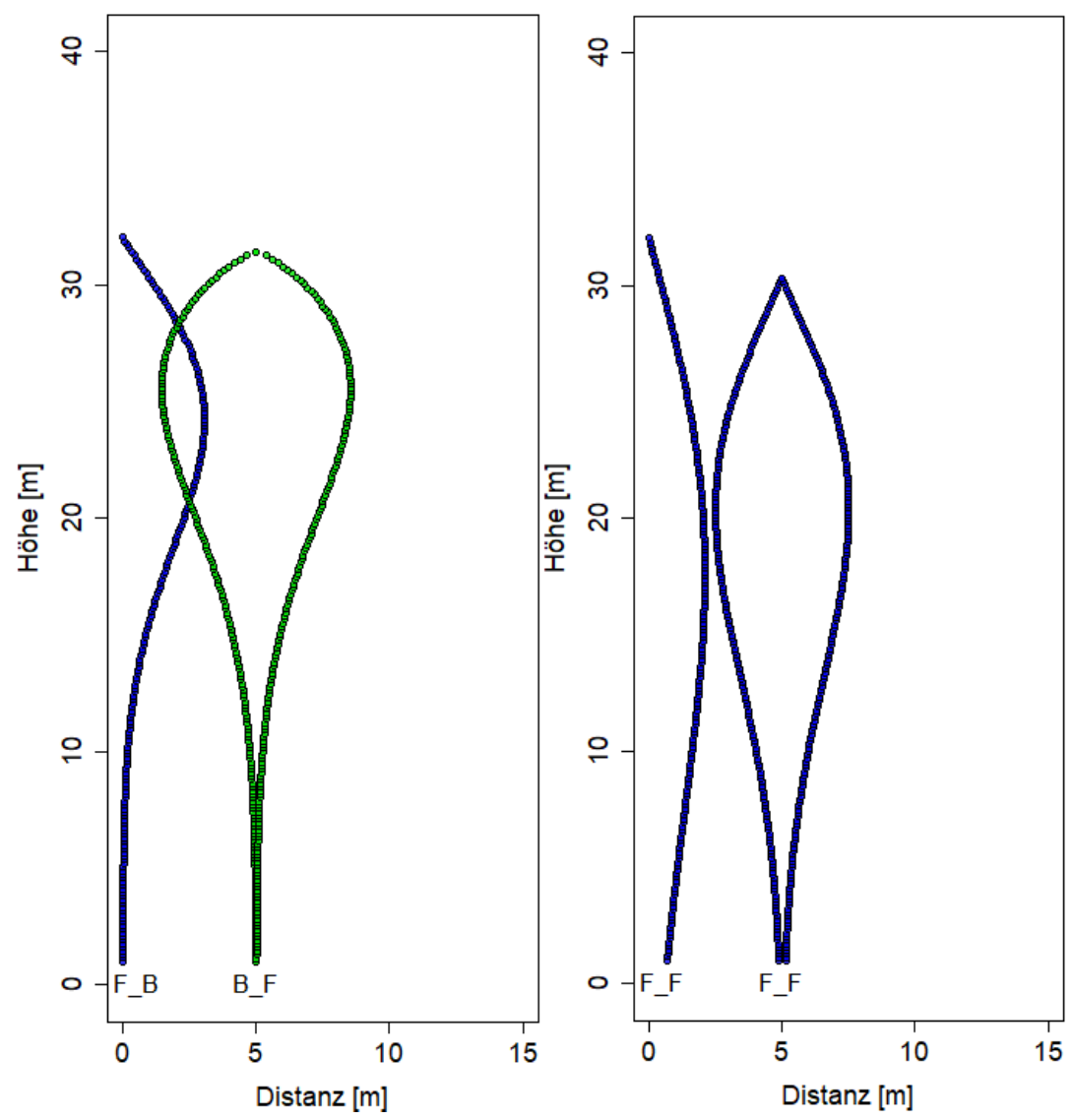

Abbildung 35: Reaktion der Kronenform. Vergleich: Fichte neben Fichte und Buche, variiert wird die Art als Prädiktor. Distanz 5 m; BHD links W: $60 \mathrm{~cm}$; BHD rechts: $50 \mathrm{~cm}$.

Bei der Kronenform der Fichte ist auffällig, dass diese neben einer weiteren Fichte sehr flach verläuft. Zudem ist die Kronenlänge bei dieser Kombination modellbedingt sehr groß und verläuft bis zum Stammfuß. Befindet sich die Fichte neben einer Buche, ist ein höherer Kronenansatz vorhanden, ihre Form wird runder und die Höhe der maximalen Kronenbreite verschiebt sich nach oben.

\subsubsection{Eingrenzung der Modellschätzung}

Für eine allgemeingültige Anwendbarkeit der Modelle, d.h. Kronenformschätzung für beliebige Bäume, ist es nötig, das Modell in bestimmten Datenbereichen zu begrenzen, um die Kronenform in ihrer Ausdehnung auch bei Extremfällen in bio- 
logisch plausiblen Rahmen zu halten. Grund für die Begrenzung ist die Datenlage mit hauptsächlich oberständigen Baumindividuen. Dies ist darauf zurückzuführen, dass die Versuchsflächen in durchforsteten Beständen ausgesucht wurden und außerdem für die Aufnahme der Kronenfotos eine Teilmenge der Einzelbäume aufgenommen wurde. Darüber hinaus wurden besonders kleine und unterständige Bäume nur selten erfasst, weil diesen für die primäre Kronenuntersuchung eine untergeordnete Rolle zugewiesen wurde. Schließlich haben die Versuchsflächen eine gewisse Altersspanne von 60 - 100 Jahren, sodass hier Jungwuchsphasen nicht vorhanden sind. Die in das Modell einfließenden Variablen werden beim Bestandesaufbau durch das Programm auf ihren Gültigkeitsbereich geprüft. Hierbei ist der BHD entscheidend, ob ein Baum durch die Modellkrone repräsentiert werden soll, oder ob diesem eine Ersatzkrone, beispielsweise eine symmetrische Kronenform, abhängig von dem Kronenradius und der Kronenlänge (Pretzsch, 2009), aufgesetzt werden soll. Fällt ein BHD-Wert eines Baumes unter das 95\% Quantil der in das Modell eingeflossenen BHD-Werte, so wird diesem automatisch eine Ersatzkrone aufgesetzt. Weitere Begrenzungen der Modellwerte sind bei dem geschätzten Shape-Parameter eingebaut. Da dieser bei Werten $<=1$ zu unendlichen Kronenbreiten führt, wurde der Minimalwert auf 1,4 gesetzt. Ein geschätzter Shape-Parameter kann somit nur minimal den Wert 1,4 haben. Für den Maximalwert wurden keine unplausiblen Schätzungen festgestellt, welches eine Begrenzung nicht nötig macht. Bei dem ,Scale“ Parameter wurde der Minimalwert aus dem selben Grund auf 7 beschränkt. Für den „Fac“ Parameter waren keine Begrenzungen der Schätzung erforderlich.

\subsection{Modelleffekte auf Bestandesebene}

Die unterschiedlichen Reaktionsmuster der Krone auf Seitendruck durch inner- und zwischenartliche Konkurrenz wurden in Abbildung 30 bis Abbildung 35 dargestellt. Bei Anwendung dieser Reaktionsmuster in realen oder simulierten Bestandeskonstellationen können diese Effekte zu einer veränderten Kronenraumnutzung im 
Vergleich zu einem nachbarschaftsunabhängigen Kronenmodell führen. Der folgendende hier dargestellte Schritt ist die Anwendung des Modells auf Bestandesebene. Die Ergebnisse beruhen auf den in Abschnitt 2.12 getroffenen Einschränkungen für den Vergleich von Rein- und Mischbestand, das heißt, dass nur simulierte Mischbestände mit den entsprechenden Reinbeständen und anteilsmäßiger Grundfläche sowie gleichem Alter verglichen werden. Es werden die beleuchtete Kronenmantelfläche und das Kronenvolumen für Bestände mit der Modellkrone sowie mit einer symmetrischen nachbarschaftsunabhängigen Krone gegenübergestellt. In den anschließend vorgestellten Ergebnissen werden folgende Begriffe für die verschiedenen Kronenmodelle verwendet:

1. Symmetrisches Kronenmodell - Das symmetrische Kronenmodell beruht auf geometrischen Kronenmodellen (Pretzsch, 1992b) und wird im Waldwachstumssimulator SILVA der TU-München (Pretzsch et al., 2002) verwendet. Dies benötigt als Eingangvariablen Kronenlänge, maxmialer Kronenradius und Baumart. Es verwendet zwei Funktionen, eine für die Lichtkrone und eine für die Schattenkrone. Für verschiedene Baumarten sind unterschiedliche Parameter für die Funktionen definiert, welche die Kronenform artspezifisch verändert.

2. Asymmetrisches Kronenmodell - Das asymmetrische Kronenmodell ist das im Rahmen dieser Arbeit erstellte Kronenmodell, welches in 3.2.2 vorgestellt wurde.

Die beleuchtete Kronenmantelfläche in Abbildung 36 und Abbildung 37 ist in den simulierten (mit anteilsmäßiger Grundfläche der Reinbestände, je nach Mischungsanteil) Mischbeständen größer als in den dazugehörigen Reinbeständen. Auffällig ist, dass die Buche in den Mischbeständen eine größere beleuchtete Kronenoberfläche aufweist. Die Koniferen profitieren auch von einer Mischung - die beleuchtete Kronenfläche fällt bei diesen allerdings unterschiedlich aus. Die Douglasie in Abbildung 36 hat eine Vergrößerung in ähnlichem Maße wie die Buche, 
wohingegen die Fichte in Mischung mit Buche (Abbildung 37) in allen Mischungsvariationen fast gänzlich auf der 1.0 Linie bleibt und somit die selbe beleuchtete Kronenmantelfläche aufweist, wie in den dazugehörigen Reinbeständen.

Der Vergleich der verwendeten Kronenmodelle (Abbildung 36 und Abbildung 37) zeigt, dass bei den Koniferen die Douglasie im symmetrischen Kronenmodell eine größere beleuchtete Kronenmantelfläche besitzt. Jedoch ist dieser Effekt nicht im asymmetrischen Kronenmodell vorhanden. Dort hat die Douglasie einen um die 1.0 schwankenden Wert, welcher im Median teilweise sogar unter 1.0 liegt. Trotz dieser geringeren Fläche bei den Douglasien hat der Mischbestand im Ganzen ähnlich hohe beleuchtete Kronenmantelflächen. Die Buche gleicht den geringeren Wert der Douglasie umso stärker aus. Bei den Fichten ist der Effekt ähnlich wie bei den Douglasien, wobei hier die Gesamtoberfläche beider Baumarten im asymmetrischen Modell geringer ausfällt als beim symmetrischen. 

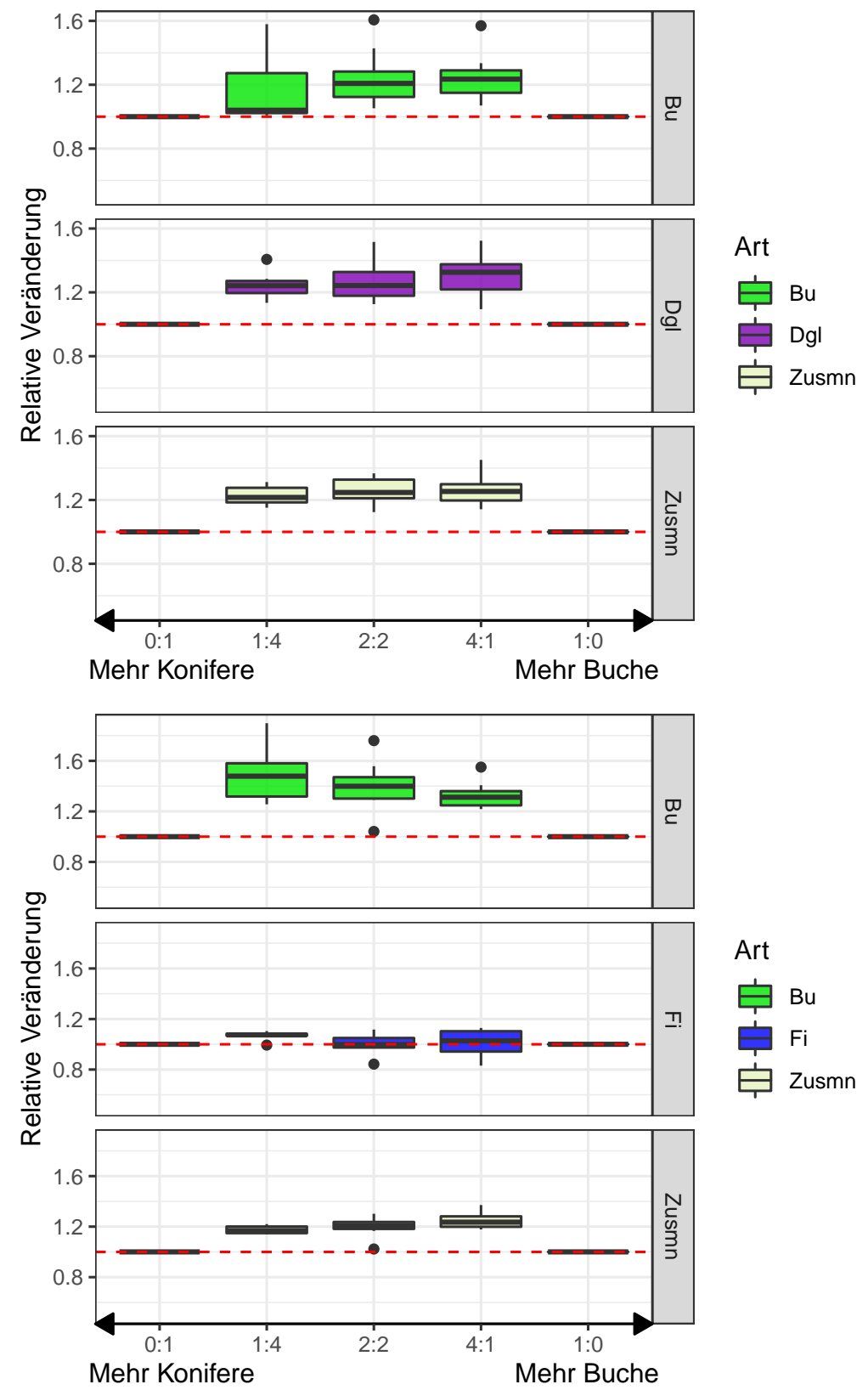

Abbildung 36: Beleuchtete Kronenmantelfläche mit symmetrischer Kronenform. Relative Veränderung bei generierten Mischbeständen mit $\mathrm{Bu}$ che/Konifere im Vergleich zu Reinbeständen, welche auf der roten Linie mit 1,0 angesiedelt sind. Die Baumarten haben in den generierten Mischbeständen eine variierende $\mathrm{G} /$ ha $(25 \%, 50 \%, 75 \%)$, jedoch immer die selbe Bonität und Alter wie die zugehörigen Reinbestände. 


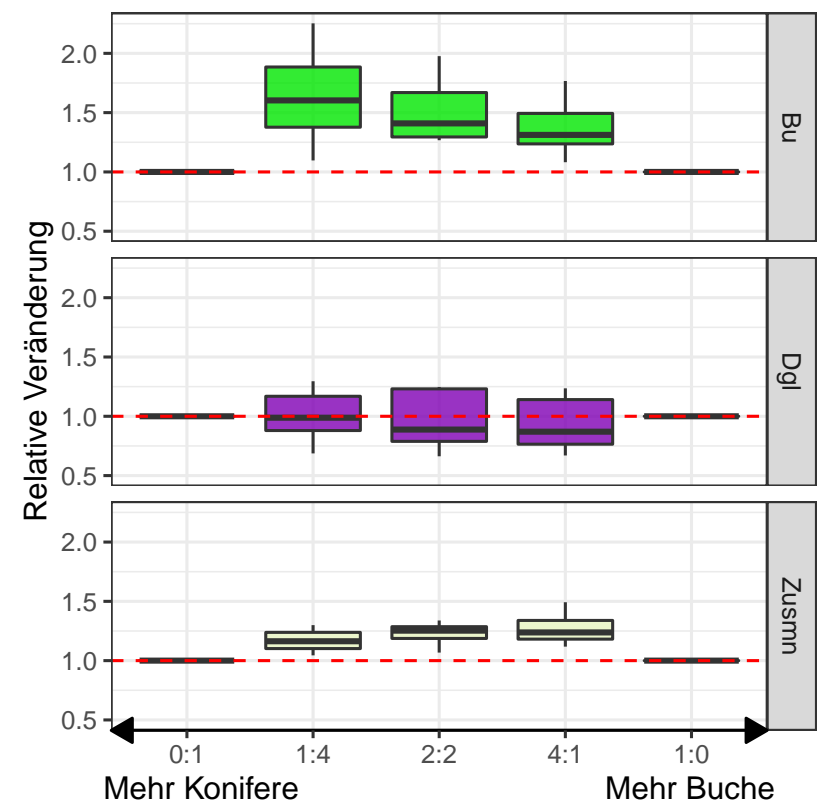

Art

E Bu

E Dgl

追 Zusmn

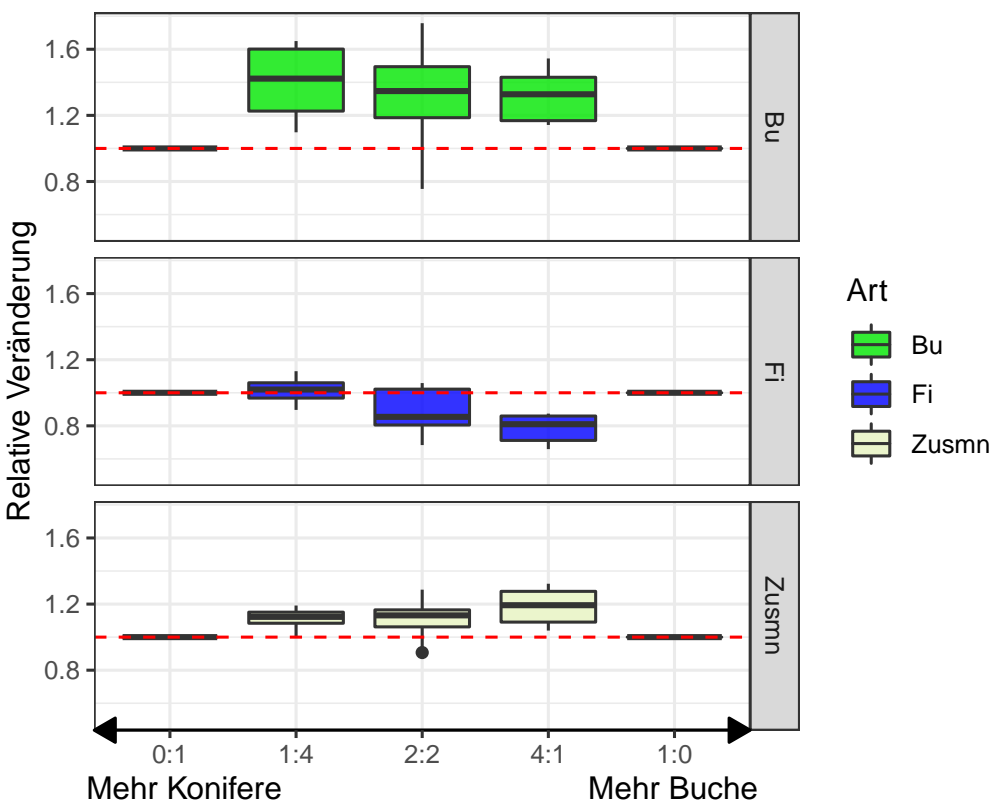

Abbildung 37: Beleuchtete Kronenmantelfläche mit asymmetrischer Kronenform aus Kronenmodell. Relative Veränderung bei generierten Mischbeständen mit Buche/Konifere im Vergleich zu Reinbeständen, welche auf der roten Linie mit 1,0 angesiedelt sind. Die Baumarten haben in den generierten Mischbeständen eine variierende $\mathrm{G} / \mathrm{ha}(25 \%, 50 \%, 75 \%)$, jedoch immer die selbe Bonität und Alter wie die zugehörigen Reinbestände. 
Das Kronenvolumen steht in Relation mit der Kronenmantelfläche. Bei der beleuchteten Kronenmantelfläche sollte diese Relation auch noch gegeben sein, allerdings nicht mehr so stark, da sich bei veränderter Bestandesstruktur durch Überschattungen Differenzen in der beleuchteten Kronenoberfläche und der dazugehörigen Krone im Bestand ergeben können. Die Ergebnisse zeigen, dass das Kronenvolumen im Mischbestand in allen Kombinationen leicht über 1 oder auf 1.0 ist. Ein positiver Effekt ist jedoch hier fast ausschließlich auf die Buche zurückzuführen. Lediglich in Abbildung 38 hat die Douglasie ein etwas erhöhtes Volumen, jedoch viel geringer, als der positive Effekt bei der beleuchteten Kronenmantelfläche der Douglasien.

Der Vergleich der verwendeten Kronenmodelle mit Auswertung des Kronenvolumens zeigt, dass beide Varianten ein höheres Kronenvolumen im Mischbestand aufweisen. Dabei hat das asymmetrische Kronenmodell den größten positiven Effekt bei einem geringen Buchenanteil. In Abbildung 39 ist ein Abfall der Kronenvolumina der Buchen und Fichten bei steigendem Buchenanteil zu erkennen. Dies ist sowohl bei der Buche im B_D Mischbestand als auch bei der Fichte im B_F Mischbestand der Fall. Die Douglasie zeigt wenig Effekt und bleibt über den gesamten Mischungsgradienten auf gleicher Höhe, während die Fichte den selben Trend wie die Buche zeigt. Das Kronenvolumen der Fichte ist im Reinbestand sowie mit $25 \%$ Buchenanteil ähnlich und sinkt dann mit einem erhöhten Buchenanteil. Dahingegen ist dieser Effekt im symmetrischen Kronenmodel Abbildung 38 nicht vorhanden. Hierbei haben in allen Fällen die Baumarten zwar einen Vorteil von der Mischung in Form von erhöhtem Volumen, allerdings verändert sich dieser nicht in Abhängigkeit der Mischungsanteile der Buchen. 


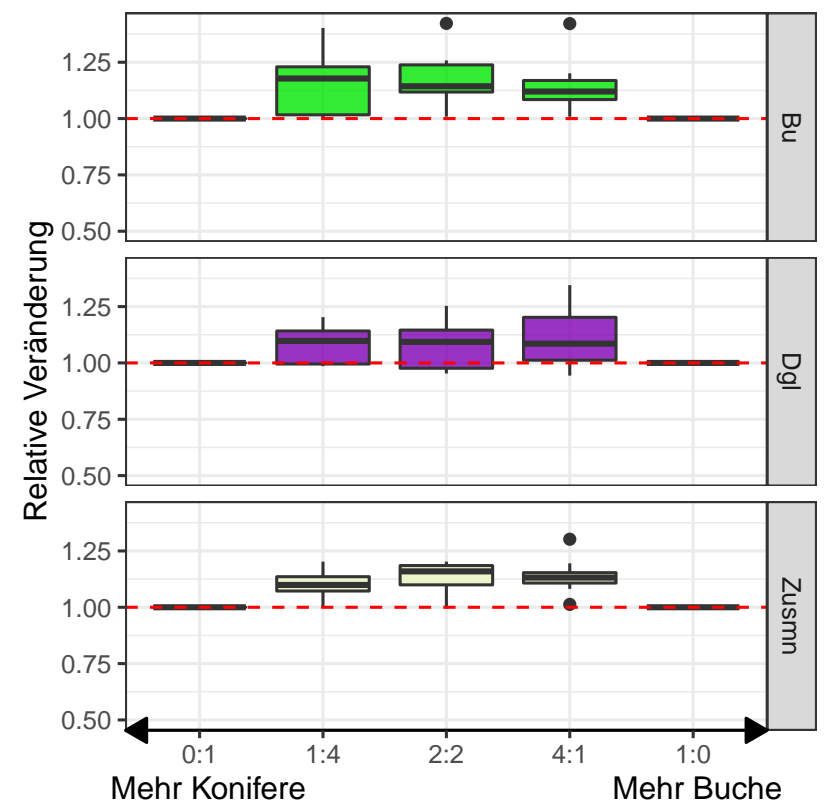

Art
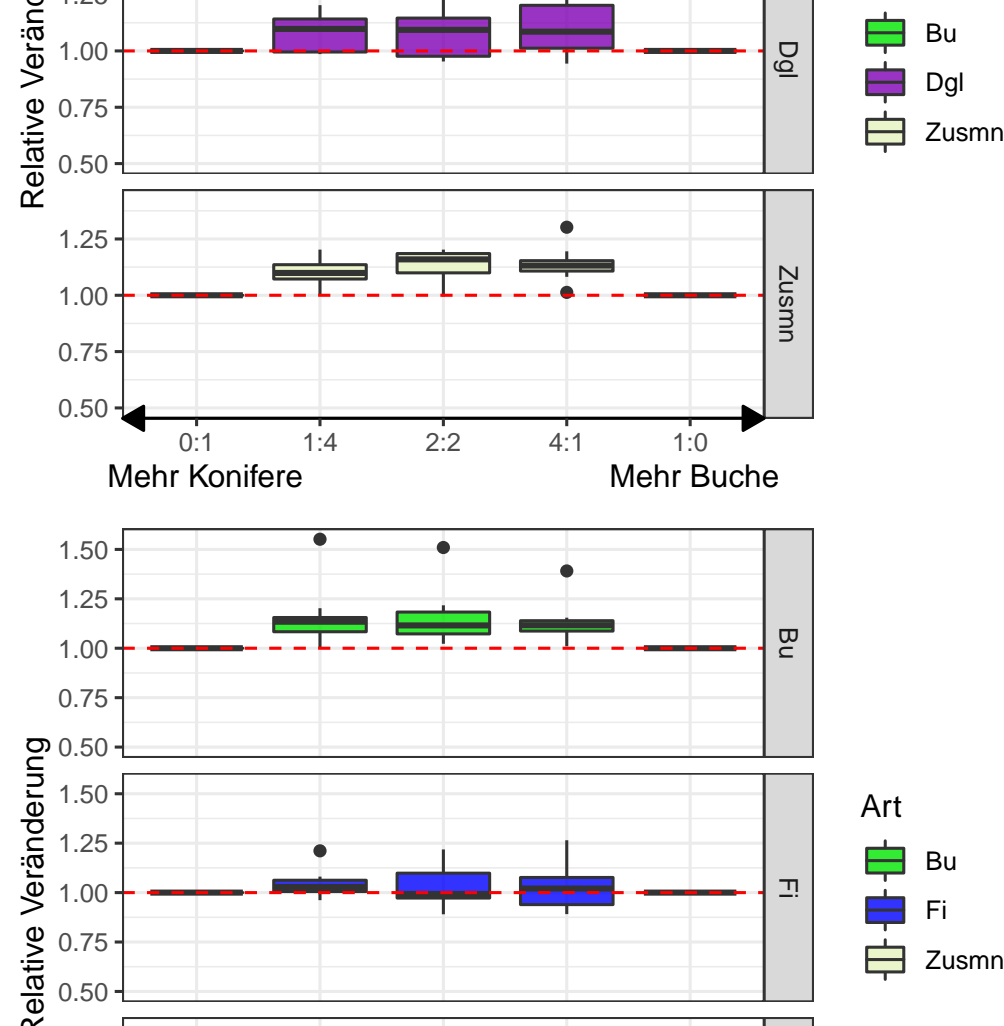

Art

官 $\mathrm{Bu}$

$\mathrm{Fi}$

官 Zusmn

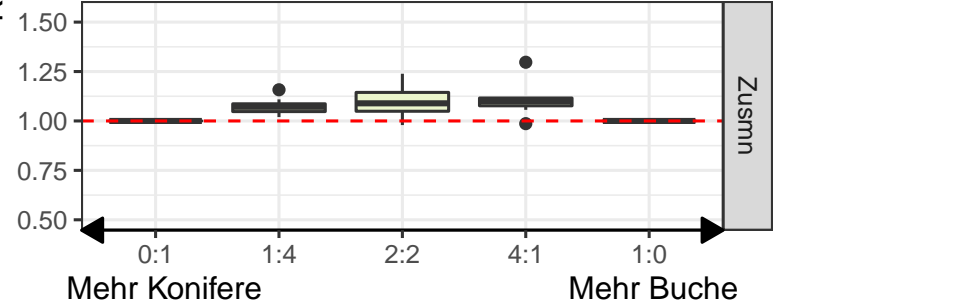

Abbildung 38: Kronenvolumen mit symmetrischer Kronenform. Relative Veränderung bei generierten Mischbeständen mit Buche/Konifere im Vergleich zu Reinbeständen, welche auf der roten Linie mit 1,0 angesiedelt sind. Die Baumarten haben in den generierten Mischbeständen eine variierende G/ha (25\%, 50\%, 75\%), jedoch immer die selbe Bonität und Alter wie die zugehörigen Reinbestände. 


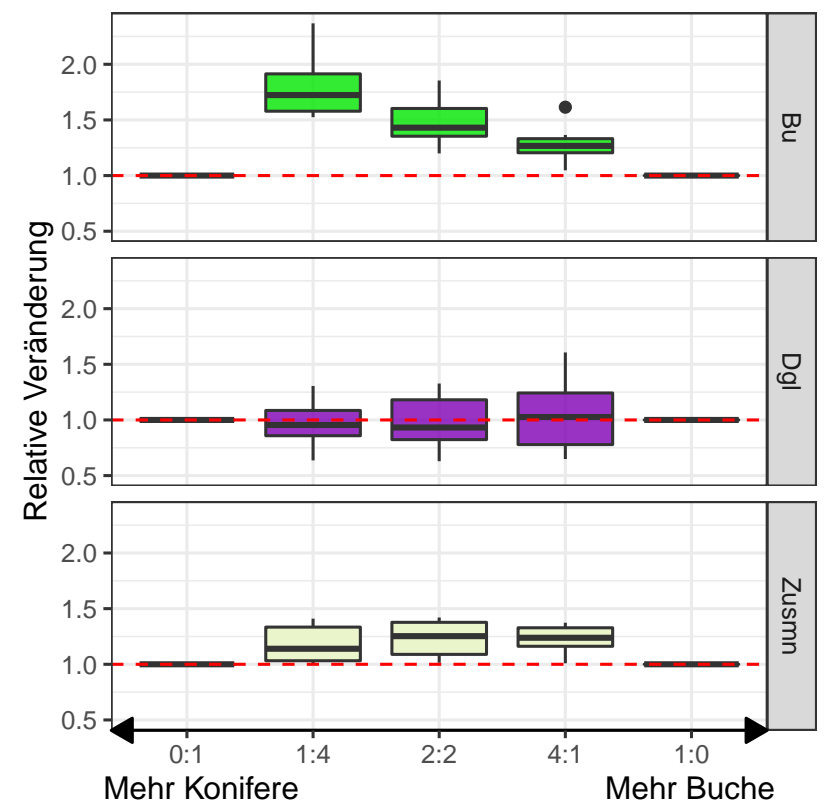

Art

\section{官 $\mathrm{Bu}$}

Dgl

官 Zusmn
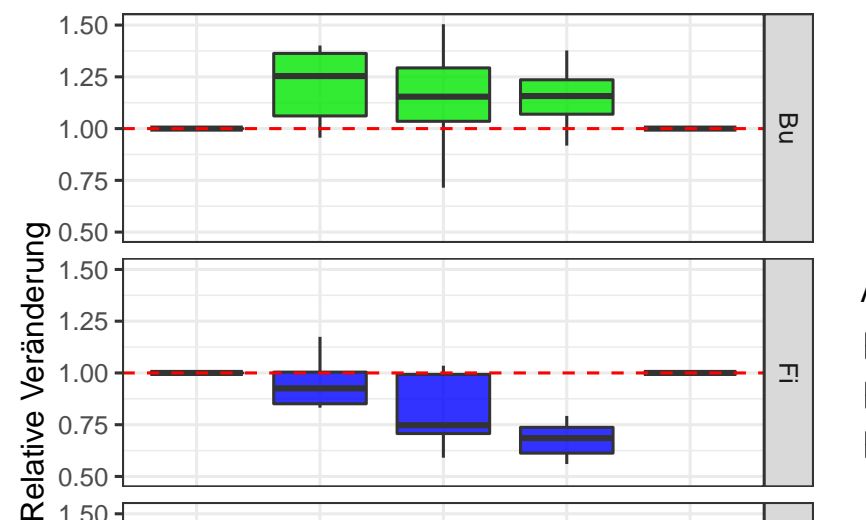

Art

官 $\mathrm{Bu}$

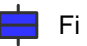

官 Zusmn

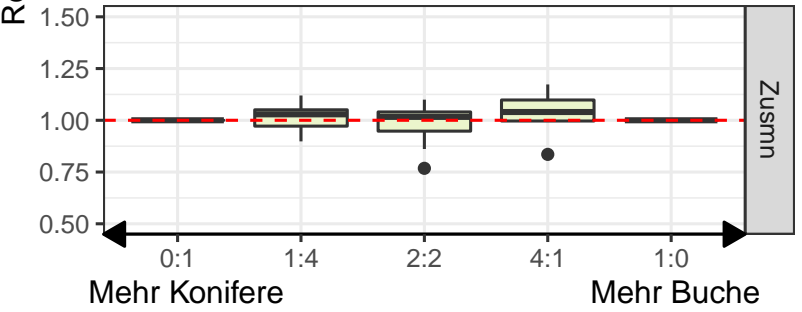

Abbildung 39: Kronenvolumen mit asymmetrische Kronenform aus Kronenmodell. Relative Veränderung bei generierten Mischbeständen mit Buche/Konifere im Vergleich zu Reinbeständen, welche auf der roten Linie mit 1,0 angesiedelt sind. Die Baumarten haben in den generierten Mischbeständen eine variierende G/ha $(25 \%, 50 \%, 75 \%)$, jedoch immer die selbe Bonität und Alter wie die zugehörigen Reinbestände. 
Trends in den Daten: bei der Betrachtung der Verläufe der relativen Veränderungen zeigt sich in mehreren Fällen ein Trend. Die Trends sich deutlicher beim asymmetrischen Kronenmodell zu erkennen. Im symmetrischen fallen diese nur gering aus und sind meistens eher horizontal verlaufend. Zunächst wird der Trend bei der Buche betrachtet. Von links nach rechts (geringer Buchenanteil $\rightarrow$ hoher Buchenanteil) ist in den meisten Fällen (in Abbildung 37 und bei B_F in Abbildung 36) ein Abwärtstrend erkennbar. Das heißt, dass die Buche die größten beleuchteten Kronenoberflächen hat, wenn $75 \%$ des Bestandes mit Koniferen bedeckt ist (nach G/ha). Ein ähnlicher Trend ist bei den Koniferen zu sehen: Bis auf die Douglasie in Abbildung 36 ist auch hier ein abfallender Trend in der beleuchteten Kronenmantelfläche zu erkennen. Somit bedeutet ein erhöhter Mischungsanteil von Buche für beide Baumarten eine geringere relative beleuchtete Kronenmantelfläche. Interessant ist allerdings, dass bei der Addition der beiden Baumarten (Zusmn) ein umgekehrter Trend in den Ergebnissen zu erkennen ist. Während also der Anteil der Buche von links nach rechts steigt, verringert sich zwar die relative beleuchtete Kronenmantelfläche bei beiden Baumarten, die gesamte beleuchtete Kronenmantelfläche steigt jedoch, da der erhöhte Anteil an Buchen im Bestand einen größeren Effekt auf die Gesamtoberfläche hat als die Verringerung innerhalb der Buchen.

Bei den Kronenvolumen zeichnen sich die gleichen Trends ausschließlich mit dem asymmetrischen Kronenmodell ab. Der Trend geht hierbei in ein abfallendes Kronenvolumen bei steigendem Buchenanteil. Das heißt, je mehr Buche im Bestand steht, desto kleiner werden die Kronen von Buche bei B_D und Fichte bei B_F. Die Fichte ist hierbei besonders stark betroffen und hat bei einem Buchenanteil von $75 \%$ nur noch $70 \%$ des Kronenvolumens verglichen zum Fichten-Reinbestand. Trotzdem zeigt das Gesamtvolumen der Bestände einen leicht erhöhten Verlauf in allen Kombinationen. Ähnlich wie bei der beleuchteten Kronenmantelfläche sorgt hier der höhere Buchenanteil für einen Ausgleich der kleineren relativen Kronenvolumen. Die Kronen sind im Vergleich zu 25\% Buchenanteil relativ gesehen bei 
75\% Buchenanteil zwar kleiner - aber das immer noch erhöhte realtive Kronenvolumen von 1,15 gleicht das geringere Volumen bei den Fichten 0,7 aus, da diese nur $25 \%$ des Bestandes ausmachen. Im symmetrischen Modell haben wir dagegen ein gleichbleibend hohes Kronenvolumen ohne einen erkennbaren Trend. 


\subsection{Vergleich geschätzter Kronenradien mit Kronenablotungen auf NW-FVA Versuchsflächen}
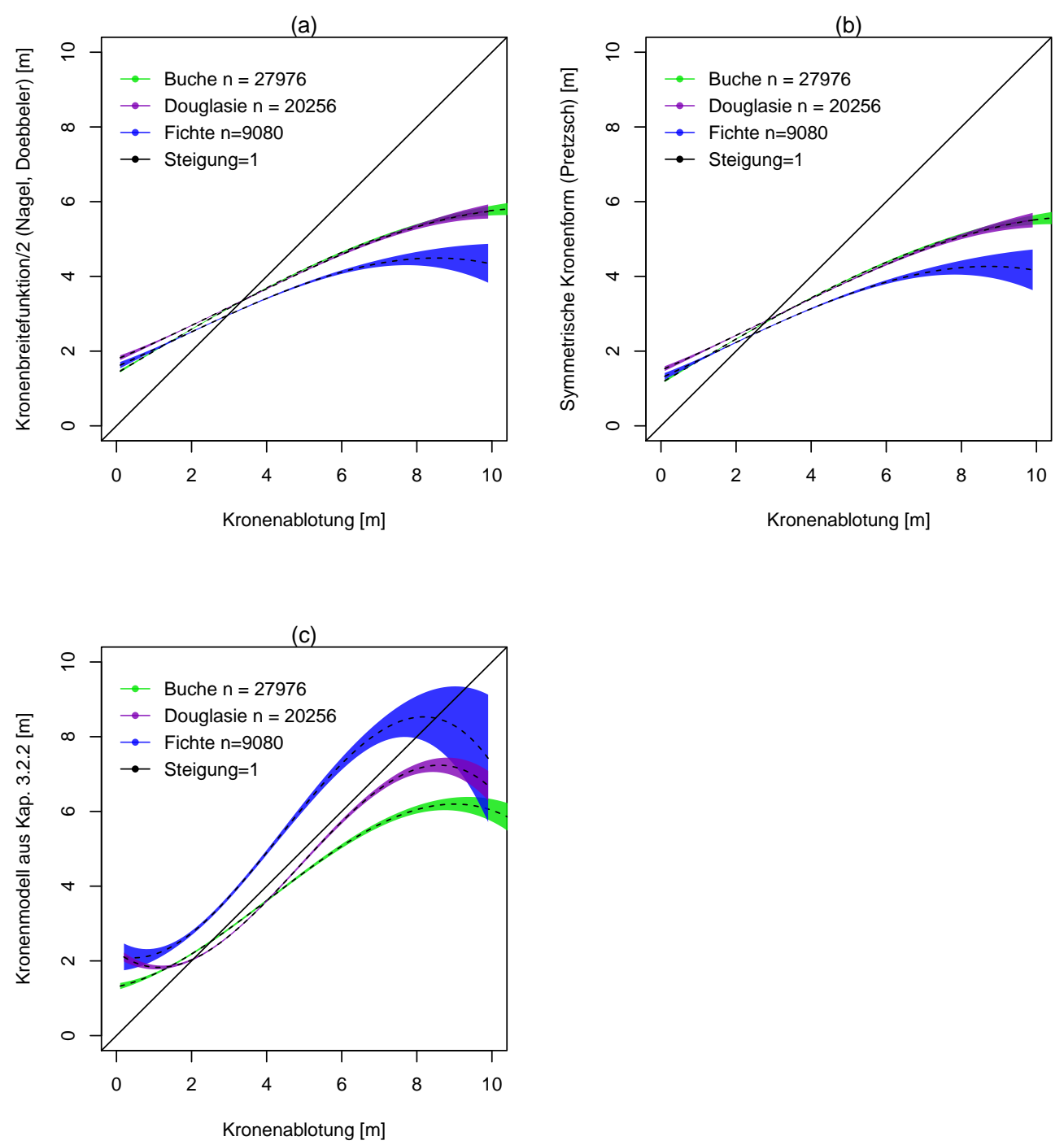

Abbildung 40: Zusammenhang der modellierten Kronenradien aus zwei Kronenfunktionen ( a (Nagel, 1999; Döbbeler et al., 2006), b (Pretzsch, 1992a)) sowie (c) dem Kronenmodell aus Kap. 3.2.2 mit Kronenablotungen aus langfristig aufgezeichneten Versuchsflächendaten der NW-FVA. Die nicht lineare Regressionsfunktion ist ein Polynom 3. Grades mit eingezeichnetem 95\% Konfidenzintervall. 
Der beschriebene Zusammenhang in Abbildung 40 zeigt die abgeloteten Kronenradien auf der X-Achse und die zugehörigen geschätzten Kronenradien mit drei unterschiedlichen Methoden auf der Y-Achse. Verglichen werden hierbei die Einzelmessungen der Kronenradien, nicht der mittlere Kronenradius. Die Vergleichsmethoden für die Schätzung der Kronenradien sind: Kronenbreitefunktionen für die Baumarten Buche, Fichte, Douglasie (Nagel, 1999; Döbbeler et al., 2006), die symmetrischen Kronenformen aus Pretzsch (1992a), sowie das in diesem Projekt erstellte Kronenmodell aus 3.2.2. Die gerade schwarze Linie beschreibt den optimalen Zusammenhang der beiden Messgrößen mit einer Steigung von 1. Die Kronenbreitefunktion sowie die symmetrische Kronenform haben einen fast identischen Verlauf. Bis zu einem Kronenradius von $3 \mathrm{~m}$ wird dieser von den beiden Methoden überschätzt und bei höheren Kronenradien unterschätzt. Die Funktionen haben einen Grenzwert, sodass höhere Radien als 5 - 6 m bei der Buche und Douglasie und 4-5 m bei der Fichte die Maximalwerte darstellen. Bei realen Messungen aus Kronenablotungen kommen hingegen Kronenradien von bis zu 10 m vor, je nach Beschaffenheit und Ovalität des Baumes. Die Regressionsfunktion des Kronenmodells aus 3.2.2 verläuft bei Fichte und Douglasie etwas dichter an der schwarzen Optimallinie als bei der Buche. Die Steigung ist im Mittelteil der Kurven annähernd 1, während es bei den Maximalwerten zu einer größeren Streuung kommt, was vor allem bei dem größeren 95\% Konfidenzintervall der Fichte zu erkennen ist. Die Regressionsfunktion der Buche zeigt in Abbildung (c) einen anfangs besser passenden Verlauf und bei höheren Kronenradien einen ähnlichen Verlauf wie in der Kronenbreitefunktion in Abbildung (a) und der symmetrischen Kronenform in Abbildung (b). Die maximalen geschätzten Kronenradien erreichen bei allen Varienten mit Buche Werte von 5 - 6 m (a, b) und 6 m (c). Das Ergebniss zeigt, dass mit dem Kronenmodell bei Buche, Fichte und Douglasie realistischere Kronenradien geschätzt werden, vor allem wenn diese über den Maxmialwerten der Kronenfunktionen liegen. Dieser Vergleich sagt nichts über die Güte der Kronenbreitefunktionen (a) und (b) aus, da diese die Kronenbreite, bzw. den mittleren 
Kronenradius (Kronenbreite / 2, hier verwendet) errechnen. Diese sind nicht für die Schätzung von Asymmetrien geeignet, sondern liefern gemittelte Kronenmaße. Aus diesem Grund können mit den Funktionen auch keine Extremwerte geschätzt werden, während mit dem Kronenmodell aus Kap. 3.2.2 diese besonders hohen Kronenradien wesentlich besser geschätzt werden können.

\section{Diskussion}

\subsection{Beurteilung der Versuchsflächen und des Datenmaterials auf den Versuchsflächen}

Die Versuchsflächen wurden größtenteils im Jahr 2017 vor Beginn der Projektlaufzeit aller Teilprojekte ausgewählt. Geeignete Flächen zu finden stellte aufgrund der Anforderungen an das Vorhandensein aller fünf Bestandestypen eine Herausforderung dar. Hierbei wurde auf eine räumlich dichte Lage der Bestände innerhalb der Quintetts geachtet. Wegen dieser einschränkenden Bedingungen in der Versuchsflächenauswahl schwanken die mittleren Alter der Bestände in einem Bereich von 55 - 96 Jahren (Tabelle 3 in Abschnitt 2.5.3). Diese Unterschiede sind einerseits gut, um mit dem hier vorhandenen Modellansatz ein größeres Spektrum an Kronen schätzen zu können. Denn hierbei ist besonders die Größe und somit auch das Alter eines Baumes ausschlaggebend. Andererseits hat dieser Unterschied den Nachteil, dass der Vergleich der Quintetts untereinander schwerer möglich ist. Ein Vergleich muss die Altersunterschiede berücksichtigen. Außerdem wurden die Bestände alle durchforstet und haben somit in der Vergangenheit eine unterschiedliche Behandlung erfahren, was es besonders wichtig macht, möglichst viele Versuchsflächen zu haben, damit einzelne forstliche Eingriffe in der Vergangenheit keine zu großen Auswirkungen auf die Gesamtdaten haben. Wenn zum Beispiel ein Bestand mit Douglasien im Alter 50 nur einmal vorkommt und dort durch den ansässigen Förster besonders stark freigestellt wurde, hätte dies einen großen Effekt auf das Kronenmodell bei der Schätzung von 50 jährigen Douglasienkronen. 
Die Versuchsflächendaten wurden in diesem Projekt mit Datenmaterial aus der NW-FVA ergänzt (Abbildung 19 bis Abschnitt 3.1.4) und die aus den Modellen resultierende Kronenbreite mit langfristigen Kronenablotungen verglichen (Abschnitt 3.4). Somit konnten die zuvor beschriebenen Nachteile verringert oder aus-

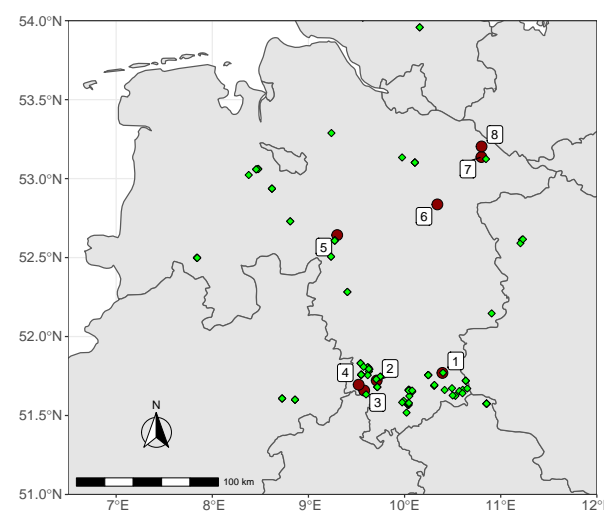
geglichen werden. Das Datenmaterial der NW-FVA ist sehr umfangreich und auf vielen der Flächen existieren wiederholte Datenerhebungen. Es umfasst zahlreiche Bestände aus den gleichen Wuchsgebieten (siehe Abbildung 41: Verteilung der Versuchsflächen der NW-FVA (grün) und des GRK 2300 (rot) Abbildung 41), wie sie auch für die Quintetts verwendet wurden (Solling, Harz, Norddeutsche Tiefebene). Zudem fließen hier auch Wuchsreihenversuche der NW-FVA ein, welche mit Buche/Douglasie angelegt wurden und schon in anderen Forschungsarbeiten untersucht wurden (Biber, 1996; de Wall, 1995; Pretzsch, 1992b). Viele Aufnahmen stammen aus dem Solling und Harz, in denen sich auch die Hälfte der GRK2300 Versuchsflächen befinden. Im Flachland sind die Flächen der NW-FVA sowie des GRK2300 weiträumiger verstreut. Da jedoch im nördlichen Teil Niedersachsens durch die eiszeitlichen Landschaftsformungen nur geringe Höhenunterschiede und häufig Podsole und Histosole vorhanden sind, ist die Streuung dort weniger ausschlaggebend, als der Unterschied der Norddeutschen Tiefebene zu den Mittelgebirgslagen Solling und Harz. 


\subsection{Beurteilung des entwickelten Modellansatzes}

Der Modellansatz, beschrieben in 3.2.2, wurde im Laufe der zweiten Projekthälfte entwickelt. Er beruht auf den Kronenfotos, welche zunächst für den Zweck der Kronenform- und Strukturuntersuchung erfasst wurden. Eine wichtige Entscheidung war die Wahl der Funktion, die die Kronenform beschreiben soll. Besonders interessant sind hier vor allem Häufigkeitsdichtefunktionen, welche möglichst wenige Parameter benötigen, um ein Kronenprofil annähernd darstellen zu können. Es gibt mehrere Funktionen, welche sich mehr oder weniger gut für die Kronenformmodellierung eignen. In Mirzaei et al. (2016) wurden die Verteilungsfunktionen „Beta“, „Exponential“, „Gamma“, „Lognormal“, „Normal“ und „Weibull“ für Kronenformen der Eiche miteinander verglichen und die zwei-parametrische Weibullfunktion für die Kronenformmodellierung als am geeignetsten befunden. Als etwas weniger signifikant, aber immer noch ausreichend, stellte sich die Gammaverteilung heraus. In Ferrarese et al. (2015) wurden die Kronenprofile mit terrestrischen Laserscans erfasst und daraufhin die Punktdaten des Kronenrandes verwendet, um an diese eine Weibull-Verteilung sowie Beta-Verteilung zu fitten. Die verwendeten Punktdaten hatten nach ihrer Abstraktion aus den TLS Daten einen vergleichbaren Informationsgehalt wie die hier erfassten Kronenprofile aus den Fotos (siehe Abschnitt 3.2.1). Auch wurden Kronenprofile von Koniferen (verschiede Kiefernarten) mit Hilfe der Weibull-Verteilung schon von Schreuder und Swank (1974) modelliert. Dort diente die Blattmasse des Kronenquerschnitts zur Prädiktion des vertikalen Kronenprofils. Ein ähnliches Beispiel für die Verwendung der WeibullVerteilung liefert Mori und Hagihara (1991). Sie haben die vertikalen Blattflächen in Abschnitte unterteilt und deren Verteilung mit einer Weibull-Dichtefunktion beschrieben. In Mehtätalo (2006) diente die Weibull-Verteilung zur Schätzung der Kronenradien in Waldbeständen, um Kronenüberlappungen mit TLS oder Luftbildern zu Untersuchen. Aber auch andere Verteilungsfunktionen haben mitunter zur Beschreibung des Kronenprofils gedient. In einer Forschungsarbeit von Williams et al. (2017) wurde für die Modellierung der Krone von Birke und Kiefer eine 
Beta-Verteilung verwendet. In Mirzaei et al. (2016) wurde die Gamma-Verteilung für eine Kronenprofilmodellierung getestet und bestätigt.

Aufgrund der Literaturinformationen über die Verwendung einer Häufigkeitsdichtefunktion fiel die Entscheidung auf die zwei-parametrische Weibull-Dichtefunktion, da diese in der Mehrzahl für eine Kronenprofilmodellierung verwendet wurde. Auch das Spektrum der Baumarten, deren Krone damit modelliert wurde, war hier am höchsten. Dies ist wichtig, da mit dieser Funktion die drei Baumarten Buche, Douglasie und Fichte modelliert werden, welche grundsätzlich schon sehr unterschiedliche Kronenformen haben (siehe direkten Vergleich der Kronenprofile in Abschnitt 3.2.1 - Abbildung 27).

Das Modell ist ein generalisiertes additives Modell und wurde in $\mathrm{R}$ mit dem Paket „mgcv“ (Wood, 2001) entwickelt. Mit einem generalisierten additiven Modell (GAM) lassen sich komplexe Beziehungen hoch dimensionaler Daten mit nicht linearen Anteilen modellieren (Adler, 2010). Zweck des Modells ist es, die benötigten Parameter für die Weibull-Dichtefunktion (zur Darstellung des Kronenprofils) anhand der Prädiktorvariablen zu schätzen. Aufgrund der Vielzahl von beeinflussenden Effekten, welche nicht zwingend linearer Natur sind, fiel die Entscheidung auf die Verwendung von GAMs. Gerade die Strukturuntersuchungen in Mischbeständen können nichtlineare Effekte beinhalten, welche erst mit einem solchen Modell dargestellt werden können. Da mittels mgcv: :gam (Wood, 2011) und der Option select=TRUE gearbeitet wurde, sind die selektierten Modellkomponenten in 3.2.2 aufgeführt. Die verwendete Option macht es „mgcv“ möglich, die Prädiktoren selbst zu wählen und ihren Einfluss auf die Modellfunktion zu variieren (Wood und Augustin, 2002). Im Vorfeld wurde mit manueller auf „Akaike information criterion“ (AIC) (Sakamoto et al., 1986; Hu, 2007) basierter Auswahl der signifikanten Prädiktoren gearbeitet, was sich jedoch aufgrund der unterschiedlichen Baumartenkombinationen als nicht optimal herausgestellt hat. Bei der manuellen Selektion wird mit allen Modellkomponenten gestartet und geschaut, ob sich der AIC für ein Modell bei Entfernung einer Modellkomponente verschlechtert. Tut er 
dies nicht, dann hat die Modellkomponente keinen Einfluss auf die Güte des Modells und wird nicht mit aufgenommen. Die in 3.2.2, Tabelle 16 vorgestellte Tabelle zeigt die selektierten Modellkomponenten und deren geschätzten Freiheitsgrade. Die Vielzahl an Modellkomponenten kommt zustande, da es für jeden Prädiktor die verschiedenen Baumartenkombinationen $\left(\mathrm{B} \_\mathrm{B}, \mathrm{B} \_\mathrm{F}, \ldots, \mathrm{D} \_\mathrm{B}\right)$ gibt. Diese Kombinationen sind ausschlaggebend für das Modell, um das Kronenprofil auf die Nachbarschaft eines Baumes reagieren zu lassen.

Bei Verwendung des Modells gibt es Einschränkungen, welche auf die zugrundeliegenden Datensätze zurückzuführen sind. Der Gültigkeitsbereich des Modells ist auf die Altersspanne, Baumgröße und Kombination der GRK2300 Versuchsflächen beschränkt, denn aus Daten dieser Flächen wurde das Modell gefittet. In diesem Projekt waren die Bestände nicht zweischichtig, sodass der Fokus hauptsächlich auf dem oberen Kronenraum lag. Deshalb sind unterständige Bäume größtenteils nicht in das Modell eingeflossen. Es ergeben sich daher für besonders kleine Bäume Kronenprofile, welche in bestimmten Wuchskonstellationen Extremwerte annehmen können. Zur Prävention dieser Effekte werden die modellierten Kronenprofile nur bei Bäumen verwendet, welche sich mit ihrem BHD innerhalb der BHD-Spanne des Gesamtdatensatzes befinden. Diese Einschränkung findet in Abschnitt 3.2 Verwendung, wenn es darum geht, die beleuchtete Kronenoberfläche oder das Kronenvolumen zu berechnen. Zudem wurde die Schätzung der Shapeund Scale-Parameter auf den minimalen Wert von Shape $=1$,4; Scale $=7$ beschränkt. Dies soll extreme Kronenbreiten verhindern, welche bei seltenen, nicht im Modell enthaltenen Nachbarschaftsverhältnissen vorkommen können. War der Shape Parameter unter 1,4 kam es vor, dass das Modell sehr hohe Kronenbreiten für die Wuchskonstellation geschätzt hat. Die Einschränkungen des Gültigkeitsbereiches würden auch bei Verwendung des Modells in Waldwachstumssilmulatoren, wie beispielsweise TreeGrOSS, im derzeitigen Stand des Kronenmodells benötigt werden. Für eine Verbesserung des Gültigkeitsbereiches wäre es sinnvoll, weitere Bäume in anderen Alters und Größenklassen zu erfassen und somit Kronenpro- 
file in weiteren Bestandessitutionen modellieren zu können. Dadurch könnte das Kronenmodell auf beliebige andere Versuchsflächen mit den gefitteten Baumarten übertragen werden.

\subsection{Geschätzte maximale Kronenradien}

Die Schätzung der maximalen Kronenradien mittels Kronenmodell und deren Vergleich mit Kronenablotungen dient der Einordnung der Modellergebnisse. Die in Abschnitt 3.4 vorgestellten Ergebnisse zeigen diesen Zusammenhang im Vergleich zu zwei anderen Schätzmethoden für die mittleren Kronenbreiten (verglichen werden die Kronenbreiten / 2 mit den Kronenradien des Kronenmodells). Die bessere Übereinstimmung der Regressionsfunktionen des Kronenmodells bei Kronenradien von Buchen, Fichten und Douglasien ist eine Folge der Anpassungsfähigkeit des Kronenmodells. Wenn als erklärende Variablen der BHD (Hasenauer, 1997; Nagel und Schmidt, 2006) und zusätzlich die Baumhöhe (Pretzsch et al., 2002) verwendet wird, liefert ein entsprechendes Model bzw. die darauf beruhende Kronenbreitefunktion eine mittlere Kronenbreite. Das Kronenmodell hingegen kann diese in Abhängigkeit der Standsituation eines Einzelbaumes schätzen, in welcher auch die Nachbarbäume und deren Eigenschaften mit berücksichtigt werden. Da bei einer symmetrischen Kronenform ausschließlich mittlere Kronenradien/Kronenbreiten Verwendung finden, ist zu erwarten, dass die dafür verwendeten Funktionen keine Extremwerte darstellen können. Folglich gibt es bei besonders kleinen realen Kronenradien eine Überschätzung des Kronenradius und bei großen realen Kronenradien eine Unterschätzung. Im Gegensatz dazu kann das Kronenmodell aus 3.2.2 diese Extremfälle berücksichtigen, was zu einer besseren Übereinstimmung mit den abgeloteten Kronenradien führt. Dieser Effekt ist bei der Schätzung von Kronenradien der Buche etwas abgeschwächt. Das Kronenmodell führt hierbei etwas mehr zu einer Unterschätzung bei großen Kronenradien als es bei Fichte und Douglasie der Fall ist. Bei dieser Auswertung ist zu bedenken, dass einzelne Kronenradien miteinander korreliert werden. Eine Aussage über die 
gesamte Kronengröße eines Baumes wird hierbei nicht getroffen. Die Kronengröße ergibt sich erst aus der Gesamtheit des Kronenkörpers, welcher unter anderem auch die maximalen Kronenradien beinhaltet (Ergebnisse dazu in Abschnitt 3.3).

Bei Verwendung von fotogrammetrischen Vermessungen ist $\mathrm{zu}$ bedenken, dass der maximale Kronenradius anders ermittelt wird, als bei einer Kronenablotung. Bei einem Foto der vertikalen Krone wird hierbei der längste Ast vermessen (siehe Beispiel 2 auf Seite 18). Durch eine heterogen strukturierte Krone kann es sein, dass dies nicht exakt die selbe Richtung ist, in welche auch abgelotet wurde. Wenn also die Ablotung in Richtung Nord $0^{\circ}$

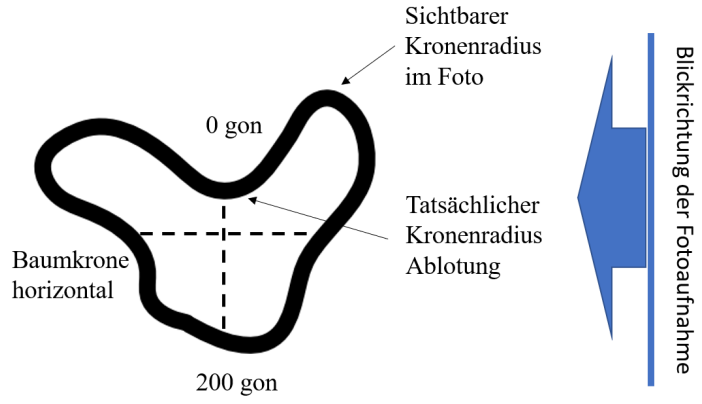

Abbildung 42: Beispiel für die unterschiedliche Erfassung des Kronenradius bei Kronenablotung und durch Auswertung aus Fotos und den daraus resultierenden Fehler.

stattfand, aber der längste Ast in dieser Richtung leicht geneigt in $20^{\circ}$ Richtung Nord-Nord-Ost zeigt, dann wird eine Ablotung diesen nicht erfassen. Auf dem Kronenprofilbild würde dieser jedoch als längste maximaler Kronenradius erfasst werden. Denn von einem seitlichen Betrachtungswinkel aus erscheinen auch die davorliegenden oder dahinterliegenden Äste (um die $0^{\circ}$ Ausrichtung in diesem Beispiel) als maximale Kronenausdehnungen (sieheAbbildung 42). Aus diesem Grund wurde nicht erwartet, dass das Kronenmdell überhaupt für die vergleichsweise gute Vorhersage von maxmialen Kronenradien geeignet ist, bzw. mit Kronenablotungen vergleichbar ist. Es könnte sein, dass solche Ausformungen der Krone nur selten vorkommen, sodass der Vorteil des nachbarschaftssensitiven Kronenmodells gegenüber den mittleren Kronenradien der Kronenbreitefunktionen noch wesentlich größer ist, als die Fehler, welcher durch den Blickwinkel auf den Kronenrand enstehen kann. 
Gegebenenfalls könnte eine Vergrößerung des Eingangsdatensatzes für das Kronenmodell auch die Vorhersage der maximalen Kronenradien für die Buche weiter verbessern. Der Datensatz der NW-FVA, welcher hier für den Vergleich der Kronenablotungen mit den Schätzungen verwendet wurde, beinhaltet viele verschiedene Bestandesalter und Baumgrößen. In das Modell sind hingegen nur Daten aus den Beständen des GRK2300 (Versuchsflächenübersicht Abschnitt 2.5.3) eingeflossen, was die Übereinstimmung der Kronenradien im Extrapolationsbereich des Modells wahrscheinlich erschwert.

\subsection{Einfluss der direkten Nachbarschaft auf die funktionalen Merk- male von Buche, Douglasie und Fichte}

Ergebnisse für den Beleg der Hypothese H1 wurden im Teil Abschnitt 3.1 dargestellt. Die Kombination der Versuchsdaten aus den GRK2300 Flächen mit dem Datensatz aus der NW-FVA hat die Ergebnisse verbessert, da so ein wesentlich größerer Datensatz verfügbar war. Bei der Betrachtung der Versuchsflächenverteilung in Abbildung 41 ist zu erkennen, dass die Daten der NW-FVA aus vergleichbaren Standorten wie die des GRK2300 erhoben wurden (siehe auch Abschnitt 4.1). Die Kernaussage aus Abbildung 19 in Abschnitt 3.1 ist, dass die Kronenbreite der Buchenkronen, bei gleichem BHD und Höhe, in Mischung mit beiden Koniferenarten größer ist, als im Buchen-Reinbestand. Zugleich gibt es bei den Koniferen keinen signifikanten Unterschied der Kronenbreiten in Rein- oder Mischbestand. Zu diesem Ergebnis kommt auch eine Forschungsarbeit von Barbeito et al. (2017), in denen mit TLS Daten die Kronengößen von Buchen in Reinbestand und Mischung mit Kiefern untersucht wurden. Da die Kronengröße von Bäumen in einem Waldbestand mit der Produktivität von einzelnen Individuen korreliert (Stenberg et al., 1994; Wyckoff und Clark, 2005; Kramer, 1986), könnte die größere Kronenbreite der Buchen, bei gleichbleibender Kronenbreite der Koniferen, zu einer vergrößerten Gesamtproduktivität des Mischbestandes führen. Dies würde voraussetzen, dass in einem Waldbestand der Kronenraum ggf. nicht optimal genutzt wird, wenn die 
Buche in einem Reinbestand steht. Hingegen könnte die Buche in Mischung unter Koniferen noch genug Licht finden, um auch diesen Standort noch zu nutzen und dies somit zu einer erhöhten Raumnutzung im Bestand führen.

Die Untersuchung der Kronenbreite wurde in Abschnitt 3.1 durch die horizontale Kronenausdehnung (Abschnitt 3.1.3) sowie die Kronenasymmetrie (Abschnitt 3.1.4) ergänzt. Diese soll einen Rückschluss auf die Kronenplasitizität geben sowie Unterschiede dieser Anpassung in Rein- und Mischbestand erfassen. In Schröter et al. (2012) wurde festgestellt, dass die Kronenplastizität der Buche vergleichsweise hoch ist und sie diese auch noch in hohen Altern beibehält. Dies führt nach Schröter et al. (2012) zu einer optimalen Ressourcennutzung für die Buche und geringere intraspezifische Konkurrenz, da die Buche Lücken zuerst effektiv nutzt bevor die Kronen ineinander wachsen und sich durch mechanische Interaktion/Abrieb abgrenzen. Die relativen gemittelten Kronenradien (Abbildung 21) zeigen diese Plastizität nicht direkt, aber es lässt sich eine Asymmetrie vor allem im Mischbestand feststellen. Zunächst ist hier die Kronenausdehnung zu sehen, welche im Mischbestand bei Buchen größer ist als im Reinbestand. Die Kronenausdehnung ist nicht symmetrisch um den Baum, sondern erstreckt sich weiter in südlicher Richtung. Dies wird von Untersuchungen durch Vorreyer (1997); Biber (1996); de Wall (1995) bestätigt, welche ähnliche Effekte in Buchen-Douglasien Mischbestandswuchsreihen gefunden haben. Es wird angenommen, dass vor allem die Buche in den Mischbeständen die Möglichkeit hat, sich optimal zur Sonne hin zu positionieren. Die Fähigkeit zu dieser Anpassung spricht somit indirekt für eine plastischere Krone der Buche.

Die Höhe der maximalen Kronenbreite in Abschnitt 3.1.2 soll die vertikale Bestandesstruktur beschreiben. Die hierbei gefundene Variablilität der Buche ist eine Bestätigung der Plastizität der Buchenkrone (Schröter et al., 2012) auch in vertikaler Form. Hier zeigt sich bei dem Vergleich der relativen Höhen der maximalen Kronenbreiten, dass die Buche im Stande ist, ihren Kronenschwerpunkt bei Mischung mit Fichte und besonders Douglasie nach unten zu verschieben. Mög- 
licherweise geschieht dies in Mischung deshalb, da die Buche den Kronenraum unter der Douglasie und Fichte noch nutzen kann, während sie neben anderen Buchen ihre maximale Kronenbreite möglichst weit nach oben verschiebt, um mit den umstehenden Buchen mithalten zu können (hohe intraspezifische Konkurrenz, niedrigere interspezifische Konkurrenz). Gleichzeitig hat die Buchenkrone, wenn sie im Reinbestand vertikal höher gelegen ist, eine insgesamt kleinere Kronenbreite, wie in Abschnitt 3.1.3 gezeigt wurde. Die Buchen verschieben im Reinbestand ihre Krone nach oben, während sie speziell unter der Douglasie ihre Krone unter der Douglasienkrone lassen und die dadurch erhöhte Beschattung mit einer größeren horizontalen Kronenbreite kompensieren.

Die Untersuchung der Ovalität in Abschnitt 3.1.4 ergab eine höhere Ovalität der Buche im Mischbestand (Abbildung 22) und im Gegensatz dazu eine geringere Ovalität (also eine kreisrundere Krone) der Douglasie im Mischbestand (Abbildung 23). Der Effekt der geringeren Ovalität der Douglasie in Mischung mit Buche könnte mit der Wuchshöhe der Douglasie in Zusammenhang stehen. Es könnte sein, dass die Douglasie im Mischbestand mit Buchen eher die Möglichkeit hat eine kreisrunde Krone auszubilden, da sie durch ihr schnelleres Wachstum mit ihrer Krone über den Buchen hinausragt (Thomas et al., 2015). Zu dieser Annahme passen auch die in Abschnitt 3.1.2 gefundenen Unterschiede in der Höhe der maximalen Kronenbreite, in der die Douglasienkrone mit ihrer maximalen Kronenbreite über der Buchenkrone ist und die Buche speziell in Mischung mit Douglasie eine tiefer liegende maximale Kronenbreite hat. In direkter Nachbarschaft zu anderen Douglasien hingegen hat die Douglasie gleichgroße Konkurrenten im oberen Kronenraum und somit häufiger eine durch Konkurrenz bedingte ovale Kronenform. Bei der Buche könnte dagegen die höhere Ovalität in Mischung mit Douglasie und Fichte damit zusammenhängen, dass die Buche hier die Möglichtkeit hat, Lücken und von den Koniferen überschatteten Kronenraum zu nutzen. Die Buche könnte bei den Koniferen also durch andere Lichtverhältnisse und die Plastizität ihrer Krone (Schröter et al., 2012) zu der höheren Ovalität neigen. Im Reinbestand hin- 
gegen kann die Buche den Kronenraum der Nachbarbuchen nicht so gut nutzen, da alle Nachbarbäume Buchen sind, welche die selben Merkmale (z.B. sehr hohe Schattentoleranz, Wuchshöhe, Kronenlänge) aufweisen. Der Kronenraum im Reinbestand ist schon besetzt und die Buche hat wenig Möglichkeiten ihre Krone zu verformen, um weiteren Raum zu nutzen.

\subsection{Mischbestandseffekte}

Die Beantwortung der Hypothese H2 - "Mischungen aus Rotbuche mit Fichte oder Douglasie führen zu einer unterschiedlichen Kronenstruktur, Wuchsraumeffizienz und Produktivität im Vergleich zum Reinbestand“ bedurfte einer genauen Untersuchung der Baumkronen. Diese wurde mit Hilfe eines Kronenmodells durchgeführt. Der Modellansatz wird in Abschnitt 4.2 beschrieben. Das Modell erlaubt eine einzelbaumbasierte und nachbarschaftsabhängige Auswertung der Kronen. Wird das Modell auf alle Baumkronen eines Bestandes angewandt, können Informationen über den Wuchsraum gewonnen werden. Da die Kronenformen in Abhängigkeit der umgebenden Bäume modelliert werden, ist der Vergleich von Rein- und Mischbeständen besser möglich, als es nachbarschaftsunabhängige Kronen zulassen würden.

\subsubsection{Reaktionsmuster der Baumkronen}

In 3.2.3 werden die Reaktionsmuster der Baumkronen exemplarisch vorgestellt. Da die Ergebnisse des Modells kontinuierlich auf die eingehenden Prädiktoren reagieren, wurden nur die Fälle dargestellt, in denen die Effekte der Nachbarschaft auf die Baumkronen auffällig waren. Die Effekte der Buchenkrone neben weiteren Buchen ist in Abbildung 30 dargestellt. Die Buchenkrone zeigt nur geringe Veränderung in Nachbarschaft zu weiteren Buchen. Im Vorfeld dieser Untersuchung wurde davon ausgegangen, dass Buchen den größten Konkurenzdruck vor allem von weiteren Buchen erfahren, da diese besonders schattentolerant sind (Peters, 1992; Felbermeier und Mosandl, 2004; Pretzsch, 1992a). Diese Erwartung wurde 
bestätigt, da die Buche in Abbildung 30 eine kleinere Krone bei dichter stehenden Nachbarbuchen hat (geringere maximale Kronenbreite). Als Vergleich dazu ist in Abbildung 31 die Buche neben einer Douglasie zu sehen. Hierbei hat die Buche dicht neben der Douglasie eine tropfenförmige Krone, während sie bei größerem Abstand wieder eine Kronenform ähnlich der Reinbestandsform bekommt.

Die Tropfenform der Buchenkrone neben der Douglasie ist ein Hinweis darauf, dass der Konkurrenzdruck dieser Bäume geringer ist und die Buche deswegen nicht versucht, ihre Krone in die oberen Bereiche auszuweiten. Sie kann hingegen durch ihre große Schattentoleranz auch neben und unter der Douglasie existieren, während es neben einer weiteren Buche eher von Vorteil ist, wenn sie ihre Krone weiter nach oben schiebt. Die Fichte hat nicht den selben Einfluss auf die Buchenkrone, wie es die Douglasie gezeigt hat. Die Kronenform der Buche ist keiner großen Veränderung in Abhängigkeit der Distanz von der Fichte ausgesetzt (Abbildung 32). Die Modellschätzungen werden durch die in Abschnitt 3.1.2 ausgewerteten Höhen der maximalen Kronenbreite bestätigt.

Auch die Kronenform der Douglasie zeigt eine Veränderung neben der Buche in Abbildung 34. Die runder verlaufende obere Kronenform bei benachbarten Buchen könnte den Effekt einer komplementären Kronenform zeigen. Ähnlich wie in Pretzsch et al., 2010, 2013 würde die Douglasienkrone durch den höheren Wuchs den Kronenraum oberhalb der Buche optimal nutzen und durch die rundere obere Kronenform mehr Nadelmasse in oberen Kronenbreichen für die Photosynthese verwendet werden. Diese komplementären Effekte, speziell bei Buche und Douglasie sind zudem eine Erklärung für die größere beleuchtete Kronenmantelfläche und das höhere Kronenvolumen in den Untersuchungen auf Bestandesebende (Abschnitt 3.3 auf Seite 74).

\subsubsection{Bestandeseffekte des Kronenmodells}

Die Simulation der Mischbestände mit den variierenden Mischungsanteilen (nach $\mathrm{G} / \mathrm{ha}$ ) wurde für die Bestandesuntersuchung verwendet, da sich so mit dem Kro- 
nenmodell am besten die Unterschiede von Rein- und Mischbestand feststellen lassen. Die simulierten Mischbestände können mit der selben Bonitäten wie die jeweiligen Reinbestände aufgebaut werden und in ihren Mischungsanteilen beliebig variiert werden. Ein derartiger Vergleich der Bestandestypen beruht nicht auf unterschiedlichen Bonitäten oder waldbaulichen Behandlungen der gegebenen Versuchsflächen, sondern nur auf den Unterschied der Kronenstruktur in den Beständen. Durch die begrenzte Versuchsflächenanzahl wäre ein direkter Vergleich der Rein- und Mischbestände nicht möglich gewesen.

Die Anwendung des Modells auf alle Individuen der Testbestände in Abschnitt 3.3 soll prüfen, ob postitive Effekte auf Bestandesebene vorhanden sind, welche sich aus den Reaktionsmustern der Einzelbäume ergeben. Positive, komplementäre Effekte in Mischbeständen können vorkommen, wenn die intraspezifische Konkurrenz geringer ist, als die interspezifische Konkurrenz (Kelty, 1992). So etwas ist beispielsweise dann anzutreffen, wenn sich die Eigenschaften der Arten unterscheiden und diese möglicherweise andere Ressourcen benötigen oder die selben Ressourcen in unterschiedlichen Zeiträumen (Lei et al., 2012; Bauhus et al., 2000, 2004; Hooper et al., 2005). Das Kronenmodell schätzt insgesamt eine größere beleuchtete Kronenmantelfläche in den Mischbeständen, welche in Abbildung 37 vor allem von der Buche verursacht wird. Die beleuchtete Kronenmantelfläche zeigt die Nutzung der Lücken im Bestand an, da doppelt genutzter Kronenraum hierbei nicht erfasst wird. Die höhere beleuchtete Kronenmantelfläche im Mischbestand lässt darauf schließen, dass Lücken im Kronenraum besser genutzt werden als in den jeweiligen Reinbeständen. In Pretzsch et al., 2010 und Pretzsch et al., 2013 wurde gezeigt, dass die Mischbestände über längere Zeit eine signifikant höhere Kronenschirmfläche als benachbarte Reinbestände aufweisen und dies zu einer möglicherweise erhöhten Lichtinterzeption der Mischbestände führt. Diese erhöhte Lichtinterzeption wurde auch als möglicher Grund für einen Mehrzuwachs dieser Mischbestände genannt. Die erhöhte beleuchtete Kronenmantelfläche, welche durch die Anwendung des Kronenmodells in den Mischbeständen ermittelt wurde, 
bestätigt die Annahme der erhöhten Lichtinterzeption aus der Literatur.

Das erhöhte Kronenvolumen im Mischbestand könnte einerseits mit der besseren Lückenschließung zusammenhängen, da hierfür die Krone vergrößert werden muss. Andererseits bedeutet dies auch, dass die Lücken nicht auf Kosten von anderen Kronenteilen erschlossen wurden, sondern zusätzlich. Schröter et al. (2012) zeigt, dass sich die Buchenkronen stark von lokalen Konkurrenten weg richten. Dies könnte im Zusammenhang mit den vorgestellten Ergebnissen bedeuten, dass die Buche sich selbst ein größerer Konkurrent ist und deshalb im Reinbestand den Kronenraum unter nahe anderer Buchen nicht optimal ausnutzt. Dagegen ist der Konkurrenzdruck der Douglasien geringer, was es erlaubt in Mischung eher Lücken auszunutzen. Das Gesamtvolumen der Kronen bei der Buche hat sich erhöht. Dies könnte bedeuten, dass die Buche ihre Krone in Nachbarschaft zur Douglasie erhalten kann und zusätzlich Ressourcen für die Erschließung weiterer Lücken aufbringt, während sie im Buchen-Reinbestand lediglich eines von beiden schafft. Dass die Buche im Reinbestand primär auf den Erhalt des direkten Kronenraumes fokussiert ist, wird außerdem von den in Abbildung 22 vorgestellten Ergebnissen bestätigt, in welchen die Buche im Reinbestand rundere Kronen besitzt, als im Mischbestand.

\subsection{Waldbauliche Konsequenzen für die Behandlung von Buchen- Fichten und Buchen-Douglasien Mischbeständen}

Wie in Abschnitt $3.3 \mathrm{zu}$ erkennen ist, ist der positive Effekt für die Buche am größten, wenn die Beimischung zur Douglasie oder Fichte $25 \%$ beträgt, mit abnehmender Tendenz bei $50 \%$ und $75 \%$ Mischungsanteil (nach G/ha). Bei geringem Mischungsanteil kann die Buche am meisten von der veränderten Nachbarschaft durch die Koniferen profitieren. Da in dieser Untersuchung der Zuwachs der Bäume nicht erfasst wurde, wird der positive Zusammenhang von Kronengröße und Zuwachs (Wyckoff und Clark, 2005; Kramer, 1986) angenommen. Ähnliche Annahmen wurden in Pretzsch et al., 2010, 2013 getroffen, wo erhöhte Kronenschirm- 
flächen zu einer höheren Lichtinterzeption führten und letztendlich als Grund für einen Mehrzuwachs genannt wurden. Die Kronenvolumen im Mischbestand sind insgesamt größer als in den jeweiligen Reinbeständen (Abschnitt 3.3), was unter den getroffenen Annahmen zu einer erhöhten Produktivität des Mischbestandes führen müsste. Hierbei ist die Mischung mit Douglasie besonders positiv und die Mischung mit Fichte positiver als ein Buchen-Reinbestand. Die Produktivität wird allgemein als höher angesehen und als „Overyielding“ bezeichnet, wenn ein Mischbestand mehr produziert, als die durchschnittliche Produktivität der Reinbestände unter Berücksichtigung des Anteils der Arten im Bestand. „Transgressive Overyielding" liegt vor, wenn die Produktivität des Mischbestandes noch höher ist als die des produktivsten Reinbestandes (Forrester und Pretzsch, 2015; Ammer, 2019). Da das Kronenvolumen und die beleuchtete Kronenmantelfläche im Mischbestand größer sind als in beiden Reinbeständen, ist anzunehmen, dass es sich in den Mischbeständen mindestens um „Overyielding“" handelt. Die Frage nach „Transgressive Overyielding" könnte in Produktivitätsmessungen in hierauf folgenden Projekten mit Zuwachsdaten der Versuchsflächen genauer untersucht werden. Beispielsweise wurde in Pretzsch und Schütze (2009) „Transgressive Overyielding“ in Mischbeständen mit Buche und Fichte festgestellt. Auch Buche und Douglasie wiesen eine erhöhte Produktivität auf (Thurm und Pretzsch, 2016), was für die hier getroffenen Annahmen spricht. Aus ökonomischer Sicht könnte ein Mischbestand aus Buche-Douglasie somit einen einen Vorteil im Vergleich zu den Reinbeständen dieser Baumarten haben.

Für eine waldbauliche Betrachtung sollten allerdings auch die weiteren Funktionen des Waldes einbezogen werden. Alle Ökosystemfunktionen des Waldes sind auch gleichzeitig von ökonomischem Wert, auch wenn nicht alle Funktionen einen Markt haben (Pearce, 2001). Ein Waldökosystem erfüllt verschiedene Ökosystemfunktionen. Diese beinhalten unter anderem die produktive Funktion des Waldes (Holz), Schutz der biologischen Vielfalt, Boden- und Wasserschutz, oder sozioökonomische Funktionen (z.B. Sicherung der Jobs im Forstsektor). Man spricht 
hierbei von einer Multifunktionalität des Waldes (Röhrig et al., 2020), dessen Dimension jedoch immer wieder diskutiert wird (Puettmann et al., 2008; Ammer et al., 2009). Damit ein Waldbauer effizienter wird, muss er die Menge und den Wert des von ihm produzierten Holzes erhöhen und/oder die Betriebskosten senken (Moore et al., 2015). Allerdings muss in Anbetracht der verschiedenen Risiken auch die Stabilität des Waldökosystems mit einbezogen werden (Albert et al., 2017). Risikomodellierungen zeigen, dass Forstbetriebe, welche nur auf eine Baumart im Reinbestand setzen, nie die optimale Lösung sind (Friedrich et al., 2020). In dieser Arbeit wurde die Kronengröße und Struktur untersucht. Die beleuchtete Kronenmantelfläche sowie Kronengröße (Volumen, Kronenbreite) haben direkte Auswirkungen auf den Zuwachs der Bäume (Wyckoff und Clark, 2005; Kramer, 1986). Es kann somit angenommen werden, dass ein Mischbestand mit einem insgesamt größeren Kronenvolumen und beleuchteter Kronenmantelfläche auch einen höheren Zuwachs besitzt, als ein Reinbestand mit geringerem Kronenvolumen. Aus ökonomischen Gesichtspunkten ist zudem die Art wichtig, welche den Mehrzuwachs der Krone verursacht. Hierbei sind die Marktwerte der einzelnen Holzarten entscheidend. Wenn die Buche eine größere Krone hat, aber dies auf Kosten der Fichtenkronen geschieht, wobei Fichtenholz einen besseren Preis erzielt, dann ist es für einen forstwirtschaftlichen Betrieb aus ökonomischer Sicht nicht erforderlich, diesen Mischbestand zu etablieren. Natürlich gibt es neben der ökonomischen Funktion noch weitere Punkte für Mischbestände, wie Stabilität und Resilenz gegenüber Kalamitäten und Unwetter, Artenvielfallt und Diversität des eigenen Holzsortiments. So kann beispielsweise eine höhere Artenvielfalt in Mischbeständen zu einem stabileren Ökosystem beitragen und dadurch Schäden verringern (Speight et al., 1989). Einflüsse auf diese zusätzlichen Funktionen werden in den anderen Teilprojekten des GRK2300 untersucht. 


\subsection{Ausblick}

Möglichkeiten zur Verwendung der erfassten Kronenparameter und Strukturdaten können beispielsweise die Schätzung anderer, schwerer zu erfassenden Eigenschaften eines Waldbestandes sein. In Smith (1964); Kuiper und Coutts (1992) wurden bei Douglasien die Kronengrößen verwendet, um die Größe des Wurzelsystems zu schätzen. Auch Tubbs (1977) hat schon die Relation von Kronen und Wurzeln untersucht und kam zu dem Ergebnis, dass bei den untersuchten Bäumen (Ahorn) die maximalen Kronenradien mit den maximalen Wurzellängen um den Baum herum korrelieren. Ein anderes Beispiel ist die Verwendung von Baummerkmalen und Baumart, um Schäden von Kleinsäugern modellhaft zu schätzen Sullivan et al. (2001); Carey und Johnson (1995); Malcolm et al. (1995); Gill (1992). In Li et al. (2017) wurden die Kronengrößen als Schätzer für die Wachstumsrate der Verjüngung verwendet. Sterck et al. (2003) hat die Einflüsse verschiedener Kronenmerkmale auf das Wachstum und Überleben von Verjüngung untersucht und hat herausgefunden, dass die Kronenmerkmale relativ gute Schätzer für das zukünftige Wachstum und Überleben der Verjüngung sind. Daten über die Kronenstruktur können somit durchaus als Schätzer für weitere Untersuchungen oder Teilprojekte dieses Projektes verwendet werden.

Für die Zukunft dieses hier entwickelten Modellansatzes und der dafür benötigten fotogrammetrischen Vermessungsmethode wäre es interessant, ein größeres Spektrum an Baumarten, Mischungsformen sowie Altersspannen zu vermessen. Diese Weiterentwicklung würde das Kronenmodell wesentlich robuster und für eine größere Bandbreite an Waldbeständen einsetzbar machen. Die Methode auf dem jetzigen Stand dient nicht als Ersatz für terrestrisches Laserscanning (TLS). Es ist eine einfache und schnell durchzuführende Methode für die Erfassung von Kronenstrukturen. Sie bietet zwar nicht den Detailgrad des TLS an, jedoch ist auch die Auswertung schneller und die Aufnahme der Kronenfotos mit einem handelsüblichen Smartphone oder Tablet möglich. Wenn für die Parametrisierung des Kronenmodells und darauf folgende Strukturuntersuchungen die vertikale Kronen- 
größe von Interesse ist, können mit dieser Methode gezielt Daten dafür aufgenommen werden.

Die Fortsetzung des Teilprojektes 9 im Rahmen des Graduiertenkollegs wird sich unter anderem mit der Frage des Zuwachses beschäftigen, was ein hier noch nicht untersuchter, aber wichtiger Aspekt für den Vergleich von Rein- und Mischbestand ist. Cole und Lorimer (1994) haben das Baumwachstum von Esche, Ahorn und Linde aufgrund von deren Kronenvariablen geschätzt. Das Ergebnis zeigte einen großen Zusammenhang des Wachstums mit der beleuchteten Kronenoberfläche bei 21 verschiedenen Baumarten. Der Zusammenhang von Kronengröße und Zuwachs/Produktivität wurde auch in Stenberg et al. (1994); Wyckoff und Clark (2005); Kramer (1986) festgestellt. In der Fortsetzung des Projektes könnte diese Annahme dann mit Daten der Versuchsflächen verifiziert werden und die entwickelten Kronenmodelle könnten sich dadurch als noch relevanter für die Praxis darstellen. Wie in Abschnitt 3.3 gezeigt wurde, unterscheidet sich die beleuchtete Kronenmantelfläche auf den Versuchsflächen in Rein- und Mischbeständen, was folglich einen Einfluss auf das Wachstum haben sollte. Die Betrachtung dieses Zusammenhangs speziell in Bezug auf Rein- und Mischbestände wird wichtige Ergebnisse für das Gesamtprojekt liefern.

\section{$5 \quad$ Zusammenfassung}

In diesem Projekt wurden Kronenmerkmale untersucht, um deren Veränderung in Abhängigkeit der Nachbarschaft von Einzelbäumen zu erfassen und darauf aufbauend die Kronenstruktur und Raumnutzung in Rein- und Mischbeständen miteinander zu vergleichen. Für die Aufnahme der vertiakelen Kroneform wurde die Kronenfenstermethode verwendet und weiterentwickelt, um die Kronenform schnell und unkompliziert mit einem Tablet oder Smartphone zu erfassen. Aus diesen Daten wurden mit Hilfe von statistischen Modellen die Parameter einer Weibull-Funktion geschätzt, welche dann die Kronenform anhand der Nachbarschaft eines Einzelbaumes darstellt. Die Ergebnisse zeigen, dass die Unterschiede 
von Rein- und Mischbeständen vor allem der Anpassungsfähigkeit der Buchenkronen zuzuschreiben sind. Es wurde festgestellt, dass die Buchenkronen auf eine Mischung mit den Koniferen Fichte und Douglasie reagieren. In Mischung hat die Buche eine breitere Krone als im Reinbestand. Ihre Höhe der maximalen Kronenbreite befindet sich in Mischung mit Douglasie unterhalb der Douglasienkronen und zudem auch tiefer als im Buchenreinbestand. Des Weiteren weisen die $\mathrm{Bu}-$ chenkronen im Mischbestand eine ovalere und asymmetrischere Form auf. Bei der Fichte und Douglasie konnten ähnliche Effekte nicht gefunden werden. Die Douglasie hatte hingegen sogar eine rundere Kronenform in Mischung mit der Buche. Zusammenfassend kann gesagt werden, dass sich die Buche unter der höher wachsenden Douglasie ausbreiten kann und so möglicherweise den verlorenen oberen Kronenraum mit einer breiteren, aber tiefer liegenden Krone kompensiert.

Die Bestandesuntersuchungen mit Hilfe des hier konstruierten Kronenmodells bestätigen diese Aussage. Hierbei weisen die Mischbestände (simuliert, mit anteiliger Grundfläche der Reinbestände) der Buche mit beiden Koniferenarten eine höhere beleuchtete Kronenmantelfläche auf als die dazugehörigen Reinbestände. Die Buche konnte hier vor allem in geringer Mischung von 25\% Buche zu $75 \%$ Douglasie eine zum Reinbestand um Faktor 1,6 höhere beleuchtete Kronenmantelfläche erreichen, während die Douglasie in allen Mischungsvarianten keine Veränderung aufwies. Die Fichte hingegen hatte in Mischung mit der Buche auf Bestandessicht eine etwas geringere beleuchtete Kronenmantelfläche, je höher der Buchenanteil war. Insgesamt zeigen Mischbestände jedoch einen positiven Effekt bei der beleuchteten Bestandes-Kronenmantelfläche. Für das Kronenvolumen zeigt die Mischung von Buche mit Douglasie einen positiven Effekt, während bei der Mischung von Buche mit Fichte das erhöhte Kronenvolumen der Buche durch ein kleineres Kronenvolumen der Fichte zu einem Ausgleich führt. Eine Regressionsanalyse hat zudem gezeigt, dass mit dem Kronenmodell geschätzte Kronenradien bei Buche, Fichte und Douglasie vergleichsweise gut mit abgeloteten Kronenradien übereinstimmen. Folglich könnte das nachbarschaftssensitive Kronenmodell einen Vorteil 
für Wachstumssimulatoren bieten, in denen Kronenparameter eine wichtige Rolle spielen (beispielsweise TreeGrOSS) und welche bisher häufig mit mittleren Kronenbreiten arbeiten. 


\section{Danksagung}

Ich danke allen, die bei der Entstehung dieser Arbeit beteiligt waren.

Besonders bedanke ich mich bei Prof. Dr. Jürgen Nagel für die Betreuung der Arbeit, den vielen Gesprächen und Anregungen, welche mich auch in schwierigen Phasen motiviert haben.

PD Dr. Matthias Albert für wertvolle Verbesserungsvorschläge und Unterstützung.

Dr. Holger Sennhenn-Reulen für die Hilfe bei statistischen Fragen.

Prof. Dr. Christian Ammer für die Leitung des Gesamtprojektes und die Bereitschaft, die Arbeit zu begutachten.

Der Deutschen Forschungsgemeinschaft für die Förderung des Projektes - 316045089.

Dr. Serena Müller für die vielen organisatorischen Hilfeleistungen.

Meiner Büronachbarin Sonja Mollenhauer für die vielen schönen Gespräche.

Dem netten Kollegium an der NW-FVA, welche im Laufe der Jahre Daten zusammengetragen, bearbeitet und kontrolliert haben, die in dieser Arbeit Verwendung gefunden haben.

Meinen Kollegen aus dem Graduiertenkolleg für die schönen Treffen und den offenen Austausch.

Mihaela Manescu, die mir den Mut gegeben hat, mich auf eine Doktorandenstelle zu bewerben.

Roland Potthast für die Unterstützung bei der Datenaufnahme sowie den vielen Gesprächen auf unseren langen Rennradtouren.

Sebastian Nikelski für das Befeuern und Korrekturlesen.

Meinen Eltern für das Korrekturlesen, das Interesse und die moralische Unterstüzung.

Meiner Verlobten Isabell Scholle für die Motivation und ihren Glauben an mich. 


\section{Literatur}

Adler, Joseph (2010): $R$ in a nutshell: A desktop quick reference. Ö’Reilly Media, Inc.".

Albert, Matthias (1994): Erhebung von Strukturinformationen in einem BuchenEdellaubholz Mischbestand. Dissertation.

Albert, Matthias; Ralf-Volker Nagel; Robert S Nuske; Johannes Sutmöller und Hermann Spellmann (2017): Tree species selection in the face of drought risk uncertainty in forest planning. Forests, 8(10):363.

Ameztegui, Aitor; Lluís Coll; Raquel Benavides; Fernando Valladares und Alain Paquette (2012): Understory light predictions in mixed conifer mountain forests: Role of aspect-induced variation in crown geometry and openness. Forest Ecology and Management, 276:52-61.

Ammer, C; K Puettmann et al. (2009): The road ahead of forest stand management-single or multiple management objectives? Forstarchiv, 80(3):9096.

Ammer, Christian (2016): Unraveling the importance of inter-and intraspecific competition for the adaptation of forests to climate change. In: Progress in Botany Vol. 78, Springer, S. 345-367.

Ammer, Christian (2019): Diversity and forest productivity in a changing climate. New Phytologist, 221(1):50-66.

Bachmann, M (1996): Indizes zur Erfassung der Konkurrenz im Bergmischwaldeine Methodenstudie. Bericht Jahrestagung Sektion Ertragskunde im DVFFA: S, S. 249-265.

Barbeito, Ignacio; Mathieu Dassot; Dominik Bayer; Catherine Collet; Lars Drössler; Magnus Löf; Miren del Rio; Ricardo Ruiz-Peinado; David I Forrester; Andrés 
Bravo-Oviedo et al. (2017): Terrestrial laser scanning reveals differences in crown structure of Fagus sylvatica in mixed vs. pure European forests. Forest Ecology and Management, 405:381-390.

Bauhus, Juergen; PK Khanna und N Menden (2000): Aboveground and belowground interactions in mixed plantations of Eucalyptus globulus and Acacia mearnsii. Canadian Journal of Forest Research, 30(12):1886-1894.

Bauhus, Jürgen; Benno Pokorny; Peter J van der Meer; Peter J Kanowski und Markku Kanninen (2010): Ecosystem goods and services-the key for sustainable plantations. In: Ecosystem Goods and Services from Plantation Forests, Routledge, S. 221-243.

Bauhus, Jürgen und Joachim Schmerbeck (2010): Silvicultural options to enhance and use forest plantation biodiversity. In: Ecosystem goods and services from plantation forests, Routledge, S. 112-155.

Bauhus, Jürgen; Aaron P Van Winden und Adrienne B Nicotra (2004): Aboveground interactions and productivity in mixed-species plantations of Acacia mearnsii and Eucalyptus globulus. Canadian Journal of Forest Research, $34(3): 686-694$.

de Bello, Francesco; Sandra Lavorel; Sandra Díaz; Richard Harrington; Johannes HC Cornelissen; Richard D Bardgett; Matty P Berg; Pablo Cipriotti; Christian K Feld; Daniel Hering et al. (2010): Towards an assessment of multiple ecosystem processes and services via functional traits. Biodiversity and Conservation, 19(10):2873-2893.

Bengtsson, Jan (1998): Which species? What kind of diversity? Which ecosystem function? Some problems in studies of relations between biodiversity and ecosystem function. Applied Soil Ecology, 10(3):191-199.

Biber, Peter (1996): Konstruktion eines einzelbaumorientierten Wachstumssimu- 
lators für Fichten-Buchen-Mischbestände im Solling. Forschungszentrum Waldökosysteme.

Blume, Hans-Peter; Gerhard W Brümmer; Rainer Horn; Ellen Kandeler; Ingrid Kögel-Knabner; Ruben Kretzschmar; Karl Stahr; Berndt-Michael Wilke; Sören Thiele-Bruhn und Gerhard Welp (2010): Bodenverbreitung. In: Lehrbuch der Bodenkunde, Springer, S. 363-378.

Bolte, Andreas; Christian Ammer; Magnus Löf; Palle Madsen; Gert-Jan Nabuurs; Peter Schall; Peter Spathelf und Joachim Rock (2009): Adaptive forest management in central Europe: climate change impacts, strategies and integrative concept. Scandinavian Journal of Forest Research, 24(6):473-482.

Brolly, Gábor; Géza Király; Matti Lehtomäki und Xinlian Liang (2021): VoxelBased Automatic Tree Detection and Parameter Retrieval from Terrestrial Laser Scans for Plot-Wise Forest Inventory. Remote Sensing, 13(4):542.

Burschel, P und J Huss (2003): Grundriss des Waldbaus. 3., unveränderte Auflage. Eugen Ulmer GmbH \& Co., Stuttgart, Germany.

Carey, Andrew B und Murray L Johnson (1995): Small mammals in managed, naturally young, and old-growth forests. Ecological applications, 5(2):336-352.

Cole, William G und Craig G Lorimer (1994): Predicting tree growth from crown variables in managed northern hardwood stands. Forest Ecology and Management, 67(1-3):159-175.

Condés, Sonia und Hubert Sterba (2005): Derivation of compatible crown width equations for some important tree species of Spain. Forest Ecology and Management, 217(2-3):203-218.

Danson, F Mark; David Hetherington; Felix Morsdorf; Benjamin Koetz und Britta Allgower (2007): Forest canopy gap fraction from terrestrial laser scanning. IEEE Geoscience and remote sensing letters, 4(1):157-160. 
Díaz, Sandra; Joseph Fargione; F Stuart Chapin III und David Tilman (2006): Biodiversity loss threatens human well-being. PLoS Biol, 4(8):e277.

Döbbeler, H; M Albert; M Schmidt; J Nagel und J Schröder (2006): BWINPro. Programm zur Bestandesanalyse und Prognose. Handbuch zur Version, 6:125.

Ellenberg, Heinz und Christoph Leuschner (2010): Vegetation Mitteleuropas mit den Alpen: in ökologischer, dynamischer und historischer Sicht, Bd. 8104. Utb.

Engelmark, Ola; Kjell Sjöberg; Bengt Andersson; Ola Rosvall; Göran I Ågren; William L Baker; Pia Barklund; Christer Björkman; Don G Despain; Björn Elfving et al. (2001): Ecological effects and management aspects of an exotic tree species: the case of lodgepole pine in Sweden. Forest Ecology and Management, $141(1-2): 3-13$.

Enrico, RTG2300 (2021): EnriCo: Enrichment of European beech forests with conifers. Url: https://www.uni-goettingen.de/en/574362.html. Georg-AugustUniversitaet Goettingen, Goettingen, Germany.

Erickson, Heather E; Constance A Harrington und David D Marshall (2009): Tree growth at stand and individual scales in two dual-species mixture experiments in southern Washington State, USA. Canadian journal of forest research, 39(6):1119-1132.

Fahrmeir, Ludwig; Thomas Kneib und Stefan Lang (2007): Regression - Modelle, Methoden und Anwendungen. Springer.

Felbermeier, Bernhard und Reinhard Mosandl (2004): Fagus sylvatica. Enzyklopädie der Holzgewächse: Handbuch und Atlas der Dendrologie, S. 1-20.

Ferrarese, Jena; David Affleck; Carl Seielstad et al. (2015): Conifer crown profile models from terrestrial laser scanning. Silva Fenn, 49(1):1106. 
Forrester, David I (2014): The spatial and temporal dynamics of species interactions in mixed-species forests: from pattern to process. Forest Ecology and Management, 312:282-292.

Forrester, David I; Jürgen Bauhus; Annette L Cowie und Jerome K Vanclay (2006): Mixed-species plantations of Eucalyptus with nitrogen-fixing trees: a review. Forest Ecology and Management, 233(2-3):211-230.

Forrester, David I und Hans Pretzsch (2015): Tamm Review: On the strength of evidence when comparing ecosystem functions of mixtures with monocultures. Forest Ecology and Management, 356:41-53.

Forstplanungsamt, Niedersächsisches (2007): Forstliche Standortsaufnahme: Geländeökologischer Schätzrahmen Bergland. $Z u \quad f i n$ den in https://www.landesforsten.de/wp-content/uploads/2019/03/ standortskartierung-bergland-rahmenschema01-200\%.pdf [zitiert am 06.06.2021].

Forstplanungsamt, Niedersächsisches und LLUR (2009): Forstliche Standortsaufnahme: Geländeökologischer Schätzrahmen pleistozänes (diluviales) Tiefland. $\quad Z u$ finden in https://www.landesforsten.de/wpcontent/uploads/2019/03/standortskartierung-tiefland-rahmenschema062009.pdf [zitiert am 06.06.2021].

Frech, Annika; Christoph Leuschner; Marc Hagemeier und Dirk Hölscher (2003): Nachbarschaftsbezogene Analyse der Kronenraumbesetzung von Esche, Hainbuche und Winterlinde in einem artenreichen Laubmischwald (Nationalpark Hainich, Thüringen). Forstwissenschaftliches Centralblatt vereinigt mit Tharandter forstliches Jahrbuch, 122(1):22-35.

Friedrich, S; K Bödeker und T Knoke (2020): Der Wert und Preis von Mischwäldern. AFZ-Der Wald, (17):29-31.

Gadow, Klaus von (2003): Waldstruktur und Wachstum. Universitätsverlag Göttingen, Göttingen. 
Garnier, Eric und Marie-Laure Navas (2012): A trait-based approach to comparative functional plant ecology: concepts, methods and applications for agroecology. A review. Agronomy for Sustainable Development, 32(2):365-399.

Gill, RMA (1992): A review of damage by mammals in north temperate forests. 2. Small mammals. Forestry: An International Journal of Forest Research, $65(3): 281-308$.

Gill, Samantha J; Gregory S Biging und Edward C Murphy (2000): Modeling conifer tree crown radius and estimating canopy cover. Forest Ecology and Management, 126(3):405-416.

Guericke, Martin (2001): Untersuchungen zur Wuchsdynamik von Mischbeständen aus Buche und Europ. Lärche (Larix decidua, Mill.) als Grundlage für ein abstandsabhängiges Einzelbaumwachstumsmodell: Versuchsflächenanalyse, Modellparametrisierung und waldbauliche Konsequenzen für die Behandlung von Buchen-Lärchen-Mischbeständen im Südniedersächsischen Bergland. Cuvillier.

Hansen, Jan und Jürgen Nagel (2014): Waldwachstumskundliche Softwaresysteme auf Basis von TreeGrOSS-Anwendung und theoretische Grundlagen, Bd. 11. Niedersächsische Staats-und Universitätsbibliothek.

Hasenauer, Hubert (1997): Dimensional relationships of open-grown trees in Austria. Forest Ecology and Management, 96(3):197-206.

Hildebrandt, Patrick und Thomas Knoke (2009): Optimizing the shares of native tree species in forest plantations with biased financial parameters. Ecological Economics, 68(11):2825-2833.

Hillebrand, Helmut und Birte Matthiessen (2009): Biodiversity in a complex world: consolidation and progress in functional biodiversity research. Ecology letters, 12(12):1405-1419. 
Hobi, Martina L; Brigitte Commarmot und Harald Bugmann (2015): Pattern and process in the largest primeval beech forest of $\mathrm{E}$ urope ( $\mathrm{U}$ krainian $\mathrm{C}$ arpathians). Journal of Vegetation Science, 26(2):323-336.

Hooper, David U; FS Chapin Iii; John J Ewel; Andrew Hector; Pablo Inchausti; Sandra Lavorel; John Hartley Lawton; DM Lodge; Michel Loreau; Shahid Naeem et al. (2005): Effects of biodiversity on ecosystem functioning: a consensus of current knowledge. Ecological monographs, 75(1):3-35.

Hu, Jiafu; Sergio Angeli; Stefan Schuetz; Youqing Luo und Ann E Hajek (2009): Ecology and management of exotic and endemic Asian longhorned beetle Anoplophora glabripennis. Agricultural and Forest Entomology, 11(4):359-375.

Hu, Shuhua (2007): Akaike information criterion. Center for Research in Scientific Computation, 93 .

Hussein, Khalid A; Klaus von Gadow und Matthias Albert (2000): The Crown Window a simple device for measuring tree crowns. Forstwissenschaftliches Centralblatt vereinigt mit Tharandter forstliches Jahrbuch, 119(1-6):43-50.

IPCC, Climate Change (2007): The physical science basis.

Jones, Mark W und Richard Satherley (2000): Voxelisation: Modelling for Volume Graphics. In: VMV. S. 319-326.

Juchheim, Julia; Peter Annighöfer; Christian Ammer; Kim Calders; Pasi Raumonen und Dominik Seidel (2017): How management intensity and neighborhood composition affect the structure of beech (Fagus sylvatica L.) trees. Trees, $31(5): 1723-1735$.

Kanninen, Markku (2010): Plantation forests: global perspectives. In: Ecosystem goods and services from plantation forests, Routledge, S. 17-31.

Keenan, Rodney J (2015): Climate change impacts and adaptation in forest management: a review. Annals of Forest Science, 72(2):145-167. 
Kelty, Matthew J (1992): Comparative productivity of monocultures and mixedspecies stands. In: The ecology and silviculture of mixed-species forests, Springer, S. $125-141$.

Knoerzer, Dietrich (1999): Zur Naturverjüngung der Douglasie im Schwarzwald.

Kölling, C; T Knoke; P Schall und Ch Ammer (2009): Überlegungen zum Risiko des Fichtenanbaus in Deutschland vor dem Hintergrund des Klimawandels. Forstarchiv, 80(2):42-54.

Kramer, H (1986): Relation between crown parameters and volume increment of Picea abies stands damaged by environmental pollution. Scandinavian journal of forest research, 1(1-4):251-263.

Kramer, H und A Akça (1987): Leitfaden für Dendrometrie und Bestandesstruktur. Sauerlander's Verlag. Frankfurt am Main.

Kramer, Horst und Alparslan Akça (2008): Leitfaden zur Waldmesslehre. Sauerländer.

Kuiper, LC und MP Coutts (1992): Spatial disposition and extension of the structural root system of Douglas-fir. Forest ecology and management, 47(1-4):111125 .

Lamb, David; Peter D Erskine und John A Parrotta (2005): Restoration of degraded tropical forest landscapes. Science, 310(5754):1628-1632.

Lavorel, Sandra (2013): Plant functional effects on ecosystem services. Journal of Ecology, 101(1):4-8.

Lavorel, Sandra und Eric Garnier (2002): Predicting changes in community composition and ecosystem functioning from plant traits: revisiting the Holy Grail. Functional ecology, 16(5):545-556. 
Lebourgeois, François; N Gomez; P Pinto und Pierre Mérian (2013): Mixed stands reduce Abies alba tree-ring sensitivity to summer drought in the Vosges mountains, western Europe. Forest ecology and management, 303:61-71.

Lei, Pifeng; Michael Scherer-Lorenzen und Jürgen Bauhus (2012): Belowground facilitation and competition in young tree species mixtures. Forest Ecology and Management, 265:191-200.

Li, Ying; Wenzel Kröber; Helge Bruelheide; Werner Härdtle und Goddert Von Oheimb (2017): Crown and leaf traits as predictors of subtropical tree sapling growth rates. Journal of Plant Ecology, 10(1):136-145.

Lu, Huicui; Godefridus MJ Mohren; Jan den Ouden; Venceslas Goudiaby und Frank J Sterck (2016): Overyielding of temperate mixed forests occurs in evergreen-deciduous but not in deciduous-deciduous species mixtures over time in the Netherlands. Forest ecology and management, 376:321-332.

Luhmann, Thomas; Stuart Robson; Stephen Kyle und Ian Harley (2007): Close range photogrammetry. Wiley.

Lüpke, Bv (2009): Uberlegungen zu Baumartenwahl und Verjungungs-verfahren bei fortschreitender Klimaanderung in Deutschland. Forstarchiv, 80:67-75.

Malcolm, Jay R et al. (1995): Forest structure and the abundance and diversity of Neotropical small mammals. Forest canopies., S. 179-197.

Matthews, JD (1963): Factors affecting the production of seed by forest trees. In: Forestry abstracts. Bd. 24, S. 1-13.

Mehtätalo, Lauri (2006): Eliminating the effect of overlapping crowns from aerial inventory estimates. Canadian Journal of Forest Research, 36(7):1649-1660.

Metz, Jérôme; Peter Annighöfer; Peter Schall; Jorma Zimmermann; Tiemo Kahl; Ernst-Detlef Schulze und Christian Ammer (2016): Site-adapted admixed tree 
species reduce drought susceptibility of mature European beech. Global Change Biology, 22(2):903-920.

Millar, Constance I; Nathan L Stephenson und Scott L Stephens (2007): Climate change and forests of the future: managing in the face of uncertainty. Ecological applications, 17(8):2145-2151.

Mirzaei, Mehrdad; Jalal Aziz; Ali Mahdavi und Asma Mohammad Rad (2016): Modeling frequency distributions of tree height, diameter and crown area by six probability functions for open forests of Quercus persica in Iran. Journal of forestry research, 27(4):901-906.

Mitchell, Kenneth J (1975): Dynamics and simulated yieldof Douglas-fir. Forest Science, 21(suppl_1):a0001-z0001.

Mölder, Inga und Christoph Leuschner (2014): European beech grows better and is less drought sensitive in mixed than in pure stands: tree neighbourhood effects on radial increment. Trees, 28(3):777-792.

Moore, John; Peter Clinton et al. (2015): Enhancing the productivity of radiata pine forestry within environmental limits. N. Zeal. J. For, 60:35-41.

Mori, Shigeta und Akio Hagihara (1991): Crown profile of foliage area characterized with the Weibull distribution in a hinoki (Chamaecyparis obtusa) stand. Trees, 5(3):149-152.

Mutterer, J und W Rasband (2012): ImageJ Macro Language Programmer's Reference Guide v1. 46d. RSB Homepage, S. 1-45.

Nabuurs, Gert-Jan; A Pussinen; J Van Brusselen und MJ Schelhaas (2007): Future harvesting pressure on European forests. European Journal of Forest Research, 126(3):391-400.

Nadrowski, Karin; Christian Wirth und Michael Scherer-Lorenzen (2010): Is forest 
diversity driving ecosystem function and service? Current Opinion in Environmental Sustainability, 2(1-2):75-79.

Nagel, J; M Albert und M Schmidt (2002): Das waldbauliche Prognose-und Entscheidungsmodell BWINPro 6.1. Forst und Holz, 57(15/16):486-492.

Nagel, Jürgen (1999): Konzeptionelle Überlegungen zum schrittweisen Aufbau eines waldwachstumskundlichen Simulationssystems fur Nordwestdeutschland. Sauerlander Frankfurt am Main.

Nagel, Jürgen und Matthias Schmidt (2006): The silvicultural decision support system BWINPro. In: Sustainable Forest Management, Springer, S. 59-63.

Nichols, J Doland; Mila Bristow und Jerome K Vanclay (2006): Mixed-species plantations: prospects and challenges. Forest Ecology and Management, 233(23):383-390.

Oliver, Chadwick Dearing; Bruce C Larson und CD Oliver (1996): Forest stand dynamics. Wiley New York.

Otto, Hans-Jürgen (1991): Langfristige, ökologische Waldbauplanung für die niedersächsischen Landesforsten. Niedersächsisches Ministerium für Ernährung, Landwirtschaft und Forsten.

Oxbrough, Anne; Sergio García-Tejero; John Spence und John O’Halloran (2016): Can mixed stands of native and non-native tree species enhance diversity of epigaeic arthropods in plantation forests? Forest Ecology and Management, $367: 21-29$.

Ozanne, Claire MP; Dieter Anhuf; Sarah L Boulter; Michael Keller; Roger L Kitching; Christian Körner; Frederick C Meinzer; AW Mitchell; Tohru Nakashizuka; PL Silva Dias et al. (2003): Biodiversity meets the atmosphere: a global view of forest canopies. Science, 301(5630):183-186. 
Pearce, David W (2001): The economic value of forest ecosystems. Ecosystem health, 7(4):284-296.

Peters, Rob (1992): Ecology of beech forests in the northern hemisphere. Peters.

Petri, Hartmut (1966): Versuch einer standortgerechten, waldbaulichen und wirtschaftlichen Standraumregelung von Buchen-FichtenMischbeständen:(dargestellt für den nördlichen Teil von Rheinland-Pfalz). Ministerium für Landwirtschaft, Weinbau und Umweltschutz, Abt. Forsten.

Pinkard, E; M Battaglia; J Bruce; S Matthews; AN Callister; S Hetherington; I Last; S Mathieson; C Mitchell; C Mohammed et al. (2015): A history of forestry management responses to climatic variability and their current relevance for developing climate change adaptation strategies. Forestry: An International Journal of Forest Research, 88(2):155-171.

Pommerening, A und ST Murphy (2004): A review of the history, definitions and methods of continuous cover forestry with special attention to afforestation and restocking. Forestry, 77(1):27-44.

Pretzsch, H (1992a): Wuchsmodelle für Mischbestände als Herausforderung für die Waldwachstumsforschung. Forstwissenschaftliches Centralblatt vereinigt mit Tharandter forstliches Jahrbuch, 111(1):87-105.

Pretzsch, H und G Schütze (2005): Crown allometry and growing space efficiency of Norway spruce (Picea abies [L.] Karst.) and European beech (Fagus sylvatica L.) in pure and mixed stands. Plant Biology, 7(06):628-639.

Pretzsch, Hans (1992b): Konzeption und konstruktion von wuchsmodellen fur reinund mischbestande.

Pretzsch, Hans (2009): Forest dynamics, growth, and yield. In: Forest dynamics, growth and yield, Springer, S. 1-39.

Pretzsch, Hans (2019): Grundlagen der waldwachstumsforschung. Springer-Verlag. 
Pretzsch, Hans; Peter Biber und J Ďurskỳ (2002): The single tree-based stand simulator SILVA: construction, application and evaluation. Forest ecology and management, 162(1):3-21.

Pretzsch, Hans; Peter Biber; Enno Uhl; Jens Dahlhausen; Thomas Rötzer; Juan Caldentey; Takayoshi Koike; Tran Van Con; Aurélia Chavanne; Thomas Seifert et al. (2015): Crown size and growing space requirement of common tree species in urban centres, parks, and forests. Urban Forestry 63 Urban Greening, $14(3): 466-479$.

Pretzsch, Hans; Kamil Bielak; Joachim Block; Arkadiusz Bruchwald; Jochen Dieler; Hans-Peter Ehrhart; Ulrich Kohnle; Jürgen Nagel; Hermann Spellmann; Michał Zasada et al. (2013): Productivity of mixed versus pure stands of oak (Quercus petraea (M att.) L iebl. and Quercus robur L.) and European beech (Fagus sylvatica L.) along an ecological gradient. European Journal of Forest Research, 132(2):263-280.

Pretzsch, Hans; Joachim Block; Jochen Dieler; Phan Hoang Dong; Ulrich Kohnle; Jürgen Nagel; Hermann Spellmann und Andreas Zingg (2010): Comparison between the productivity of pure and mixed stands of Norway spruce and European beech along an ecological gradient. Annals of Forest Science, 67(7):712.

Pretzsch, Hans; Miren del Río; Gerhard Schütze; Ch Ammer; P Annighöfer; A Avdagic; I Barbeito; Kamil Bielak; Gediminas Brazaitis; L Coll et al. (2016): Mixing of Scots pine (Pinus sylvestris L.) and European beech (Fagus sylvatica L.) enhances structural heterogeneity, and the effect increases with water availability. Forest Ecology and Management, 373:149-166.

Pretzsch, Hans und Gerhard Schütze (2009): Transgressive overyielding in mixed compared with pure stands of Norway spruce and European beech in Central Europe: evidence on stand level and explanation on individual tree level. European Journal of Forest Research, 128(2):183-204. 
Prodan, Michail (1965): Holzmesslehre. Techn. Ber., Sauerlander.

Puettmann, Klaus J; K David Coates und Christian C Messier (2008): A critique of silviculture: managing for complexity. Island press.

Pya, Natalya und Simon N Wood (2015): Shape constrained additive models. Statistics and computing, 25(3):543-559.

Reyer, Christopher; Petra Lasch; Godefridus MJ Mohren und Frank J Sterck (2010): Inter-specific competition in mixed forests of Douglas-fir (Pseudotsuga menziesii) and common beech (Fagus sylvatica) under climate change-a modelbased analysis. Annals of Forest Science, 67(8):805.

Ripley, B (2012): RODBC: ODBC Database Access (2011). R package version $1.3-3$.

Röhle, H (1986): Vergleichende Untersuchungen zur Ermittlung der Genauigkeit bei der Ablotung von Kronenradien. Forstarchiv, 57:67-71.

Röhrig, Ernst; Norbert Bartsch und Burghard von Lüpke (2020): Waldbau auf ökologischer Grundlage. UTB GmbH.

Roloff, A und BM Grundmann (2008): Waldbaumarten und ihre Verwendung im Klimawandel. Archiv Forstwes. Landsch. ökol, 42:97-109.

Rothe, Andreas (1997): Einfluss des Baumartenanteils auf Durchwurzelelung, Wasserhaushalt, Stoffhaushaft und Zuwachsleistung eines Fichten Buchen Mischbestandes am Standort Höglwald.

Rothe, Andreas und Dan Binkley (2001): Nutritional interactions in mixed species forests: a synthesis. Canadian Journal of Forest Research, 31(11):1855-1870.

Sakamoto, Yosiyuki; Makio Ishiguro und Genshiro Kitagawa (1986): Akaike information criterion statistics. Dordrecht, The Netherlands: D. Reidel, 81(10.5555):26853. 
Schindelin, Johannes; Ignacio Arganda-Carreras; Erwin Frise; Verena Kaynig; Mark Longair; Tobias Pietzsch; Stephan Preibisch; Curtis Rueden; Stephan Saalfeld; Benjamin Schmid et al. (2012): Fiji: an open-source platform for biological-image analysis. Nature methods, 9(7):676-682.

Schmid, Max; Marco Pautasso und Ottmar Holdenrieder (2014): Ecological consequences of Douglas fir (Pseudotsuga menziesii) cultivation in Europe. European Journal of Forest Research, 133(1):13-29.

Schreuder, Hans T und Wayne T Swank (1974): Coniferous stands characterized with the Weibull distribution. Canadian Journal of Forest Research, 4(4):518523.

Schröder, J; D Gerold und H Röhle (2005): Modellierung der Kronenbreite in BWINPro-S für die Anwendung in strukturreichen Beständen. Sektion Ertragskunde Tagungsband 2005, S. 33.

Schröter, Matthias; Werner Härdtle und Goddert von Oheimb (2012): Crown plasticity and neighborhood interactions of European beech (Fagus sylvatica L.) in an old-growth forest. European Journal of Forest Research, 131(3):787-798.

Schuldt, Andreas und Michael Scherer-Lorenzen (2014): Non-native tree species (Pseudotsuga menziesii) strongly decreases predator biomass and abundance in mixed-species plantations of a tree diversity experiment. Forest ecology and management, 327:10-17.

Schulze, Ernst Detlef; Dominik Hessenmöller; Carolin Seele; Jana Wäldchen und Nikolas Von Lüpke (2010): Die Buche. Eine Kultur-und Wirtschaftsgeschichte. Biologie in unserer Zeit, 40(3):171-183.

Schume, Helmut; Georg Jost und Herbert Hager (2004): Soil water depletion and recharge patterns in mixed and pure forest stands of European beech and Norway spruce. Journal of Hydrology, 289(1-4):258-274. 
Senbeta, Feyera; Demel Teketay und Bert-Åke Näslund (2002): Native woody species regeneration in exotic tree plantations at Munessa-Shashemene Forest, southern Ethiopia. New forests, 24(2):131-145.

Smith, J Harry G (1964): Root spread can be estimated from crown width of Douglas fir, lodgepole pine, and other British Columbia tree species. The Forestry Chronicle, 40(4):456-473.

Speight, Martin R; David Wainhouse et al. (1989): Ecology and management of forest insects. Clarendon Press.

Spellmann, H; J Sutmöller und H Meesenburg (2007): Risikovorsorge im zeichen des klimawandels. Vorläufige Empfehlungen der NW-FVA am Beispiel des Fichtenanbaus. AFZ-DerWald, 23:1246-1249.

Sprauer, Susanne (2014): Modelle zur Abbildung asymmetrischer Kronenformen und zur Beschreibung der Zuwachsleistung für sechs Baumarten in Nordwestdeutschland.

Stenberg, Pauline; Timo Kuuluvainen; Seppo Kellomäki; Jennifer C Grace; Eric J Jokela und Henry L Gholz (1994): Crown structure, light interception and productivity of pine trees and stands. Ecological Bulletins, S. 20-34.

Sterck, F; M Martinéz-Ramos; G Dyer-Leal; J Rodríguez-Velazquez und L Poorter (2003): The consequences of crown traits for the growth and survival of tree saplings in a Mexican lowland rainforest. Functional Ecology, 17(2):194-200.

Sullivan, Thomas P; Druscilla S Sullivan und Pontus MF Lindgren (2001): Stand structure and small mammals in young lodgepole pine forest: 10-year results after thinning. Ecological Applications, 11(4):1151-1173.

Thomas, FM; R Bögelein; W Werner et al. (2015): Interaction between Douglas fir and European beech-investigations in pure and mixed stands. Forstarchiv, $86(4): 83-91$. 
Thurm, Eric A und Hans Pretzsch (2016): Improved productivity and modified tree morphology of mixed versus pure stands of European beech (Fagus sylvatica) and Douglas-fir (Pseudotsuga menziesii) with increasing precipitation and age. Annals of Forest Science, 73(4):1047-1061.

Thurm, Eric A; Enno Uhl und Hans Pretzsch (2016): Mixture reduces climate sensitivity of Douglas-fir stem growth. Forest Ecology and Management, 376:205220 .

Tubbs, Carl H (1977): Root-crown relations of young sugar maple and yellow birch. Research Note NC-225. St. Paul, MN: US Dept. of Agriculture, Forest Service, North Central Forest Experiment Station, 225.

Verheyen, Kris; Margot Vanhellemont; Thibout Stock und Martin Hermy (2007): Predicting patterns of invasion by black cherry (Prunus serotina Ehrh.) in Flanders (Belgium) and its impact on the forest understorey community. Diversity and Distributions, 13(5):487-497.

Violle, Cyrille; Marie-Laure Navas; Denis Vile; Elena Kazakou; Claire Fortunel; Irène Hummel und Eric Garnier (2007): Let the concept of trait be functional! Oikos, 116(5):882-892.

Vitali, Valentina; Ulf Büntgen und Jürgen Bauhus (2017): Silver fir and Douglas fir are more tolerant to extreme droughts than Norway spruce in south-western Germany. Global Change Biology, 23(12):5108-5119.

Vorreyer, Christian (1997): Untersuchungen von Buchen-und Douglasienkronen in Mischbeständen. Dissertation.

de Wall, Klaus (1995): Struktur und Leistung von Buchen-DouglasienMischbeständen im WuchsbezirkÜnterer Solling". Dissertation.

Weller, Andreas (2011): Prüfung der Anbaueignung von 38 autochthonen bzw. nichtautochthonen Douglasienherkünften (Pseudotsuga menziesii [Mirb.] Fran- 
co) in Bezug auf ihre Wuchsleistung und qualitative Entwicklung: Ergebnisse. Cuvillier Verlag.

Wensel, L; W Meerschaert; G Biging et al. (1987): Tree height and diameter growth models for northern California conifers. Hilgardia, 55(8):1-20.

Wermelinger, Beat (2004): Ecology and management of the spruce bark beetle Ips typographus-a review of recent research. Forest ecology and management, 202(1-3):67-82.

Werner, Earl E und Scott D Peacor (2003): A review of trait-mediated indirect interactions in ecological communities. Ecology, 84(5):1083-1100.

White, Timothy L (1987): Drought tolerance of southwestern Oregon Douglas-fir. Forest science, 33(2):283-293.

Williams, Laura J; Alain Paquette; Jeannine Cavender-Bares; Christian Messier und Peter B Reich (2017): Spatial complementarity in tree crowns explains overyielding in species mixtures. Nature Ecology $\&$ Evolution, 1(4):1-7.

Windreich, Guy; Nahum Kiryati und Gabriele Lohmann (2003): Voxel-based surface area estimation: from theory to practice. Pattern Recognition, 36(11):25312541.

Wood, S. N. (2011): Fast stable restricted maximum likelihood and marginal likelihood estimation of semiparametric generalized linear models. Journal of the Royal Statistical Society (B), 73(1):3-36.

Wood, Simon N (2001): mgcv: GAMs and generalized ridge regression for R. $R$ news, 1(2):20-25.

Wood, Simon N und Nicole H Augustin (2002): GAMs with integrated model selection using penalized regression splines and applications to environmental modelling. Ecological modelling, 157(2-3):157-177. 
Wu, Bin; Bailang Yu; Wenhui Yue; Song Shu; Wenqi Tan; Chunling Hu; Yan Huang; Jianping Wu und Hongxing Liu (2013): A voxel-based method for automated identification and morphological parameters estimation of individual street trees from mobile laser scanning data. Remote Sensing, 5(2):584-611.

Wyckoff, Peter H und James S Clark (2005): Tree growth prediction using size and exposed crown area. Canadian Journal of Forest Research, 35(1):13-20. 